\title{
Noncongruence subgroups and Maass waveforms
}

\author{
Fredrik Strömberg \\ School of Mathematical Sciences, The University of Nottingham, University Park, \\ Nottingham NG7 2RD, United Kingdom
}

\begin{abstract}
The main topic of the paper is spectral theory for noncongruence subgroups of the modular group. We have studied a selection of the main conjectures in the area: the Roelcke-Selberg and Phillips-Sarnak conjectures and Selberg's conjecture on exceptional eigenvalues. The first two concern the existence and nonexistence of an infinite discrete spectrum for certain types of Fuchsian groups and last states that there are no exceptional eigenvalues for congruence subgroups, or in other words, there is a specific spectral gap in the cuspidal spectrum.

Our main theoretical result states that if the corresponding surface has a reflectional symmetry which preserves the cusps then the Laplacian on this surface has an infinite number of "new" discrete eigenvalues. We define old and new spaces of Maass cusp forms for noncongruence subgroups in a way that provides a natural generalization of the usual definition from congruence subgroups and give a method for determining the structure of the old space algorithmically.

In addition to the theoretical result we also present computational data, including a table of subgroups of the modular group and eigenvalues of Maass forms for noncongruence subgroups. We also present, for the first time, numerical examples of both exceptional and (non-trivial) residual eigenvalues. To be able to (even heuristically) cerify lists of computed eigenvalues we also proved an explicit average Weyl's law in this setting.
\end{abstract}

Keywords: Noncongruence subgroups, Spectral theory;, Maass waveforms, Exceptional eigenvalues, Residual eigenvalues, Weyl's law

2010 MSC: 11F06, 11F72, 11F37, 11F32

\section{Introduction}

The aim of this paper is to study a selection of the most important open problems and conjectures in the $L^{2}$ spectral theory for Fuchsian groups and in particular congruence and noncongruence subgroups of the modular group. More precisely we are interested in the following three conjectures: the Roelcke-Selberg, Phillips-Sarnak and Selberg's eigenvalue conjecture. The first conjecture originally stated that a large class of Fuchsian

Email address: fredrik.stromberg@nottingham.ac.uk (Fredrik Strömberg) 
groups should have an infinite discrete spectrum. This conjecture was later revised to impose stronger restrictions on the groups for which it should apply. In a certain sense it is also opposed by the Phillips-Sarnak conjecture which asserts that "generic" Fuchsian groups should not have any, or at most a finite, discrete spectrum. Selberg eigenvalue conjecture, which asserts nonexistence of so-called exceptional eigenvalues for congruence subgroups.

Our approach to the Roelcke-Selberg conjecture is to prove the following theorem which gives a sufficient condition for the existence of an infinite discrete spectrum. This is also our main theoretical result.

Theorem 27. Let $G$ be a noncongruence subgroup of index at most 17 in the modular group. If $G$ has reflectional symmetry which preserve the cusp classes of $G$ then the space of new Maass cusp forms on $G$ is infinite-dimensional.

For details of what what is meant by a reflectional symmetry and a newspace for a noncongruence subgroups see Section 6.1. The proof uses a combination of theoretical results, mainly Theorems 23 and 26 and the classification of the old forms in Proposition 22 together with numerical computations. The Phillips-Sarnak and Selberg's eigenvalues conjectures, on the other hand, are much more difficult to approach theoretically. See Section 3 for more details. The approach to these conjectures has therefore instead been of a more exploratory nature. We tabulated a complete list of subgroups with index up to and including 17, together with a decomposition into conjugacy classes. For each conjugacy class we used algorithms analogous to those developed in [23, 65] to search for eigenvalues corresponding to Maass cusp forms. In all cases we only found eigenvalues for groups which either are congruence groups or cycloidal groups (i.e. have only one cusp), or have symmetries satisfying the hypothesis of the main theorem above. In all other cases we found no discrete cuspidal spectrum. In a few of these we did, however, find examples of residual spectrum, see Table 7.

Complete tables (databases) and all source code that has been used are available online at [67], or directly from the author.

In the next section we will present the relevant background and notation necessary for a reader without any previous knowledge of modular forms or Maass forms. This is then followed by a brief introduction to the spectral theory and Maass forms for general co-finite Fuchsian groups. This section also contains a more detailed description of the problems and conjectures we are interested in. Following this, we discuss how computed lists of eigenvalues can be certified and prove a new version of an explicit average Weyl's law. The fifth section is devoted to subgroups of the modular group and in particular to the correspondence between such groups and permutations. The final theoretical section includes a discussion of old and newform theory for noncongruence subgroups, including the action of symmetries. It also contains the steps involved in proving the main theorem as stated above, as well as a large number of explicitly worked out examples. The final sections contain a description of algorithms and methods related to verfying the correctness of data and a selection of numerical results.

\section{Background and Basic Definitions}

Let $\mathcal{H}=\{z=x+i y \mid y>0\}$ be the upper half-plane equipped with the hyperbolic line- and area-elements $d s=y^{-1}|d z|$ and $d \mu=y^{-2} d x d y$, respectively. The isometries of 
$\mathcal{H}$ are given by the group of real Möbius transformations $\mathrm{PGL}_{2}(\mathbb{R}) \simeq \mathrm{GL}_{2}(\mathbb{R}) / C$ where $C$ is the center of $\mathrm{GL}_{2}(\mathbb{R})$ and consists of diagonal matrices. The subgroup of orientation preserving isometries is $\mathrm{PSL}_{2}(\mathbb{R})$ and the quotient $\mathrm{PGL}_{2}(\mathbb{R}) / \mathrm{PSL}_{2}(\mathbb{R}) \simeq\{1, J\}$ where $J: z \mapsto-\bar{z}$ is the reflection in the imaginary axis. Following the usual convention we will use matrices in $\mathrm{GL}_{2}(\mathbb{R})$ to denote the elements of $\mathrm{PGL}_{2}(\mathbb{R})$ and let $\mathbb{1}_{2}$ be the identity matrix. Then $\gamma=\left(\begin{array}{ll}a & b \\ c & d\end{array}\right) \in \mathrm{PGL}_{2}(\mathbb{R})$ with $\operatorname{det} \gamma=a d-b c \neq 0$ acts on $\mathcal{H}$ by

$$
\gamma: z \mapsto \gamma z:= \begin{cases}\frac{a z+b}{c z+d} & \text { if } \operatorname{det} \gamma>0 \text { and } \\ \frac{a \bar{z}+b}{c \bar{z}+d} & \text { if } \operatorname{det} \gamma<0 .\end{cases}
$$

This action also extends in a natural way to the boundary $\partial \mathcal{H}=\mathbb{R} \cup\{\infty\}$ and if $f: \mathcal{H} \rightarrow \mathbb{C}$ is a function then $\gamma \in \mathrm{PGL}_{2}(\mathbb{R})$ acts on $f$ via the (weight 0 ) slash-action:

$$
\gamma: f \mapsto f \mid \gamma(z):=f(\gamma z) .
$$

An element $\gamma$ of $\mathrm{PSL}_{2}(\mathbb{R})$ is said to be elliptic, parabolic or hyperbolic if the absolute value of the trace of the associated matrix is smaller than, equal to or greater than 2 . Observe that $\gamma$ is elliptic, parabolic or hyperbolic if and only if it has one fixed point in $\mathcal{H}$, one (double) fixed point in $\partial \mathcal{H}$ or two (different) fixed points in $\partial \mathcal{H}$, respectively. Fixed points of parabolic elements are usually called cusps. All groups we consider in this paper has at least one cusp, $\infty$, which is fixed by the parabolic element $T^{w}: z \mapsto z+w$.

Let $\Gamma \leqslant \mathrm{PSL}_{2}(\mathbb{R})$ be a finitely generated Fuchsian group, that is, a discrete subgroup of $\mathrm{PSL}_{2}(\mathbb{R})$. It is common to identify the set of $\Gamma$-orbits, $\Gamma \backslash \mathcal{H}=\{\Gamma z: z \in \mathcal{H}\}$ with a fundamental domain for $\Gamma$ in $\mathcal{H}$. This set is denoted by $\mathcal{F}_{\Gamma}$ and we define it to be a (possibly closed) connected set in $\mathcal{H}$ with the property that $\Gamma \mathcal{F}_{\Gamma}=\cup_{\gamma \in \Gamma} \gamma\left(\mathcal{F}_{\Gamma}\right)=\mathcal{H}$ and if $\gamma, \gamma^{\prime} \in \Gamma$ then the intersection $\gamma \mathcal{F}_{\Gamma} \cap \gamma^{\prime} \mathcal{F}_{\Gamma}$ does not contain an interior point unless $\gamma=\gamma^{\prime}$. Note that all Fuchsian groups we consider are cofinite, or in other words, any choice of fundamental domain has finite hyperbolic area. They are also all of the first kind, meaning that their limit set is the boundary $\partial \mathcal{H}$. Geometrically the the space of orbits, $\Gamma \backslash \mathcal{H}$, can be viewed as an orbifold, that is, a (classical) Riemann surface with marked points and constant negative curvature -1 .

Our focus is on a particular type of Fuchsian groups, namely finite index subgroups of the modular group (note that sometimes the modular group is defined as $\mathrm{SL}_{2}(\mathbb{Z})$ )

$$
\mathrm{PSL}_{2}(\mathbb{Z})=\left\{\left(\begin{array}{ll}
a & b \\
c & d
\end{array}\right) \in \mathrm{PSL}_{2}(\mathbb{R}) \mid a d-b c=1, a, b, c, d \in \mathbb{Z}\right\} \simeq \mathrm{SL}_{2}(\mathbb{Z}) /\left\{ \pm \mathbb{1}_{2}\right\} .
$$

It is well-known that the modular group has the (standard) fundamental domain

$$
\mathcal{F}_{1}=\{z=x+i y \in \mathcal{H}:|z| \geq 1,|x| \leq 1 / 2\} .
$$

If $\Gamma$ is a subgroup of the modular group of finite index $h$ and $\mathrm{PSL}_{2}(\mathbb{Z})=\sqcup_{i=1}^{h} \Gamma V_{i}$ is a right coset decomposition then the set $\mathcal{F}_{\Gamma}=\cup_{i} V_{i} \mathcal{F}_{1}$ is a fundamental domain for $\Gamma$.

For a fixed positive integer $N$ we define a group homomorphism $\mathrm{PSL}_{2}(\mathbb{Z}) \rightarrow \mathrm{PSL}_{2}(\mathbb{Z} / N \mathbb{Z})$ given by entry-wise reduction modulo $N$. The kernel of this map is $\Gamma(N)$, the so-called principal congruence subgroup of level $N$. Any subgroup which contains a $\Gamma(N)$ is said to be a congruence subgroup and its level is the smallest such $N$. A subgroups which does not contain any $\Gamma(N)$ is called a noncongruence subgroup. 
Let $h$ be a positive integer and let $\mathrm{S}_{h}$ denote the group of permutations of the set $\{1,2, \ldots, h\}$. If $\pi, \sigma \in \mathrm{S}_{h}$ we define $\pi \sigma \in \mathrm{S}_{h}$ by $\pi \sigma(j)=\pi(\sigma(j))$ for $1 \leq j \leq h$. If $k$ is a positive integer a then $k$-cycle is a permutation $\pi \in \mathrm{S}_{h}$ with the property that there exist $k$ distinct elements $x_{1}, x_{2}, \ldots, x_{k} \in\{1,2, \ldots, h\}$ such that $\pi\left(x_{i}\right)=x_{i+1}, i=1, \ldots, k-1$ and $\pi\left(x_{k}\right)=x_{1}$. In so-called cycle notation we write $\pi=\left(x_{1} x_{2} \cdots x_{k}\right)$ with the implicit understanding that this representation of the cycle is in general not unique. Since we will often write down cycles for permutations it is useful to make the representations unique by choosing the "smallest" with respect to a lexicographical ordering, for instance we write (1 24$)$ instead of (241). The identity permutation will be denoted by $i d$.

\section{Maass waveforms and spectral theory}

The following section provides a brief overview of the relevant spectral theory for Fuchsian groups. For more details and proofs we refer to the textbooks by Iwaniec, [28], Venkov [71] and Hejhal [21].

Let $G$ be a co-finite but not co-compact Fuchsian group with equivalence classes of cusps represented by points $p_{1}, \ldots, p_{\nu_{\infty}} \in \mathbb{R} \cup\{i \infty\}$. Define the Hilbert space $L^{2}(G \backslash \mathcal{H})$ as the complex vector space of measurable functions $f: \mathcal{H} \rightarrow \mathbb{C}$ such that $f(\gamma z)=f(z)$ for all $\gamma \in G, z \in \mathcal{H}$ and $\|f\|^{2}=\langle f, f\rangle<\infty$, together with the Petersson inner product

$$
\langle f, g\rangle=\int_{G \backslash \mathcal{H}} f(z) \overline{g(z)} d \mu(z) .
$$

Here $G \backslash \mathcal{H}$ denotes any measurable fundamental domain of $G$ and the inetegral is independent of the choice. The Laplace-Beltrami operator on the upper half-plane with respect to the hyperbolic metric is given by

$$
\Delta=-y^{2}\left(\frac{\partial^{2}}{\partial x^{2}}+\frac{\partial^{2}}{\partial y^{2}}\right)
$$

and it is easy to verify that it commutes with the action of $\mathrm{PSL}_{2}(\mathbb{R})$. It is well-known that $\Delta$ has a unique extension to a non-negative self-adjoint operator on $L^{2}(G \backslash \mathcal{H})$ and we define a Maass waveform for $G$ to be a function $f \in C^{2}(\mathcal{H}) \cap L^{2}(G \backslash \mathcal{H})$ which satisfies:

$$
\Delta f=\lambda f \text { for some } \lambda \geq 0 \text { and }\|f\|^{2}>0 .
$$

If, additionally, $f(z) \rightarrow 0$ as $z$ approaches any parabolic fixed point of $G$, we say that $f$ is a Maass cusp form. Since $\Delta$ is a non-negative elliptic differential operator it follows from elliptic regularity that every Maass waveform is in fact real analytic and that we can write the eigenvalue as $\lambda=\frac{1}{4}+R^{2}$ where the spectral parameter, $R$, belongs to the set $\mathbb{R} \cup i\left[0, \frac{1}{2}\right]$. Another useful and common convention is to write $\lambda=s(1-s)$ where $s=1 / 2+i R$. For simplicity we will write $\mathcal{M}(G, R)$ and $\mathcal{M}^{0}(G, R)$ for the vector spaces of Maass waveforms and cusp forms on $G$ with eigenvalue $\lambda=1 / 4+R^{2}$. The direct sum over all eigenvalues for these spaces are denoted by $\mathcal{M}(G)$ and $\mathcal{M}^{0}(G)$.

For every cusp $p_{j}$ of $G$ we choose a cusp-normalizing map $\mathcal{N}_{j} \in \mathrm{PSL}_{2}(\mathbb{R})$ such that $\mathcal{N}_{j}(i \infty)=p_{j}$ and $\mathcal{N}_{j}^{-1} S_{j} \mathcal{N}_{j}=T$, where $S_{j}$ is a generator of $G_{p_{j}}$, the stabilizer of $p_{j}$ in $G$, and $T z=z+1$. The choice of $\mathcal{N}_{j}$ is unique up to powers of $T$. Observe that this choice implies that we are essentially replacing the cusp $p_{j}$, having width $h_{j}$ with 
the cusp $\infty$, having width 1 . This normalization makes it easier to work with Fourier expansions numerically in a uniform way. If $f \in \mathcal{M}^{0}(G, R)$ then the function $f_{j}=f \mid \mathcal{N}_{j}$ is periodic with period 1 , and by using separation of variables

$$
f_{j}(z)=\sum_{n \neq 0} c_{j}(n) \kappa_{n}(y ; i R) e(n x),
$$

where $z=x+i y \in \mathcal{H}, \kappa_{n}(y ; s)=\sqrt{y} K_{s}(2 \pi|n| y)$ with $K_{s}(y)$ the $K$-Bessel function, and $e(x)=e^{2 \pi i x}$. The constant term, $c_{j}(0)$, vanishes since $f$ is a cusp form. We say that this is the Fourier expansion of $f$ at the cusp $p_{j}$ and that the complex numbers $c_{j}(n)$ are the Fourier coefficients. It is useful to remember that the $K$-Bessel function $K_{i R}(y)$ with imaginary argument is real-valued.

Although it is not known in general if there exist any Maass cusp forms for a given group, it is always possible to construct a non-cuspidal eigenfunction of the Laplacian. The non-holomorphic Eisenstein series associated with the cusp $p_{i}$ of $G$ is defined as

$$
E_{i}(z ; s)=\sum_{\gamma \in G_{p_{i}} \backslash G} \Im\left(\mathcal{N}_{i}^{-1} \gamma z\right)^{-s},
$$

where $z \in \mathcal{H}$ and $s \in \mathbb{C}$ with $\Re(s)>1$. It can be shown that $E_{i}(z ; s)$ is an eigenfunction of $\Delta$ with eigenvalue $s(1-s)$ and that $s \mapsto E_{i}(z ; s)$ has a meromorphic continuation to the entire complex plane. Moreover, it has a Fourier expansion at the cusp $p_{j}$ of the form

$$
\begin{aligned}
E_{i j}(z ; s) & =c_{i j}(0 ; y ; s)+\sum_{n \neq 0} c_{i j}(n ; s) \kappa_{n}(y ; s-1 / 2) e(n x), \\
c_{i j}(0 ; y ; s) & =\delta_{i j} y^{s}+\varphi_{i j}(s) y^{1-s},
\end{aligned}
$$

where $\delta_{i j}$ is the usual Kronecker delta function and the "constant term", $c_{i j}(0 ; y ; s)$, can be interpreted in terms of scattering states entering from the cusp $p_{i}$ and exiting through the cusp $p_{j}$. The $\nu_{\infty} \times \nu_{\infty}$ matrix $\Phi(s)$ with entries $\varphi_{i j}(s)$ is therefore often called the scattering matrix and its determinant, $\varphi(s)$, the scattering determinant.

The main result in the spectral theory for $G$ (or $G \backslash \mathcal{H}$ ) is that the Hilbert space $L^{2}(G \backslash \mathcal{H})$ has a spectral decomposition

$$
L^{2}(G \backslash \mathcal{H})=\mathbb{C} \oplus \mathcal{M}^{0}(G) \oplus \mathcal{M}^{\mathrm{res}}(G) \oplus \mathcal{E}(G)
$$

where $\mathbb{C}$ corresponds to the constant function, $\mathcal{M}^{0}(G)$ and $\mathcal{M}^{\text {res }}(G)$ correspond to the discrete spectrum and are spanned by Maass cusp forms and non-constant residues of Eisenstein series, respectively, and $\mathcal{E}(G)$ is the continuous part, which is spanned by eigenpackets associated with the Eisenstein series.

By the non-negativity of $\Delta$ it follows that all non-constant discrete eigenfunctions have eigenvalues in the positive real axis and it can be shown that the residues of Eisenstein series actually all have eigenvalues in in the interval $[0,1 / 4$ ) (see e.g. [21, pp. 371-3]).

There are two fundamental questions in spectral theory in general, and which interest us here in particular:

- is the discrete spectrum infinite?

- is there a spectral gap? more precisely, is there a lower bound for the smallest non-zero discrete eigenvalue? 
Observe that if $f$ is a Maass waveform or cusp form for $G$ then it is also a Maass waveform or cusp form for all subgroups of $G$. It is therefore sensible to reformulate the above questions in terms of "new" spectra. For a formal definition of what we mean by old and new Maass cusp forms for noncongruence subgroups see Section 6.1.

\subsection{Existence of discrete spectrum}

Since we assumed that the group $G$ has cusps it is clear that the continuous spectrum exists simply by construction of the Eisenstein series. In contrast, the existence or nonexistence of a discrete spectrum is in general a very difficult problem. Let $N(T)$ be the counting function for the cuspidal discrete spectrum embedded inside $[1 / 4, \infty)$, i.e.

$$
N(T)=\mid\left\{\lambda=1 / 4+R^{2} \mid 0 \leq R \leq T \text { and } \Delta f=\lambda f \text { for some } f \in \mathcal{M}^{0}(G, R)\right\} \mid .
$$

As usual all eigenvalues are counted according to multiplicity and $N(T)$ is defined by right-continuity if $1 / 4+T^{2}$ is an eigenvalue. Let $M(T)$ be the counting function for the winding number of the scattering determinant $\varphi$ :

$$
M(T)=\frac{1}{4 \pi} \int_{-T}^{T} \frac{-\varphi^{\prime}}{\varphi}\left(\frac{1}{2}+i t\right) d t .
$$

By using the Selberg trace formula it can be shown that the two functions $N(T)$ and $M(T)$ satisfy an asymptotic relation, a Weyl's law, of the form

$$
N(T)+M(T)=\frac{\operatorname{vol}(G \backslash \mathcal{H})}{4 \pi} T^{2}+O(T \ln T) \quad \text { as } \quad T \rightarrow \infty
$$

and if $N(T) \sim \frac{1}{4 \pi} \operatorname{vol}(G \backslash \mathcal{H}) T^{2}$ then the group $G$ is said to be essentially cuspidal. See for instance [21, pp. 207-9] for the general case, or a more detailed version for the modular group in [21, Ch. 11]. It is therefore clear that to show that there exists an infinite number of discrete eigenvalues it is sufficient to find a good asymptotic bound for the growth of $M(T)$. It is known that for the modular group we have

$$
\varphi(s)=\frac{\Lambda(2-2 s)}{\Lambda(2 s)},
$$

where $\Lambda(s)=\pi^{-s / 2} \Gamma(s / 2) \zeta(s)$ is the completed Riemann zeta function. By using Stirling's formula and bounds for $\zeta$ on the line $\Re s=1$ it can be shown that $M(T)=$ $O(T \ln T)$ and hence that the modular group is essentially cuspidal and in particular has infinitely many discrete cuspidal eigenvalues. It turns out that a similar expression for the scattering determinant $\varphi(s)$ holds for congruence subgroups of $\mathrm{PSL}_{2}(\mathbb{Z})$, see e.g. $[21$, Ch 11.4-5] and [25], where the Riemann zeta function is replaced by (products of) more general Dirichlet $L$-functions and the case of $\Gamma_{0}(N), \Gamma_{1}(N)$ and $\Gamma(N)$ are treated in detail. It is easy to see that these $L$-functions still satisfy a similar bound and this can be used to show that these groups also have an infinite discrete spectrum (in addition to that associated with any supergroups). For a detailed proof see e.g. [51].

For a more general co-finite non co-compact Fuchsian group it turns out that the function $\varphi(s)$ can indeed also be expressed in terms of a Dirichlet series with an analytic continuation and a functional equation. This will in general, however, not be an 
L-function. In particular, it will not possess an Euler product and is not known (or expected) to satisfy bounds similar to those of $\zeta$ and may even have a large number of poles in the region to the left of the critical line $\Re s=1 / 2$. It can indeed be shown that $M(T)$ is roughly equal to the number of such poles up to height $T$, up to an error of magnitude $O(T)$ (cf. e.g. [28, Sect. 11.1].)

It is therefore currently believed that the abundance of discrete cuspidal eigenvalues exhibited by the modular group and its congruence subgroups is an exception from the norm, and due to arithmetic, rather than geometric properties of the surface. This question was studied by Phillips and Sarnak in a sequence of papers using deformation theory (cf. e.g. $[46-48,53])$ and led them to the following conjecture

Conjecture 1 (Phillips-Sarnak). If $G$ is a nonarithmetic fuchsian group of the first kind which is co-finite but not co-compact then $G$ is not essentially cuspidal.

In [46] they in fact made an even stronger remark that they believe that there should only be a finite number of discrete eigenvalues in this case. This statement is certainly false, as evidenced by the co-finite non-arithmetic Hecke triangle groups, all of which are not essentially cuspidal but nevertheless possess an infinite number of odd discrete eigenvalues. However, replacing "nonarithmetic" by "generic" (in Teichmüller sense) the statement seems to be consistent with all known theoretical and numerical experiments. Cf. e.g. Avelin [4] and Farmer-Lemurell [16].

Although finite index subgroups of the modular group are by no means generic, the numerical investigations reported on in the current paper has led to the belief that discrete cuspidal eigenvalues do not appear by accident for these groups. More precisely, the computations indicate that if $G$ is a finite index subgroup of the modular group then the non-exceptional (see below) discrete spectrum consists of an "old" part, associated with supergroups $G^{\prime}$ with $G<G^{\prime} \leqslant \mathrm{PSL}_{2}(\mathbb{Z})$, and the orthogonal complement, the "new" part is only infinite if $G$ is a congruence group, a cycloidal group (i.e. has only one cusp), or has a symmetry in form of a reflection in a geodesic. We are therefore confident in the following conjecture.

Conjecture 2. If $G \leqslant \mathrm{PSL}_{2}(\mathbb{Z})$ is a finite index noncongruence subgroup with more than one cusp then the new discrete spectrum of $G$ is finite unless $\Gamma$ has a symmetry given by a reflection which preserves cusps, in which case it is infinite and all associated Maass cusp forms are odd with respect to this symmetry.

Note that we found residual eigenvalues on groups without symmetry (see Table 7) but there will always be at most a finite number of these. The precise description of the symmetries are given in Section 6.3. There is of course nothing to prevent an individual Teichmüller curve, as investigated in [16], which preserves a Maass form under deformation, to intersect more than one subgroups of the modular group and thereby creating a "sporadic" Maass form, but the likelihood of this seems small.

\subsection{Small eigenvalues}

By small eigenvalues we mean the (non-zero) discrete eigenvalues inside the interval $[0,1 / 4)$, and by exceptional eigenvalues we mean small eigenvalues associated to Maass cusp forms. In contrast to the residual eigenvalues, which are all contained in $[0,1 / 4)$ 
and therefore small, the existence or non-existence of exceptional eigenvalues are more mysterious.

There are many, more or less elementary, methods available to show that the first non-zero discrete eigenvalue of the full modular group is larger than $1 / 4$ (in particular there is no residual spectrum) and hence that there are no small eigenvalues in this case. See [21, p.511, pp. 581-90] for an overview of some of the these methods. Based on this observation and the fact that congruence groups also do not have residual spectrum Selberg made the following conjecture:

Conjecture 3 (Selberg). There are no small eigenvalues for congruence subgroups of the modular group.

This conjecture can be understood as the Archimedean analogue of the general Ramanujan conjectures. For more information about this see the excellent overview in [8] and also [54]. Selberg's conjecture has been verified in individual cases by both geometric methods [26] (up to level 18) and trace formula methods [9] (squarefree levels < 857). Partial results have also been obtained in various directions. Selberg showed that the smallest discrete eigenvalue for a congruence subgroup satisfies $\lambda_{1} \geq 3 / 16$ and this lower bound has since been improved upon by many authors, the best published result is by Kim and Sarnak, $\lambda_{1} \geq 975 / 4096$ [30]. For an alternative approach see Risager [52]. In contrast, if we consider non-congruence subgroups then it is possible to construct subgroups with small eigenvalues. Selberg [56] gave a method to construct groups with residual eigenvalues arbitrarily close to 0 . It is also possible to give a purely geometric sufficient condition for the existence of small eigenvalues. Let $G$ be a co-finite Fuchsian group of the first kind with co-volume $\mu(G \backslash \mathcal{H})$ and genus $g$. If $\mu(G \backslash \mathcal{H}) \geq 32 \pi(g+1)$ then a theorem of Zograf [73] implies that $G$ has small eigenvalues. To find examples of exceptional eigenvalues we first observe that if $G$ is a cycloidal subgroup of the modular group then it has no residual eigenvalues since the only residues of Eisenstein series are constants. This follows immediately from the fact that the scattering matrix of such a group is equal to that of the modular group, up to a simple explicit factor. See e.g. [70].

Thus, if we find a cycloidal subgroup of genus 0 and index 96 then $\mu(G \backslash \mathcal{H})=96 \cdot \pi / 3=$ $32 \pi$ and hence it must have an exceptional eigenvalue. Although a complete list of such subgroups (and in particular a separation into conjugacy classes) is currently beyond reach with the computational methods described in Section 5.3 it is easy to use these methods to produce individual examples of groups with a given signature. In this manner we produced a list of cycloidal groups with genus 0 and index 96 and the corresponding exceptional eigenvalues we found are given in Table 9. After these initial examples, which showed that our algorithms also work for exceptional eigenvalues, we set out to investigate whether the bound by Zograf is sharp or not. In other words, we wanted to locate exceptional eigenvalues on subgroups of smaller index.

Since the permutations which specify the groups of index 96 and 48 are very large we only give the signatures of these groups in Tables 10 and 9 . The complete details necessary to reconstruct the groups can be found at [67].

\section{Counting Eigenvalues and the Average Weyl's Law}

As mentioned in the previous section the basic version of Weyl's law (6) is sufficient to establish the existence of discrete spectrum in case the continuous term can be estimated 
efficiently. To investigate the finer properties of the distribution of eigenvalues we need a refinement analogous to the following version given in [21, Thm. 2.28]. If $G$ is a co-finite Fuchsian group with $e_{\infty}$ cusps and $T \geq 1$ then

$$
N(T)+M(T)=\frac{\operatorname{vol}(G \backslash \mathcal{H})}{12} T^{2}-\frac{e_{\infty}}{\pi} T \log (2 T / e)+c+S(T)+O(1 / T),
$$

where $N(T)$ and $M(T)$ are as in (4) and (5), $c$ is a constant and $S(T)$ is a function satisfying $S(T)=O(T)$ and $\int_{1}^{T} S(t) d t=O(T)$. There are several possibilities to improve upon this formula. For specific groups, for instance groups with one cusp, or congruence subgroups, it is possible to give an explicit expression for $\varphi(s)$ and hence also $M(T)$. In particular one can easily obtain that $M(T)=O(T \ln T)$ in these cases.

A further improvement is obtained by evaluating the constant term and the implied constant in the big-Oh term as explicitly as possible. The currently best known result in this direction is given by Jorgenson, Smajlović and Then [29] who, for the particular case of a so-called moonshine group, $\Gamma_{0}(N)^{+}$, with square-free $N$ show that

$$
N(T)=\frac{\operatorname{vol}\left(\Gamma_{0}(N)^{+} \backslash \mathcal{H}\right)}{4 \pi} T^{2}-\frac{2}{\pi} T \ln \left(\frac{T}{e \sqrt{\pi /(2 N)}}\right)+c_{1}(T)+c_{2}+G_{N}(T)+S_{N}(T),
$$

where $c_{1}(T)$ and $c_{2}$ are completely explicit. The (non-explicit) error terms satisfy $\left|G_{N}(T)\right|<$ $c_{3} / T$ for all $T>1$ with an explicit constant $c_{3}$ and $\int_{0}^{T} S_{N}(t) d t=O\left(T / \ln ^{2} T\right)$ as $T \rightarrow \infty$. The group $\Gamma_{0}(N)^{+}$has one cusp, genus 1 and contains the congruence subgroup $\Gamma_{0}(N)$. A special case is $\Gamma_{0}(1)^{+}$which is just the modular group and in this case their result is

$$
N(T)=\frac{1}{12} T^{2}-\frac{2}{\pi} T \ln \left(\frac{T}{e \sqrt{\pi / 2}}\right)-\frac{131}{144}+G_{1}(T)+S_{1}(T)
$$

where $\left|G_{1}(T)\right|<1 / T$ and $S_{1}(T)$ is as above. Compare [21, p. 511].

To apply Turing's method to the Selberg zeta function, or in other words, to determine if a computed list of discrete eigenvalues is complete, it is necessary to obtain yet another improvement over (7). In particular, it is necessary to have explicit estimates for all terms. Currently the only concrete application of Turing's method in this setting is given by Booker and Platt [10] for the modular group. They show that if (8) is written

$$
N(T)=\bar{N}(T)+S(T)
$$

where $S(T)=G_{1}(T)+S_{1}(T)$ then

$$
\frac{1}{T} \int_{0}^{T} S(t) d t \leq E(T)=\left(1+\frac{6.59125}{\ln T}\right)\left(\frac{\pi}{12 \ln T}\right)^{2} \quad \text { for all } T>1 .
$$

The idea behind Turing's method is to first obtain an upper bound

$$
\int_{0}^{T} N(t) d t=\int_{0}^{T} \bar{N}(t)+S(t) d t<X
$$

and then compare this with the integral of a minorant, $N_{\exp }(t) \leq N(t)$, given as an experimental counting function from a set of computed eigenvalues. If we assume that

$$
\int_{0}^{T} N_{\exp }(t) d t>X_{0}>X
$$


and that there is a single eigenvalue, $r$, missing in the computed set up to $T$ then

$$
\int_{0}^{T} N(t) d t=\int_{0}^{T} N_{\exp }(t) d t+(T-r)>X_{0}+(T-r) .
$$

It follows that in this case there can be no eigenvalue missing with $r<T+X_{0}-X$, hence the list up to $T+X_{0}-X$ must be complete. As a final step to transform this argument into a rigorous proof Booker and Platt uses rigorous quadrature and interval arithmetic to evaluate the integrals and estimates.

Unfortunately it is problematic to apply either of these methods directly in our setting of a general subgroup of the modular group. First of all, the scattering determinant is not given as an explicit quotient of $L$-functions and we are therefore unable to obtain an efficient estimate for $M(T)$. Note that this is not only due to lack of information: from experimental data it seems that the function $|M(T)|$ in some cases grow as rapidly as the "main term" in Weyl's law. We therefore need to keep this term as an experimentally computed quantity (see Section 10). In addition, to obtain bounds for $S(T)$ similar to those in (9) it is necessary to control the hyperbolic terms in the Selberg trace formula, and this seems intractable for general subgroups.

To present the main result of this section in a clear and precise manner we need to distinguish between different types of discrete eigenvalues. Therefore, let $\Sigma_{\text {res }}$ be the set of residual eigenvalues (note that we include the eigenvalue 0 in this set), $\Sigma_{\text {exc }}$ the set of exceptional cuspidal eigenvalues, i.e. eigenvalues $\lambda<1 / 4$ with corresponding eigenfunction a Maass cusp form.

We then need to refine the counting functions as well. In addition to $N(T)$ and $M(T)$, which are still given by (4) and (5) we let $N_{\text {res }}$ and $N_{\text {exc }}$ be the number of residual and exceptional eigenvalues, respectively (all counted according to multiplicity).

The main result for this section, which we will use to provide a heuristic argument to support claims that a computed list of eigenvalues is complete, is the following:

Theorem 4. Let $G$ be a finite index subgroup of the modular group with signature $\left(h ; g, e_{\infty}, e_{2}, e_{3}\right)$ and let $N_{r e s}, N_{e x c}, N(T)$ and $M(T)$ be as above. Then, for $T \geq 1$ :

$$
N(T)+N_{\text {res }}+N_{\text {exc }}+M(T)=\frac{h}{12} T^{2}-\frac{e_{\infty}}{\pi} T \log (2 T / e)+c_{G}+g(T)+S(T),
$$

where

$$
c_{G}=-h \frac{1}{144}+e_{2} \frac{1}{8}+e_{3} \frac{2}{9}-e_{\infty} \frac{1}{4}+\frac{C}{2}
$$

with $C=\frac{1}{2} \operatorname{Tr}(\mathbb{1}-\Phi(1 / 2))$ and $g(T)$ and $S(T)$ are functions satisfying

$$
|g(T)| \leq\left(h \frac{1}{8707}+e_{2} \frac{1}{139}+2 e_{3} \frac{1}{67}+e_{\infty} \frac{26}{75}\right) \cdot \frac{1}{T}
$$

for all $T \geq 1$,

$$
S(T)=O\left(\frac{T}{\ln T}\right) \quad \text { and } \quad\langle S(T)\rangle:=\frac{1}{T} \int_{0}^{T} S(t) d t=O\left(\frac{1}{\ln ^{2} T}\right) \quad \text { as } \quad T \rightarrow \infty .
$$


Proof. The key steps of the proof are similar to those in [29], building on the proof of [21, Thm. 2.28, p. 466]. The idea is to express each term as explicitly as possible and then use the explicit formula for the scattering determinant (see also [21, pp. 508-511]).

The main difference with our result is that we need to allow for an arbitrary number of cusps, and since we only have elliptic fixed points of order 2 and 3 certain terms can be made more explicit. It is also necessary to be careful with the small eigenvalues and in particular distinguish between the residual and cuspidal spectrum. This distinction, which was not necessary in [29], does not show up in the statement of the theorem but is necessary for the proof to be rigorous. Given that most of the details appear in [29] and [21] we will only give an outline of the proof and highlight certain points which are of particular importance.

It is well-known that the Selberg zeta function, $Z(s)$, for the group $G$ can be extended to a meromorphic function which satisfies a functional equation of the form

$$
Z(s)=\eta(s) \varphi(s)^{-1} Z(1-s)
$$

where $\varphi(s)$ is the scattering determinant (see Section 3) and $\eta(s)$ is an explicit function, given by its logarithmic derivative $\mathcal{C}(s)=\left(\eta^{\prime} / \eta\right)(s)$ (cf. e.g. [21, p. 464]). Since discrete eigenvalues of the Laplacian correspond to zeros of $Z(s)$ it is possible to prove the theorem by comparing the zeros and poles of $Z(s)$ and of $Z(1-s)$ within a certain rectangle. More precisely, let $3 / 2>K>1, T \geq 1$ and let $R(K, T)$ be the rectangle with corners $K-i T, K+i T, 1-K-i T, 1-K+i T$. Assume that $K$ and $T$ are chosen so that $Z$ has no zeros or poles on the boundary $\partial R(K, T)$. Recall that the relationship between the spectral parameters and Laplace eigenvalues is given by $s \mapsto \lambda=s(1-s)$. It follows that, except for the residual eigenvalues, the count of spectral parameters inside the rectangle is double of the eigenvalue count. The reason for excluding residual eigenvalues is of course that if $s$ is a pole of $\varphi(s)$ then $1-s$ is a zero, due to the fact that $\varphi(s) \varphi(1-s)=1$.

If $\mathcal{D}(s)=\left(Z^{\prime} / Z\right)(s)$, it follows immediately from [21, p. 498, Thm. 5.3] that

$$
\frac{1}{2 \pi i} \int_{\partial R(K, T)} \mathcal{D}(s) d s=N_{\mathrm{res}}+2 N_{\mathrm{exc}}+2 N(T)+2 Q(T)+2 g-2+e_{\infty}-C,
$$

where $Q(T)$ is the number of zeros of $\varphi(s)$ inside $R(K, T)$ with $0<\Im(s) \leq T$ and $C=\operatorname{Tr}(I-\Phi(1 / 2)) / 2$. Let $P:=P(K, T)$ be the polygonal path through the vertices $1 / 2-i T, K-i T, K+i T$ and $1 / 2+i T$. From the functional equation we see that

$$
\frac{1}{2 \pi i} \int_{\partial R(K, T)} \mathcal{D}(s) d s=2 S(T)-\frac{1}{2 \pi i} \int_{P} \mathcal{C}(s) d s+\frac{1}{2 \pi i} \int_{P} \frac{\varphi^{\prime}}{\varphi}(s) d s,
$$

where

$$
S(T)=\frac{1}{2 \pi i} \int_{P} \mathcal{D}(s) d s=-\frac{1}{\pi} \Im \int_{1 / 2}^{\infty} \mathcal{D}(\sigma+i T) d \sigma .
$$

Since $\varphi$ has $N_{\text {res }}$ poles (cf. e.g. [21, pp. 76-77]) and $2 Q(T)$ zeros inside the region enclosed by $P$ and the line $\sigma=1 / 2$ it follows from the residue theorem that

$$
\frac{1}{2 \pi i} \int_{\partial R(K, T)} \mathcal{D}(s) d s=2 S(T)-\frac{1}{2 \pi i} \int_{P} \mathcal{C}(s) d s+2 Q(T)-N_{\text {res }}-2 M(T)
$$


and we are left to work out the integral involving $\mathcal{C}(s)$. By using elementary trigonometric relations it follows from [21, pp. 499-500] that

$$
\mathcal{C}(s)=\frac{h \pi}{3}(s-1 / 2) \tan (\pi(s-1 / 2))+E(s)+e_{\infty}(2 \ln 2+\psi(s+1 / 2)+\psi(3 / 2-s)),
$$

where

$$
E(s)=-e_{2} \frac{\pi}{2 \cos (\pi(s-1 / 2))}-e_{3} \frac{4 \pi}{3 \sqrt{3}}\left\{\frac{\cos (\pi / 3(s-1 / 2))}{\cos (\pi(s-1 / 2))}\right\} .
$$

Observe that each elliptic fixed point of order 3 correspond to two elliptic conjugacy classes with rotations of angles $\pi / 3$ and $2 \pi / 3$. It is now easy to see that $\mathcal{C}(s)$, inside the region bounded by $P$ and the line $\Re s=1 / 2 \mathcal{C}(s)$, has a single simple pole at $s=1$ and

$$
\operatorname{Res}_{s=1} \mathcal{C}(s)=-\frac{h}{6}+\frac{1}{2} e_{2}+\frac{2}{3} e_{3}=2-2 g-e_{\infty} .
$$

For the last equality we use the Gauss-Bonnet theorem (see (12) for the formulation in our setting). Using the residue theorem again and combining (10) and (11) we find that

$$
2 N_{\mathrm{res}}+2 N_{\mathrm{exc}}+2 N(T)+2 M(T)=C+2 S(T)-\frac{1}{\pi} \int_{0}^{T} \mathcal{C}(1 / 2+i t) d t .
$$

Finally, using the expression for $\mathcal{C}(s)$ above, we write

$$
\frac{1}{2 \pi} \int_{0}^{T} \mathcal{C}(1 / 2+i t) d t=I_{I d}+I_{E}+I_{P}
$$

The three integrals are evaluated exactly as in [29], except that we will refine the bound for the elliptic terms and also replace certain bounds by rational numbers (using their continued fraction expansions). More precisely we obtain

$$
I_{I d}=-\frac{h}{12} T^{2}+\frac{h}{144}+h g_{I d}(T)
$$

where

$$
\left|g_{I d}(T)\right| \leq \frac{1}{3} \frac{2 \pi+1}{4 \pi^{2} e^{2 \pi}} \cdot \frac{1}{T}<\frac{1}{8707} \cdot \frac{1}{T} .
$$

For the elliptic integrals, if we write $I_{E}=e_{2} I_{E, 2}+2 e_{3} I_{E, 3}$ then $I_{E, 2}=-\frac{1}{8}+g_{E, 2}(T)$ and $I_{E, 3}=-\frac{1}{9}+g_{E, 3}(T)$. Since the only orders of elliptic elements here are 2 and 3 we use a simple change of variables to evaluate the remainder integrals explicitly:

$$
\begin{aligned}
g_{E, 2}(T)= & \frac{1}{4} \int_{T}^{\infty} \frac{1}{\cosh (\pi t)} d t=\frac{1}{4}\left[\frac{2 \arctan (\tanh (\pi t / 2))}{\pi}\right]_{T}^{\infty}= \\
& =\frac{1}{2 \pi}\left(\frac{\pi}{4}-\arctan (\tanh (\pi T / 2))\right)
\end{aligned}
$$

and similarly

$$
g_{E, 3}(T)=\frac{1}{3 \sqrt{3}} \int_{T}^{\infty} \frac{\cosh (\pi t / 3)}{\cosh (\pi t)} d t=\frac{1}{3 \pi}\left(\frac{\pi}{3}-\arctan (\sqrt{3} \tanh (\pi T / 3))\right) .
$$


A straight-forward application of Taylor's theorem with reminder shows that

$$
\begin{gathered}
|\arctan (\tanh (\pi T / 2))-\pi / 4| \leq|\tanh (\pi T / 2)-1| /\left(\tanh (\pi T / 2)^{2}+1\right) \text { and } \\
|\arctan (\sqrt{3} \tanh (\pi T / 3))-\pi / 3| \leq|\sqrt{3} \tanh (\pi T / 3)-\sqrt{3}| /\left(3 \tanh (\pi T / 3)^{2}+1\right) .
\end{gathered}
$$

Since we assume that $T \geq 1$ we can bound $\tanh (\pi T / 2)^{2}+1$ and $3 \tanh (\pi T / 3)^{2}+1$ from below by $\tanh (\pi / 2)^{2}+1>11 / 6$ and $\tanh (\pi / 3)^{2}+1>14 / 5$. The trivial inequality $e^{a T}+1>T\left(e^{a}+1\right)$ (for any $a>2$ and $T \geq 1$ ) then gives the following bounds

$$
\left|g_{E, 2}(T)\right| \leq \frac{1}{2 \pi} \frac{6}{11}|\tanh (\pi T / 2)-1|=\frac{6}{11 \pi} \frac{1}{e^{\pi T}+1}<\frac{6}{11 \pi} \frac{1}{e^{\pi}+1} \cdot \frac{1}{T}<\frac{1}{139} \cdot \frac{1}{T}
$$

and

$$
\left|g_{E, 3}(T)\right| \leq \frac{5}{14 \sqrt{3} \pi}|\tanh (\pi T / 3)-1|=\frac{5}{7 \sqrt{3} \pi} \frac{1}{e^{\frac{2 \pi T}{3}}+1} \leq \frac{5}{7 \sqrt{3} \pi} \frac{1}{e^{\frac{2 \pi}{3}}+1} \cdot \frac{1}{T}<\frac{1}{67} \cdot \frac{1}{T} .
$$

Finally, the parabolic term is $I_{P}=\frac{1}{\pi} \Im \log \Gamma(1+i T)+\frac{1}{\pi} T \log 2$ and using Stirling's formula as given by e.g. Stieltjes [61] and using estimates as in [29] it follows that

$$
I_{P}=\frac{1}{\pi} T \ln (2 T / e)+\frac{1}{4}+g_{P}(T)
$$

where $\left|g_{P}(T)\right| \leq \frac{49}{\pi 45} \cdot \frac{1}{T}<\frac{26}{75} \cdot \frac{1}{T}$. Taking all terms together we now find that

$$
\begin{aligned}
N_{\text {res }}+N_{\text {exc }}+N(T)+M(T) & =S(T)-\frac{1}{2 \pi} \int_{0}^{T} \mathcal{C}(1 / 2+i t) d t \\
& =\frac{h}{12} T^{2}-\frac{e_{\infty}}{\pi} T \ln (2 T / e)+c_{G}+g(T)+S(T),
\end{aligned}
$$

where

$$
c_{G}=-\frac{h}{144}+\frac{e_{2}}{8}+\frac{2 e_{3}}{9}-\frac{e_{\infty}}{4}+C / 2
$$

and

$$
|g(T)| \leq\left(h \frac{1}{8707}+e_{2} \frac{1}{139}+2 e_{3} \frac{1}{67}+e_{\infty} \frac{26}{75}\right) \cdot \frac{1}{T}
$$

Example 5. For the modular group we have $h=e_{2}=e_{3}=e_{\infty}=1$, there is precisely one residual eigenvalue $(\lambda=0)$ and $\varphi(1 / 2)=-1$ so $C=1$. It follows that

$$
N(T)+M(T)+1=\frac{1}{12} T^{2}-\frac{1}{\pi} T \ln (2 T / e)+\frac{85}{144}+g(T)+S(T)
$$

with $|g(T)|<0.384 \cdot \frac{1}{T}$. By considering the individual terms in [29] we see that

$$
M(T)=\frac{T}{\pi} \log (T /(e \pi))+1 / 2+g_{M}(T)+S_{M}(T)
$$

where $\left|g_{M}(T)\right|<3091 /(1800 \pi)$ and $\int_{0}^{T} S_{M}(t) d t=O\left(T / \log ^{2} T\right)$. We therefore recover, for instance [29, Cor. 14] with a slight improvement in the bound $\left|G_{1}(T)\right|<c / T$ with $c=0.931$ instead of 0.977 . 


\section{Subgroups of the Modular Group}

There are at least two different efficient approaches to computing with finite index subgroups of the modular group. One approach is based on Farey symbols and was introduced by Kulkarni [32] and later on used for computations by Kurth and Long [36]. We chose a second approach, based on representing subgroups of the modular group by pairs of permutations. This method was first used by Millington to study subgroups of the modular group [43] in general and cycloidal groups, that is, groups with one cusp, in more detail [42]. The same approach was also used by Atkin and Swinnerton-Dyer [3], who in fact implemented an algorithm based on this representation and used it to obtain information about genus zero noncongruence subgroups up to index 18. Additionally they also introduced another method, based on the modular function $j(z)=q^{-1}+744+196884 q+\cdots$, where $q=e^{2 \pi i z}$ and the fact that there exists a necessary and sufficient condition for an algebraic function of $j$ to be a modular function on a subgroup [3, Thm. 1].

\subsection{Modular forms on noncongruence subgroups}

The main purpose of Atkin and Swinnerton-Dyer [3] was not only to study noncongruence subgroups, but to compute modular forms. The most important result of [3] was undoubtedly the observation of certain congruences relations between Fourier coefficients of modular forms on noncongruence groups and modular forms on congruence groups. Nowadays these congruences are usually called "Atkin and Swinnerton-Dyer (ASD) congruences" and has been the subject of a large number of papers where partial results have been proven $[2,38,39]$. Another interesting observation was that in all the cases they considered the modular functions for noncongruence subgroups could all be seen to have rational Fourier coefficients with unbounded denominators, whereas on congruence groups it is always possible to choose a basis of modular forms or functions with rational integral Fourier coefficients. This observation inspired the folklore "Unbounded denominators conjecture" saying that having Fourier coefficients with unbounded denominators precisely characterizes modular functions and forms for noncongruence subgroups. This conjecture has been proven in special cases, see, for example [15, 34, 35, 37].

From a computational point of view Maass forms (on any subgroup) and modular forms on noncongruence subgroups share a major disadvantage compared to modular forms on congruence groups. Namely that their Fourier coefficients can in general not be interpreted as eigenvalues of Hecke operators acting on computationally tractable spaces. For modular forms on congruence groups the most efficient (general) algorithms today uses the Hecke algebra, and in particular the realization of this algebra in terms of a family of commuting and normal linear operators acting on a space of modular symbols. A good introduction to computing congruence modular forms is given by Stein [58].

There is still an associated space of modular symbols for noncongruence groups [33] but there are not sufficiently many (non-trivial) Hecke operators available [7, 55]. Alternative methods for computing congruence modular forms, for example using explicit generators for the ring of modular forms (e.g. Eisenstein series, eta or theta functions) or Eichler-Selberg type trace formulas are also not in general immediately applicable to noncongruence subgroups. One important exception is when the space of modular forms of weight $k$ is one-dimensional, then it is sometimes possible to give an explicit basis (consisting of one function) in terms of a fractional power of a rational function of the 
Dedekind eta function, $\eta(z)$. This is in particular true for so-called character groups, who are defined precisely by such functions. The case of modular functions, however, seems to be slightly easier by using either the methods of Atkin and Swinnerton-Dyer [3] as well as new algorithms by Monien [45] based on Farey fractions and ideas by Rademacher and Zuckerman [49]. In fact, Monien also adapted these algorithms to Maass cusp forms by using standard numerical methods for partial differential equations.

It is actually also possible to use techniques from differential equations to study holomorphic modular forms. This has been successfully exploited in the setting of vectorvalued modular forms by, for instance, Cameron and Mason [17], and Marks [41]. Using these techniques and the identification of modular forms on subgroups with vector-valued modular forms they obtained interesting structural results as well as further proofs of the unbounded denominator conjecture in specific cases.

For Maass forms the situation is that, even when there exists an infinite family of Hecke operators these can in general not be interpreted as operators acting on a finite dimensional space. Except for certain spaces of Maass forms corresponding to lifts of Hecke Grössencharakters the only viable approach to Maass forms on any kind of subgroups of the modular group has been to use computational methods. The method we are using to compute Maass cusp forms is very easy to adapt to also compute holomorphic modular forms. In fact, the methods used by Atkin and Swinnerton-Dyer [3] as well as Richards [50] are similar to the first version of the automorphy method used by Hejhal [22] to compute Maass cusp forms for the modular group and other Hecke triangle groups.

\subsection{Subgroups}

It is well-known that $\mathrm{PSL}_{2}(\mathbb{Z})$ is generated by the maps $S: z \mapsto-z^{-1}$ and $T: z \mapsto$ $z+1$, and the only relations are $S^{2}=R^{3}=\mathbb{1}_{2}$, where $R=S T$ and $\mathbb{1}_{2}$ is the identity:

$$
S=\left(\begin{array}{cc}
0 & -1 \\
1 & 0
\end{array}\right), \quad T=\left(\begin{array}{cc}
1 & 1 \\
0 & 1
\end{array}\right) \quad \text { and } \quad R=\left(\begin{array}{cc}
0 & -1 \\
1 & 1
\end{array}\right) .
$$

Let $G \leqslant \mathrm{PSL}_{2}(\mathbb{Z})$ be a subgroup of finite index $h$ and $e_{2}$ and $e_{3}$ inequivalent elliptic vertices of order 2 and 3 respectively and $e_{\infty}$ inequivalent cusps. The genus $g$ of the quotient orbifold, $\Gamma \backslash \mathcal{H}$, is then given by

$$
g=1+\frac{1}{2}\left(\frac{h}{6}-e_{\infty}-\frac{1}{2} e_{2}-\frac{2}{3} e_{3}\right),
$$

and we say that the signature of $\Gamma$ is $\left(h ; g, e_{\infty}, e_{2}, e_{3}\right)$ (in [43] this is called the type of $\Gamma$ ) and it is known that signatures consistent with (12) correspond to subgroups of $\mathrm{PSL}_{2}(\mathbb{Z})$. More precisely, we have

Lemma 6 ([43, Thm. 2]). For every set of integers $h>0, e_{\infty}>0, e_{2} \geq 0, e_{3} \geq 0, g \geq 0$ consistent with (12) there exists a subgroup of the modular group with signature

$$
\left(h ; g, e_{\infty}, e_{2}, e_{3}\right) .
$$

Let $p_{j} \in \mathbb{Q} \cup\{\infty\}$ be a cusp of $G$ with stabilizer $\Gamma_{p_{j}}$ generated by the parabolic element $T_{p_{j}}$. The cusp width of $p_{j}$ is defined as the positive integer $h_{j}$ such that $T_{p_{j}}$ is conjugate in $\mathrm{PSL}_{2}(\mathbb{Z})$ to $T^{ \pm h_{j}}: z \mapsto z \pm h_{j}$. If the fundamental domain $\mathcal{F}_{G}$ is given by $h$ 
copies of $\mathcal{F}_{1}$ then $h_{j}$ is the number of those copies which meet at $p_{j}$ and it is clear that $h=h_{1}+\cdots+h_{e_{\infty}}$. The generalized level of $\Gamma$ is defined in the spirit of Wohlfahrt [72] as the least common multiple of the numbers $h_{1}, \ldots, h_{e_{\infty}}$ and if $\Gamma$ is a congruence subgroup then the generalized level is the same as the usual level.

\subsection{Permutations and subgroups}

If $G$ is any group and $H \leqslant G$ is a finite index subgroup then there is a natural representation of $H$ as a permutation group acting on the (left or right) cosets of $H$ in $G$. This is the foundation for Millington's method to work with subgroups of the modular group [43], which we will now describe in more detail.

An intuitive geometric way to view the permutation representation of a subgroup $G \leqslant \mathrm{PSL}_{2}(\mathbb{Z})$ of index $h$ is to consider a fixed fundamental domain $\mathcal{F}_{\Gamma}=\cup_{h} V_{i} \mathcal{F}_{1}$ for $\Gamma$ and study the action of the generators $S$ and $R$ on the $h$ copies $V_{1} \mathcal{F}_{1}, \ldots, V_{h} \mathcal{F}_{1}$. In this way we obtain an associated pair of permutations $\sigma_{S}, \sigma_{R} \in \mathrm{S}_{h}$ of order 2 and 3 , respectively. These permutations now contain all information about the side-pairing transformations of $\mathcal{F}_{\Gamma}$ and by Poincaré's theorem, all information necessary to recover the group $\Gamma$. We will now see which such pairs, conversely, correspond to subgroups of the modular group.

Definition 7. If $\sigma_{S}, \sigma_{R} \in \mathrm{S}_{h}$ we say that the pair $\left(\sigma_{S}, \sigma_{R}\right)$ is legitimate if

- $\sigma_{S}^{2}=\sigma_{R}^{3}=i d$, and

- the subgroup, $\left\langle\sigma_{S}, \sigma_{R}\right\rangle \leqslant \mathrm{S}_{h}$ generated by $\sigma_{S}$ and $\sigma_{R}$ is transitive on $\{1,2, \ldots, h\}$.

Two legitimate pairs $\Sigma=\left(\sigma_{S}, \sigma_{R}\right)$ and $\Sigma^{\prime}=\left(\sigma_{S}^{\prime}, \sigma_{R}^{\prime}\right)$ are said to be equivalent, denoted by $\Sigma \sim \Sigma^{\prime}$, if there exists a permutation $\pi \in \mathrm{S}_{h}$ such that $\Sigma^{\pi}=\Sigma^{\prime}$ where $\Sigma^{\pi}=$ $\left(\pi^{-1} \sigma_{S} \pi, \pi^{-1} \sigma_{R} \pi\right)$. If, in addition, $\pi(1)=i d$, then we say that the pairs are equivalent (mod 1), which we denote by $\Sigma \sim_{1} \Sigma^{\prime}$. By abuse of notation we will sometimes let $\Sigma$ also denote the subgroup $\left\langle\sigma_{S}, \sigma_{R}\right\rangle$.

The fundamental theorem by Millington is the following.

Theorem 8. (Millington [43, Thm. 1]) There is a one-to-one correspondence between equivalence classes (mod 1) of legitimate pairs $\left(\sigma_{S}, \sigma_{R}\right)$ in $S_{h}$ and subgroups $G$ of index $h$ in $\mathrm{PSL}_{2}(\mathbb{Z})$.

Furthermore, $G$ has signature $\left(h ; g, e_{\infty}, e_{2}, e_{3}\right)$ and cusp widths $h_{1}, \ldots, h_{e_{\infty}}$ if and only if $\sigma_{S}$ and $\sigma_{R}$ fixes precisely $e_{2}$ and $e_{3}$ elements, $\sigma_{T}=\sigma_{S} \sigma_{R}$ has $e_{\infty}$ disjoint cycles of lengths $h_{1}, h_{2}, \ldots, h_{e_{\infty}}$ and $g$ satisfies (12).

The subgroup, $G$, of $\mathrm{PSL}_{2}(\mathbb{Z})$ corresponding to the legitimate pair $\left(\sigma_{S}, \sigma_{R}\right)$ in the theorem is defined by

$$
G=\left\{\gamma \in \mathrm{PSL}_{2}(\mathbb{Z}) \mid \phi(\gamma)(1)=1\right\},
$$

where $\phi: \mathrm{PSL}_{2}(\mathbb{Z}) \rightarrow \mathrm{S}_{h}$ is the homomorphism defined by $\phi(S)=\sigma_{S}$ and $\phi(R)=\sigma_{R}$ and extended by $\phi(A B)=\phi(A) \phi(B)$. In particular $\phi(T)=\phi(S R)$. Observe that any set $\left\{V_{1}, \ldots, V_{h}\right\} \subset \mathrm{PSL}_{2}(\mathbb{Z})$ satisfying $\phi\left(V_{j}\right)(1)=j$ is a set of right coset representatives for $G \backslash \mathrm{PSL}_{2}(\mathbb{Z})$. 
Theorem 9. Let $G$ and $G^{\prime}$ correspond to the legitimate pairs $\Sigma$ and $\Sigma^{\prime}$. Then $G$ and $G^{\prime}$ are conjugate in $\mathrm{PSL}_{2}(\mathbb{Z})$ if and only if $\Sigma \sim \Sigma^{\prime}$.

Proof. Let $\phi$ denote the homomorphism defined by $\Sigma$. If $A \in \mathrm{PSL}_{2}(\mathbb{Z})$ then

$$
A G A^{-1}=\left\{\gamma \in \mathrm{PSL}_{2}(\mathbb{Z}) \mid \phi(A)^{-1} \phi(\gamma) \phi(A)(1)=1\right\}
$$

in other words, the group $A G A^{-1}$ corresponds to the legitimate pair $\phi(A)^{-1} \Sigma \phi(A)=$ $\Sigma^{\phi(A)}$. Conversely, if $\Sigma^{\prime}=\Sigma^{\tau}$ for some $\tau \in \mathrm{S}_{h}$ then it is easy to verify that $G^{\prime}=A G A^{-1}$ for any $A$ with $\phi(A)(1)=\tau(1)$.

Definition 10. If $G$ is a subgroup of $\operatorname{PSL}_{2}(\mathbb{Z})$ then $(G)$ denotes the set of $\mathrm{PSL}_{2}(\mathbb{Z})$ conjugates of $G$,

$$
(G)=\left\{A G A^{-1} \mid A \in \mathrm{PSL}_{2}(\mathbb{Z})\right\} .
$$

If $G \leqslant \mathrm{PSL}_{2}(\mathbb{Z})$ corresponds to the pair $\Sigma$ and $A \in \mathrm{PSL}_{2}(\mathbb{Z})$ then $A G A^{-1}$ corresponds to the pair $\Sigma^{\sigma_{j}}$ where $j=\phi(A)^{-1}(1)$ and $\sigma_{j}=(1 j)$. It is easily seen that all conjugates of $G$ can be obtained by conjugating $\Sigma$ with $\sigma_{j}$ for $j=2, \ldots, h$. It follows that the number of elements in $(G)$ divides $h$.

\subsection{Block systems and supergroups}

To prove that we actually have newforms we have to find a way to check for supergroups in $\mathrm{PSL}_{2}(\mathbb{Z})$; fortunately there is a well-known combinatorial method to find all of these. This algorithm is essentially what is also implemented in Sage.

Definition 11. A block system for $H \leqslant \mathcal{S}_{h}$ is an equivalence relation, $\equiv$, on $\{1,2, \ldots, h\}$ defined by the condition

$$
x \equiv y \Leftrightarrow \gamma(x) \equiv \gamma(y) \quad \forall \gamma \in H
$$

An equivalence class, $[x]=\{y \mid y \equiv x\}$, is called a block. For any $H \leqslant \mathcal{S}_{h}$ there are two trivial block systems: The block system with $h$ blocks and the block system with 1 block. It is easy to see that if the group $H$ is transitive, then all blocks of $H$ must have the same number of elements. The following proposition can be used to determine whether non-trivial supergroups exist or not.

Proposition 12. Let the legitimate pair $\left(\sigma_{S}, \sigma_{R}\right)$ correspond to the group $G<\mathrm{PSL}_{2}(\mathbb{Z})$. Then there exists an supergroup $G^{\prime}$ with $G<G^{\prime}<\mathrm{PSL}_{2}(\mathbb{Z})$ if and only if the subgroup $\left\langle\sigma_{S}, \sigma_{R}\right\rangle \leqslant \mathcal{S}_{h}$ supports a non-trivial block system.

Proof. Suppose that $G^{\prime}$ is an supergroup of $G$ with $G<G^{\prime}<\operatorname{PSL}_{2}(\mathbb{Z})$, let $h$ be the homomorphism defined by $\left(\sigma_{S}, \sigma_{R}\right)$ and set $\mathcal{B}=\left\{\phi(\gamma)(1) \mid \gamma \in G^{\prime}\right\} \subseteq\{1,2, \ldots, h\}$. Since $G^{\prime} \neq G$ and $G^{\prime} \neq \mathrm{PSL}_{2}(\mathbb{Z})$ it follows that $\mathcal{B}$ at least two and at most $h-1$ elements. It is easy to verify that the equivalence relation defined by

$$
x \equiv y \quad \Longleftrightarrow \quad \exists \gamma \in \mathrm{PSL}_{2}(\mathbb{Z}):[\phi(\gamma)(x) \in \mathcal{B} \text { and } \phi(\gamma)(y) \in \mathcal{B}]
$$

gives a block system with $\mathcal{B}=[1]$. Conversely, if $\equiv$ is a non-trivial block system for the group $\left\langle\sigma_{S}, \sigma_{R}\right\rangle$, then

$$
G^{\prime}=\left\{\gamma \in \mathrm{PSL}_{2}(\mathbb{Z}) \mid \phi(\gamma)(1) \equiv 1\right\}
$$

is a non-trivial supergroup of $G$. 
Remark 13. It is easy to see that if $\left\langle\sigma_{E}, \sigma_{R}\right\rangle$ supports a non-trivial block system with $d$ blocks $\mathcal{B}_{1}, \mathcal{B}_{2}, \ldots, \mathcal{B}_{d}$ then corresponding supergroup $G^{\prime}$ has index $d$ in $\operatorname{PSL}_{2}(\mathbb{Z})$. Furthermore, $G^{\prime}$ is given by legitimate pair $\left(\sigma_{E}^{\prime}, \sigma_{R}^{\prime}\right)$ in $\mathcal{S}_{d}$ given by the action that $\sigma_{S}$ and $\sigma_{R}$ induce on the block structure.

To verify whether a given equivalence relation $\equiv$ is a block system for the group $\left\langle\sigma_{E}, \sigma_{R}\right\rangle$ or not we only need to check if the following condition holds:

$$
\forall x, y \in\{1, \ldots, h\}: \quad x \equiv y \quad \Longleftrightarrow \sigma_{S}(x) \equiv \sigma_{S}(y) \quad \Longleftrightarrow \quad \sigma_{R}(x) \equiv \sigma_{R}(y) .
$$

Since the permutation $\sigma_{R}$ has order 3 we only need to consider 1 and 3-cycles and the latter are easy to deal with by using the following remark.

Remark 14. Suppose that $(a b c)$ is a 3-cycle of $\sigma_{R}$. Then, if $a \equiv b$, we must have $\sigma_{R}(a)=b \equiv \sigma_{R}(b)=c$, and similarly if $a \equiv c$ or $b \equiv c$. Hence either $a, b, c$ are in the same block or they are all in different blocks.

We will now give an explicit example of how to checks for block systems.

Example 15. Consider the group $G=\Gamma(10 ; 0,3,0,1 ; 20,1)$ given by

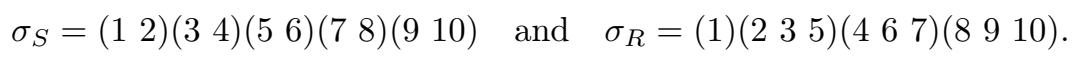

Since $h=10$ there are two possibilities for non-trivial block systems. There are either two blocks of length 5 or 5 blocks of length 2. It follows immediately from Remark 14 that there can not be 2 blocks of length 5 . Suppose now that we have 5 blocks of length 2 and let $x \in[1]$ with $x \neq 1$. Then $\sigma_{R}(x) \equiv \sigma_{R}(1)=1$, hence $\sigma_{R}(x) \in$ [1] so that $\sigma_{R}(x)=x$ or $\sigma_{R}(x)=1$. Since the only fixed element of $\sigma_{R}$ is 1 it follows that $x=1$, which is a contradiction. Hence there can not exist any non-trivial block system and we conclude that there is no non-trivial supergroup on $G$. In view of Theorem 26 we have shown that there exist an infinite number of linearly independent newforms for $G$.

The groups we are primarily interested in are those which are not congruence subgroups and that possess a symmetry of the form $\alpha: z \mapsto k-\bar{z}$ which preserves cusp classes. In the range covered by Table 2 this includes all groups $G$ with $h_{G}=1$, i.e. $\left(G^{*}\right)=(G)$, except for $\Gamma(9 ; 0,3,1,0 ; 7,1)$ and $\Gamma(10 ; 0,3,0,1 ; 8,1)$, where the map which conjugates $G$ to $G^{*}$ permute the cusp classes. Out of the remaining subgroups only $\Gamma(9 ; 0,2,3,0 ; 6,1)$, $\Gamma(9 ; 0,3,1,0 ; 6,1)$ and $\Gamma(9 ; 0,3,1,0 ; 12,1)$ have non-trivial supergroups. In Table 2 we indicate the number of supergroups in the column $n_{\text {sup }}$.

\subsection{Congruence subgroups}

Let $G \leqslant \mathrm{PSL}_{2}(\mathbb{Z})$ be a subgroup of index $h$ and generalized level $N$. If $G$ is a congruence group then it has level $N$ and all $\mathrm{PSL}_{2}(\mathbb{Z})$ conjugates of $G$ are also congruence subgroups of level $N$. The index of $\Gamma(N)$ in $\mathrm{PSL}_{2}(\mathbb{Z})=\Gamma(1)$ is equal to 6 if $N=2$ and

$$
[\Gamma(N): \Gamma(1)]=\frac{1}{2} N^{3} \prod_{p \mid N}\left(1-p^{-2}\right) \quad \text { if } N \geq 3,
$$

where the product is taken over all prime divisors of $N$. It is clear that if $h$ does not divide $[\Gamma(N): \Gamma(1)]$ then $G$ is not a congruence group. 
Example 16. Consider the groups of signature $(7 ; 0,2,1,1)$ and generalized levels 6,10 and 12 in Table 2. From (13) we see that the index of $\Gamma(6), \Gamma(10)$ and $\Gamma(12)$ are 72 , 360 and 1152. Since neither of these numbers are divisible by 7 it follows that all three groups are noncongruence subgroups.

Example 17. Consider the group $G$ of signature $(9 ; 0,2,3,0)$ and generalized level 8 in Table 2. The index of $\Gamma(8)$ is 192 , and since 9 does not divide 192 the group $G$ must be a noncongruence subgroup.

Clearly this fact can not tell us that a given group is a congruence subgroup, and in general it can not even help us to determine when a given group is not a congruence subgroup. For instance, out of the three groups of signature $(8 ; 0,1,2,2)$ and generalized level 8 in Table 2, only one is a congruence group. Thus, not even the signature together with the generalized level are not sufficient to decide if a group is a congruence group or not.

Fortunately there is a theorem by Hsu [24, Thm. 3.1] that give us necessary and sufficient conditions on the legitimate pair $\left(\sigma_{E}, \sigma_{R}\right)$ in order for the corresponding subgroup to be a congruence subgroup (see also [31] for the case of odd subgroups of $\mathrm{SL}_{2}(\mathbb{Z})$ ). The theorem of $\mathrm{Hsu}$ is implemented in Sage and it can easily be translated to our setting.

\section{Old- and Newform Theory for Noncongruence Subgroups}

The main goal with this section is to give an explicit testable condition for the existence of an infinite number of genuinely non-congruence Maass cusp forms, meaning that they are not coming from a congruence supergroup. We will in fact prove the stronger statement that there are an infinite number of "new" Maass cusp forms which are not associated with any supergroup. For this purpose we will first give a proper explanation of what "new" means in this context.

\subsection{Old and newforms}

The theory of oldforms and newforms, as introduced by Atkin and Lehner [1] plays a crucial role in the structural theory of modular forms on congruence subgroups. The generalization to Maass forms on congruence subgroups is immediate (see e.g. [65, 68]). A key result is the orthogonal decomposition, with respect to the Petersson inner product, of the space of Maass cusp forms for the group $\Gamma_{0}(N)$ :

$$
\mathcal{M}^{0}(N)=\mathcal{M}^{\text {new }}(N) \oplus \mathcal{M}^{\text {old }}(N)
$$

where the oldform space, $\mathcal{M}^{\text {old }}(N)$, can be written in terms of lower level spaces as

$$
\mathcal{M}^{\text {old }}(N)=\bigoplus_{M<N, d \mid N / M} B(d) \mathcal{M}^{\text {new }}(M)
$$

and $B(d): \tau \mapsto d \tau$ is the map given by $\left(\begin{array}{ll}d & 0 \\ 0 & 1\end{array}\right) \in \mathrm{PGL}_{2}(\mathbb{Q})$. This theory and its applications is well understood for congruence subgroups but the analogue for noncongruence subgroups has not been studied before and we will briefly outline the relevant aspects.

Our main motivation for studying old and new Maass cusp forms is that we would like to understand the multiplicities with which the spectrum of supergroups appear within 
their subgroups. In other words, we want to understand how Maass cusp forms on $G$ can be defined using ones on $G^{\prime}$ in a natural way. If $G<G^{\prime} \leqslant \mathrm{PSL}_{2}(\mathbb{Z})$ then $\mathcal{M}\left(G^{\prime}\right) \subseteq \mathcal{M}(G)$ and the inclusion map gives one possibility but it is not immediately clear whether there are other such maps and to investigate this we first need a few definitions and results.

The group of complex (holomorphic and anti-holomorphic) automorphisms of the upper half-plane is $\mathrm{PGL}_{2}(\mathbb{R})$ and a reasonable choice of "natural maps" in this setting is therefore given by maps of the form $f \mapsto f \mid \alpha$ where $\alpha \in \mathrm{PGL}_{2}(\mathbb{R})$. For this to map $\mathcal{M}\left(G^{\prime}\right)$ into $\mathcal{M}(G)$ we need $\alpha$ in the so-called conjugator of $G$ in $G^{\prime}$ :

$$
C\left(G^{\prime}, G\right)=\left\{\alpha \in \mathrm{PGL}_{2}(\mathbb{R}) \mid \alpha G \alpha^{-1} \leqslant G^{\prime}\right\},
$$

which we use to define old and new cusp forms for noncongruence groups. The conjugator of the group $G^{\prime}$ in itself, $C\left(G^{\prime}\right)=C\left(G^{\prime}, G^{\prime}\right)$ consists of all symmetries of $G^{\prime}$, or in other words all $\beta \in \mathrm{PGL}_{2}(\mathbb{R})$ such that $\beta G^{\prime} \beta^{-1}=G^{\prime}$. Since we are interested in different maps $\alpha: f \mapsto f \mid \alpha$ we are not interested in the full conjugator, but rather the double quotient

$$
\tilde{C}\left(G^{\prime}, G\right)=C\left(G^{\prime}\right) \backslash C\left(G, G^{\prime}\right) / C(G) .
$$

Definition 18. If $G$ is a noncongruence subgroup then the old space of Maass cusp forms on $G$ is defined as

$$
\mathcal{M}^{\text {old }}(G)=\sum_{G<G^{\prime}} \sum_{\alpha \in \tilde{C}\left(G^{\prime}, G\right)} \alpha \mathcal{M}\left(G^{\prime}\right),
$$

and it elements are called oldforms. We remark that the sum is in general not direct. The new space, $\mathcal{M}^{\text {new }}(G)$, is defined as the orthogonal complement of the old space with respect to the Petersson inner product. For noncongruence groups we also say that elements of the new space are newforms if they are normalized so that the first non-vanishing Fourier coefficient at the cusp $\infty$ is equal to 1 .

It is known that a noncongruence subgroup has only at most a finite number of nontrivial Hecke operators and in particular all Hecke operators of index not dividing the generalized level are trivial. Cf. e.g. Scholl [55] and Berger [7]. Hence this definition of newform is consistent with that for congruence groups, see e.g. [1]. It is also consistent with the notion of genuinely new Maass waveforms introduced in [66].

By using the description of the set $\tilde{C}\left(G^{\prime}, G\right)$ below it is also easy to verify that the definition of old space agrees with the definition above for congruence subgroups. For instance if $G=\Gamma_{0}(N)$ and $G^{\prime}=\mathrm{SL}_{2}(\mathbb{Z})$ then $\tilde{C}\left(G^{\prime}, G\right)=\{B(d)|d| N\}$.

Let $G<G^{\prime} \leqslant \mathrm{PSL}_{2}(\mathbb{Z})$ be fixed for the remainder of this section. In general $C\left(G^{\prime}, G\right)$ is not a group but it is contained (as a set) within the commensurator:

$$
\operatorname{Comm}(G)=\left\{\alpha \in \mathrm{PGL}_{2}(\mathbb{R}) \mid G \cap \alpha G \alpha^{-1} \leqslant G, G \cap \alpha G \alpha^{-1} \leqslant \alpha G \alpha^{-1} \text { (finite index) }\right\}
$$

and the group generated by all the elements of $C\left(G^{\prime}, G\right)$ is a subgroup of $\operatorname{Comm}(G)$ (cf. e.g. [19]). From the direct analogue of [44, Lemma 4.5.1] it is easy to see that

$$
\operatorname{Comm}(G)=\operatorname{Comm}\left(\mathrm{PSL}_{2}(\mathbb{Z})\right) \simeq \mathrm{PGL}_{2}(\mathbb{Q}) .
$$


For integers $l$ and $m$ we define the the map $B(l, m)$, generalizing $B(d)=B(d, 1)$ by

$$
B(l, m)=\left(\begin{array}{ll}
l & 0 \\
0 & m
\end{array}\right) \in \mathrm{PGL}_{2}(\mathbb{Q}) .
$$

It follows from [44, Lemma 4.5.2] that if $\alpha \in \mathrm{PGL}_{2}(\mathbb{Q})$ then there is an equality of sets

$$
\mathrm{PSL}_{2}(\mathbb{Z}) \alpha \mathrm{PSL}_{2}(\mathbb{Z})=\mathrm{PSL}_{2}(\mathbb{Z}) B(l, m) \mathrm{PSL}_{2}(\mathbb{Z}),
$$

where the integers $m \mid l$ are unique, depending on $\alpha$. Note that $l m$ can be negative. The following Lemma is then immediate from the fact that $G \subseteq C(G)$ and $G^{\prime} \subseteq C\left(G^{\prime}\right)$ :

Lemma 19. There is a set of representatives of $\tilde{C}\left(G^{\prime}, G\right)$ of the form $W B(l, m) V^{-1}$ where $l$ and $m$ are integers, $m \mid l, W \in G^{\prime} \backslash \mathrm{PSL}_{2}(\mathbb{Z})$ and $V \in G \backslash \mathrm{PSL}_{2}(\mathbb{Z})$.

To further reduce the number of possible maps, from an infinite to a finite set we use the following lemma.

Lemma 20. Assume that $N$ is the largest integer such that $G \leqslant \Gamma_{0}(N)$. If $B(l, m) \in$ $C\left(G^{\prime}, G\right)$ with $m \mid l$ then $(l / m) \mid N$.

Proof. It is easy to verify that if $g \in G$ then

$$
B(l, m)\left(\begin{array}{ll}
a & b \\
c & d
\end{array}\right) B(l, m)^{-1}=\left(\begin{array}{cc}
a & l b / m \\
m c / l & d
\end{array}\right)
$$

and for this to belong to $G^{\prime}$ we need in particular that both $l b / m$ and $m c / l$ are integers. If $m \mid l$ then it follows that $G \leqslant \Gamma_{0}(l / m)$ and hence $l / m$ divides $N$.

The following corollary also covers the case of conjugate subgroups of $\Gamma_{0}(N)$.

Corollary 21. Assume that $N$ is the largest integer such that $G$ is conjugate to a subgroup of $\Gamma_{0}(N)$. If $A \in C\left(G^{\prime}, G\right)$ can be written $A=W B(l, m) V^{-1}$ with $W, V \in \mathrm{PSL}_{2}(\mathbb{Z})$ and $m \mid l$ then $(l / m) \mid N$.

Proof. Apply the previous Lemma to the conjugated groups $W G^{\prime} W^{-1}$ and $V G V^{-1}$.

It is now possible to give an explicit description of the set of possible maps which can generate oldforms.

Proposition 22. Let $N$ be the largest integer such that $G$ is conjugate to a subgroup of $\Gamma_{0}(N)$. Then a complete set of representatives of $\tilde{C}\left(G^{\prime}, G\right)$ is contained in the finite set

$$
\left\{W B(l, m) V^{-1}: m|l| N, W \in G^{\prime} \backslash \mathrm{PSL}_{2}(\mathbb{Z}) \text {, and } V \in G \backslash \mathrm{PSL}_{2}(\mathbb{Z})\right\} .
$$

The final computation to find all possible old form maps is then done by computing the above set and quotient out by $C\left(G^{\prime}\right)$ from the left and $C(G)$ from the right. Using this proposition we searched through the database and found examples of non-trivial $\tilde{C}\left(G^{\prime}, G\right)$ in 58 cases. The first few examples are displayed in Table 1 , where $N$ is the maximal $N$ such that $G$ is conjugate to a subgroup of $\Gamma_{0}(N)$ 


\begin{tabular}{|c|c|c|c|c|c|}
\hline \multicolumn{3}{|c|}{$G$} & \multicolumn{2}{|c|}{$G^{\prime}$} & \multirow[t]{2}{*}{$\tilde{C}\left(G^{\prime}, G\right)$} \\
\hline Signature & $\sigma_{T}$ & $N$ & Signature & $\sigma_{T}$ & \\
\hline$(9 ; 0,2,3,0)$ & $(134852)(679)$ & 1 & & & \\
\hline$(9 ; 0,3,1,0)$ & $(1752)(348)(69)$ & 2 & $(3 ; 0,1,3,0)$ & $(132)$ & \} \\
\hline & & & $\begin{array}{c}\mathrm{PSL}_{2}(\mathbb{Z}) \\
(3 ; 0,2,1,0)\end{array}$ & $(12)(3)$ & $\begin{array}{c}\left\{I, B(2) S T^{-1}\right\} \\
\left\{I, T B(2) S T^{-1}\right\}\end{array}$ \\
\hline$(9,0$ & $(101002)(0)$ & 2 & $\begin{array}{c}\mathrm{PSL}_{2}(\mathbb{Z}) \\
(3 ; 0,2,1,0)\end{array}$ & $(12)(3)$ & $\begin{array}{c}\left\{I, B(2) S T^{-1}\right\} \\
\left\{I, T B(2) S T^{-1}\right\}\end{array}$ \\
\hline
\end{tabular}

Table 1: Exampes of non-trivial conjugators for noncongruence subgroups.

\subsection{Symmetries acting on the space of Maass cusp forms}

It is easy to see that the reflection $J: z \mapsto-\bar{z}$ acts on $S$ and $R$ by conjugation as

$$
S^{*}=J S J=S, \quad \text { and } \quad R^{*}=J R J=S R^{-1} S=S R^{2} S .
$$

Hence, if $G$ is represented by the legitimate pair $\Sigma=\left(\sigma_{S}, \sigma_{R}\right)$ then $G^{*}=J G J$ corresponds to the legitimate pair

$$
\Sigma^{*}=\left(\sigma_{S}^{*}, \sigma_{R}^{*}\right)=\left(\sigma_{S}, \sigma_{S} \sigma_{R}^{2} \sigma_{S}\right) .
$$

Since $\mathrm{PGL}_{2}(\mathbb{Z}) / \mathrm{PSL}_{2}(\mathbb{Z}) \simeq\{1, J\}$ it is clear that the set of $\mathrm{PGL}_{2}(\mathbb{Z})$ conjugates of $G$ is

$$
\left\{A G A^{-1} \mid A \in \mathrm{PGL}_{2}(\mathbb{Z})\right\}=(G) \cup\left(G^{*}\right)
$$

where it may or may not happen that $\left(G^{*}\right)=(G)$, depending on whether $G^{*}$ is conjugate to $G$ or not, or in other words, whether $\Sigma^{*} \sim \Sigma$ or not. It follows that the number of $\mathrm{PGL}_{2}(\mathbb{Z})$ conjugates is either equal to the number of $\mathrm{PSL}_{2}(\mathbb{Z})$ conjugates or twice as many. In table 2 we set $h_{G}=1$ or 2 to indicate these different cases. It turns out that if $G$ and $G^{*}$ are conjugate in $\mathrm{PSL}_{2}(\mathbb{Z})$ this implies the existence of a symmetry which can potentially be used to prove the existence of (odd) Maass cusp forms on the group $G$.

Observe that if $f: \mathcal{H} \rightarrow \mathbb{C}$ is invariant under $G$ and $A \in \mathrm{PGL}_{2}(\mathbb{R})$ then $f_{\mid A}$ is invariant under the conjugate group $A^{-1} G A$. Since the Laplacian, $\Delta$, is invariant under the action of $\mathrm{PGL}_{2}(\mathbb{R})$ it is clear that the spectra of $G$ and $A^{-1} G A$ are equal. It is therefore sufficient to study a single representative in each $\mathrm{PGL}_{2}(\mathbb{Z})$ conjugacy class.

We will now give a precise statement about a sufficient condition that newforms exist on a subgroup of $\mathrm{PSL}_{2}(\mathbb{Z})$. The following theorem gives an explanation for all newforms on noncongruence and non-cycloidal subgroups which we have found.

Theorem 23. Let $G$ be a subgroup of $\mathrm{PSL}_{2}(\mathbb{Z})$ and assume that there exists a nontrivial element $\alpha \in C(G)$ such that $\alpha$ preserves the cusp classes of $G$ and $\alpha^{2}=\mathbb{1}_{2}$. Then $L^{2}(G \backslash \mathcal{H})$ splits into a direct sum of $\alpha$-eigenspaces corresponding to the eigenvalues 1 and -1 . Furthermore, the Eisenstein series all belong to the +1 eigenspace.

Proof. Extending $[68, \mathrm{I}, \mathrm{S} 1-2]$ to $\mathrm{PGL}_{2}(\mathbb{R})$ we see that $\alpha$ induces a modular correspondence. It is easy to see that the action of $\alpha$ is self-adjoint on $L^{2}(G \backslash \mathcal{H})$ and we therefore 
obtain a decomposition into $\alpha$-eigenspaces. Since the action of $\alpha$ is linear and $\alpha^{2}$ acts as the identity it is clear that the only possible eigenvalues are +1 and -1 . In other words, $L^{2}(G \backslash \mathcal{H})=L_{+}^{2}(G \backslash \mathcal{H}) \oplus L_{-}^{2}(G \backslash \mathcal{H})$ where

$$
L_{ \pm}^{2}(G \backslash \mathcal{H})=\left\{f \in L^{2}(G \backslash \mathcal{H}) \mid f_{\mid \alpha}= \pm f\right\} .
$$

In Section 3 we saw that $L^{2}(G \backslash \mathcal{H})$ has a spectral decomposition of the form $L^{2}(G \backslash \mathcal{H})=$ $\mathbb{C} \oplus \mathcal{M}^{0} \oplus \mathcal{M}^{\text {res }} \oplus \mathcal{E}$, where the continuous part, $\mathcal{E}$, is spanned by Eigenpackets corresponding to Eisenstein series, $\mathcal{M}^{0}$ is spanned by Maass cusp forms and $\mathcal{M}^{\text {res }}$ by non-constant residues of Eisenstein series. Since $\alpha$ preserves cusp classes it follows from [68, Lemma 2.6] extended to $\mathrm{PGL}_{2}(\mathbb{R})$, together with the fact that $\alpha^{2} \in G$, that $\mathbb{C} \oplus \mathcal{E} \oplus \mathcal{M}^{\text {res }} \subset L_{+}^{2}(G \backslash \mathcal{H})$. Therefore $L_{-}^{2}(G \backslash \mathcal{H}) \subset \mathcal{M}^{0}$, that is, $L_{-}^{2}(G \backslash \mathcal{H})$ is spanned by Maass cusp forms.

Proposition 24. Let $G$ be a noncongruence group and assume that there is an $\alpha$ as in Theorem 23. Let $G \leqslant G^{\prime}$ be a supergroup with $\alpha \in C\left(G^{\prime}\right)$ and assume that $\gamma \in C\left(G^{\prime}, G\right)$ is such that $\gamma \alpha \gamma^{-1} \in C\left(G^{\prime}\right)$. Then the orthogonal complement of $\gamma \mathcal{M}^{0}\left(G^{\prime}\right)$ in $\mathcal{M}^{0}(G)$ is infinite-dimensional.

Proof. Let $\left\{V_{j}\right\}_{j=1}^{n} \simeq G \backslash G^{\prime}$ be a set of right coset representatives and $D \subset \mathcal{H}$ a small disk chosen such that $\gamma^{-1} A(D) \cap D \neq \emptyset$ for all $A \in \mathrm{PGL}_{2}(\mathbb{Z}) \backslash\left\{\mathbb{1}_{2}\right\}$. That such a disk exists is easy to show using the fact that $\mathrm{PSL}_{2}(\mathbb{Z})$ acts properly discontinuous on $\mathcal{H}$.

Let $f_{0}:\{1, \ldots, n\} \rightarrow \mathbb{C}$ be any function with $\sum_{j=1}^{n} f_{0}(j)=0$ which is not identically zero and define $f: \mathcal{H} \rightarrow \mathbb{C}$ by

$$
f(z)= \begin{cases}f_{0}(j), & \text { if } z \in G \gamma^{-1} V_{j} D, \\ -f_{0}(j), & \text { if } z \in G \alpha \gamma^{-1} V_{j} D, \\ 0, & \text { otherwise. }\end{cases}
$$

Then it is easy to see that $f$ is $G$-invariant, not identically zero, and that $f \in L_{-}^{2}(G \backslash \mathcal{H})$. Furthermore, if $g \in \mathcal{M}^{0}\left(G^{\prime}\right)$ then

$$
\begin{aligned}
\left\langle f, g_{\mid \gamma}\right\rangle= & \sum_{j=1}^{n}\left(\int_{\gamma^{-1} V_{j} D}+\int_{\alpha \gamma^{-1} V_{j} D}\right) f \bar{g}_{\mid \gamma} d \mu=\sum_{j=1}^{n} f_{0}(j) \int_{V_{j} D} \bar{g} d \mu \\
& -\sum_{j=1}^{n} f_{0}(j) \int_{\alpha \gamma^{-1} V_{j} D} \bar{g} d \mu=\left(\int_{D} \bar{g} d \mu-\int_{\gamma \alpha \gamma^{-1} D} \bar{g} d \mu\right) \sum_{j=1}^{n} f_{0}(j)=0,
\end{aligned}
$$

where we used $\gamma \alpha \gamma^{-1} V_{j} \in G^{\prime} \gamma \alpha \gamma^{-1}$. It follows that $f$ is orthogonal to $g_{\mid \gamma}$ and from the spectral expansion of $f$ into a sum of Maass cusp forms it follows that all non-vanishing terms must also be orthogonal to $g_{\mid \gamma}$. Finally, by varying the set $D$ we see that there must be infinitely many linearly independent Maass cusp forms in the orthogonal complement of the space $\gamma \mathcal{M}^{0}\left(G^{\prime}\right)$.

While the previous Proposition gives a condition for a given orthogonal complement to be infinite-dimensional there is in general no guarantee that the intersection of two such spaces is infinitely dimensional. It is indeed not even clear whether the intersection of all the orthogonal complements for a given supergroup $G^{\prime}$ is infinite-dimensional in the case when $C\left(G^{\prime}, G\right)$ is non-trivial. The following Proposition gives one condition under which this is true (and this condition is satisfied by the first pair of groups in Table 1). 
Proposition 25. Let $G$ be a noncongruence group and assume that there is an $\alpha$ as in Theorem 23. Let $G \leqslant G^{\prime}$ be a supergroup that satisfies

(a) $\alpha \in C\left(G^{\prime}\right)$ and

(b) $C\left(G^{\prime}, G\right)=C\left(G^{\prime}\right)$ (in other words, all conjugators of $G$ in $G^{\prime}$ normalize $G^{\prime}$ ).

Then the orthogonal complement of the entire old space from $G^{\prime}$ :

$$
\bigcap_{\gamma \in C\left(G^{\prime}, G\right)}\left(\gamma \mathcal{M}^{0}\left(G^{\prime}\right)\right)^{\perp}
$$

in $\mathcal{M}^{0}(G)$ is infinite-dimensional.

Proof. With $f(z)$ as in the proof of Proposition 24 but with $\gamma=1$ we see that if $\delta \in$ $C\left(G^{\prime}, G^{\prime}\right)$ then

$$
\left\langle f, g_{\delta}\right\rangle=\sum_{i=1}^{n} f_{i}\left\{\int_{D} \overline{g\left(\delta V_{i} z\right)} d \mu(z)-\int_{D} \overline{g\left(\delta \alpha V_{i} z\right)} d \mu(z)\right\}=0
$$

where we used the fact that $\delta V_{i} \delta^{-1} \in G^{\prime}$ and $\delta \alpha V_{i} \alpha^{-1} \delta^{-1} \in G^{\prime}$.

In the cases where the last proposition applies we can now deal with the entire oldform space from a given supergroup. When there are intermediate subgroups, i.e. $G<G^{\prime}<$ $G^{\prime \prime} \leqslant \operatorname{PSL}_{2}(\mathbb{Z})$ then the situation is more delicate as it may happen that there are oldforms from $G^{\prime \prime}$ in $G$ which are not oldforms on $G^{\prime}$. It seems that the best result we can achieve with this approach is the following theorem:

Theorem 26. Let $G$ be a noncongruence subgroup and assume that there is an $\alpha$ as in Theorem 23. If there is precisely one maximal supergroup, $G^{\prime}$, which satisfy $\alpha \in C\left(G^{\prime}\right)$, $C\left(G^{\prime}, G\right)=C\left(G^{\prime}\right)$ and for all intermediate subgroups $G<G^{\prime}<G^{\prime \prime} \leqslant \mathrm{PSL}_{2}(\mathbb{Z})$ we have $C\left(G^{\prime \prime}, G\right)=C\left(G^{\prime \prime}, G^{\prime}\right)$. Then the space of new Maass cusp forms on $G, \mathcal{M}^{\text {new }}(G)$, is infinite-dimensional.

Proof. This follows directly from the previous proposition since $C\left(G^{\prime \prime}, G\right)=C\left(G^{\prime \prime}, G^{\prime}\right)$ implies that the component $\gamma \mathcal{M}^{0}\left(G^{\prime \prime}\right)$ of the old space sum is contained in $\mathcal{M}^{0}\left(G^{\prime}\right)$.

This theorem can be viewed as a strengthening of a theorem by Venkov [71, Thm. 11.1] in the special case of subgroups of the modular group. Venkov's theorem requires $n^{2}$ symmetries (generalized Hecke operators) if the number of cusps is $n$, while our theorem only requires a single symmetry, but instead require that this is an involution and preserves cusp classes. The above proof uses only elementary methods but is unable to yield quantitative results about distribution of eigenvalues. It should, however, be possible to apply the Selberg trace formula to the modular correspondence given by $\alpha$ (as in [68]) to obtain a Weyl's law for $L_{-}^{2}(G \backslash \mathcal{H})$ but we leave this approach for possible future work.

Using Theorem 26 together with the computation of subgroups, their symmetries and sets of oldform maps we conclude the following Theorem:

Theorem 27. If $G$ is a noncongruence subgroup of index at most 17, for which there exists a reflectional symmetry which preserve the cusp classes of $G$ then the space of new Maass cusp forms on $G$ is infinite-dimensional. 
Proof. We have verified the conditions of Theorem 26 for all subgroups up to and including index 17 where there is a corresponding cusp preserving reflectional symmetry.

Remark 28. To provide context of the above theorem, there are 2528 conjugacy classes of noncongruence subgroups of index at most 17 . Out of these, 330 possess a reflectional symmetry, of which only 28 do not preserve cusp classes. The theorem above therefore shows that there are 302 noncongruence subgroups of index at most 17 with an infinite number of new Maass cusp forms. Data up to index 11 is given in Table 2 and more data can be obtained from [67] or directly from the author.

To use Theorem 26 we must find a permutation $\tau$ which conjugates the pair $\left(\sigma_{S}, \sigma_{R}\right)$ into $\left(\sigma_{S}^{*}, \sigma_{R}^{*}\right)$. Then we must find an $A \in \mathrm{PSL}_{2}(\mathbb{Z})$ with $\phi(A)(1)=\tau(1)$ and set $\alpha=J A$. We then need to check if $\alpha^{2} \in G$, and if it is then we must also see how the various cusp classes transform under $\alpha$. To be certain that we actually prove existence of newforms we must also investigate the possible supergroups between $G$ and $\mathrm{PSL}_{2}(\mathbb{Z})$.

Remark 29. It is easy to see that if $\alpha \in \mathrm{PGL}_{2}(\mathbb{Z})$ corresponds to the permutation $\tau$ as above then $\alpha$ preserves the cusp classes of $G$ if and only if $\tau$ preserves the cycles of $\sigma_{T}$. Since conjugation with a permutation which fixes 1 preserves the group it is in fact sufficient to require that $\tau(1)$ belongs to either the same cycle as 1 or to another cycle of the same length as that containing 1 in $\sigma_{T}$.

There are two special cases, in which we can see the exact form of $\alpha$ and only have to investigate the permutation of the cusp classes and possible supergroups.

Example 30. One example which we can easily check for is if $G^{*}=S G S$, that is, if $\alpha=S J: z \mapsto \frac{1}{z}$ (a reflection in the unit circle). Since $\sigma_{R^{*}}=\sigma_{S} \sigma_{R}^{2} \sigma_{S}$ and $\sigma_{S}^{*}=\sigma_{S}$ it is clear that we can make this choice of $\alpha$ if and only if $\left(\sigma_{S}, \sigma_{R}\right) \sim_{1}\left(\sigma_{S}, \sigma_{R}^{2}\right)$.

Example 31. If $G^{*}=T^{-k} G T^{k}$ with $k \in \mathbb{Z}$ then we can take $\alpha$ as a reflection in the line $\Re z=\frac{k}{2}$, that is, $\alpha: z \mapsto k-\bar{z}$. It is clear that $\alpha^{2}=\mathbb{1}_{2} \in G$. Here we write $T^{k}$ for the map $z \mapsto z+k$ for any $k \in \mathbb{R}$ and observe that we can write $\alpha=T^{k} J$ where, as usual $J: z \mapsto-\bar{z}$.

The last example is of particular importance since, first of all, it occurs in several instances which we have checked, and second, it enables us to describe the Fourier expansions in more detail whenever such a symmetry is present.

\subsection{Reflection in a vertical line}

Let $G \leqslant \mathrm{PSL}_{2}(\mathbb{Z})$, assume that $G^{*}=T^{-k} G T^{k}$ for some $k \in \mathbb{Z}$ and set $\alpha=T^{k} J$. Let $h_{1}$ denote the width of the cusp $\infty$ and choose the cusp normalizing map $\sigma_{1}: z \mapsto h_{1} z$. The Maass cusp form $f \in \mathcal{M}^{0}(G, R)$ now has the Fourier expansion at infinity

$$
f_{1}(z)=f\left(h_{1} z\right)=\sum_{n \neq 0} c(n) \kappa_{n}(y ; i R) e(n x),
$$


where we recall that $\kappa_{n}(y ; i R)$ is real. Applying the reflection $\alpha: z \mapsto k-\bar{z}$ we find that

$$
\begin{aligned}
(f \mid \alpha)_{1}(z)=f\left(k-h_{1} \bar{z}\right) & =\sum_{n \neq 0} c(n) \kappa_{n}(y ; i R) e\left(n\left(-x+k / h_{1}\right)\right) \\
& =\sum_{n \neq 0} c(-n) e\left(-n k / h_{1}\right) \kappa_{n}(y ; i R) e(n x) .
\end{aligned}
$$

If we then apply complex conjugation, $K: z \mapsto \bar{z}$, written $f \mid K(z):=\overline{f(z)}$, we see that

$$
(f|\alpha| K)_{1}(z):=\overline{f\left(k-h_{1} \bar{z}\right)}=\sum_{n \neq 0} \overline{c(n)} e\left(-n k / h_{1}\right) \kappa_{n}(y ; i R) e(2 \pi|n| y) e(n x) .
$$

There are thus two interesting involutions acting on $\mathcal{M}^{0}(G, R)$ : first $\alpha$ itself, which is linear, and then $\alpha K$ which is anti-linear. It follows that $\alpha$ has eigenvalues in $\{ \pm 1\}$ and all eigenvalues of $\alpha K$ lie un the unit circle. For now assume, without loss of generality, that $f$ is normalized so that $c(1)=1$. If $c(1)=0$ the arguments below can of course be used for any non-zero Fourier coefficient.

It is easy to see from the Fourier series above that if $f(\alpha z)=\epsilon f(z)$ with $\epsilon \in\{ \pm 1\}$ then $c(-n)=\epsilon c(n) e\left(n k / h_{1}\right)$ for all $n$, and in particular

$$
c(-1)=\epsilon e\left(k / h_{1}\right)=\epsilon e^{\frac{2 \pi i k}{h_{1}}} .
$$

The cases $\epsilon=1$ and -1 are usually referred to as even and odd (with respect to $\alpha$ ) in analogue with the usual situation for congruence subgroups where we can always take $\alpha=J$. It follows that we can determine whether a Maass form is even or odd from the Fourier coefficient $c(-1)$.

Furthermore, if $f|\alpha| K=\eta f$ then $|\eta|=1$ and if $c(n) \neq 0$ then

$$
\overline{c(n)} / c(n)=e^{-2 i \operatorname{Arg}(c(n))}=\eta e\left(n k / h_{1}\right),
$$

where Arg denotes the principal branch of the argument. Setting $n=1$ and using that $c(1)=1$ we see immediately that $\eta=e\left(-k / h_{1}\right)$ and thus, for any $n$ with $c(n) \neq 0$

$$
\operatorname{Arg}(c(n))=\pi\left(j+(1-n) k / h_{1}\right),
$$

for some integer $j$. This means in particular that $a(n)=c(n) e\left((n-1) /\left(2 h_{1}\right)\right)$ is a real number for all $n$. See, for instance, Figure 6 where plotted the real numbers $a(n)=c(n) e((n-1) / 16)$ for $n$ in different congruence classes modulo 8 .

If we know that there is a reflectional symmetry of the form indicated above we can use this fact to reduce the computations of Maass cusp forms with general (complex) Fourier expansions to computations where the unknowns are all real numbers. Another possibility is to use these relations as additional checks that our computations are correct. This is illustrated in Tables 3 to 6 , where the relation $c(-1)= \pm e^{\frac{2 \pi i k}{h_{1}}}$ is used to provide an additional indication of the error.

In Table 2 we indicate those subgroups which have a symmetry of the form $\alpha$ : $z \mapsto k-\bar{z}$ which preserves cusp classes by giving the value of $k$ in the column $k_{\alpha}$. Although we also found other types of symmetries, for instance of the form $G^{*}=A G A^{-1}$ 
where $A$ is not of the form $T^{k}$, these only appeared in cases where we also had a simpler reflectional symmetry. Observe that even though both cycloidal and congruence subgroups have new Maass cuspforms, they do not necessarily possess a reflectional symmetry. See for instance the group of signature $(8 ; 0,1,2,2)$ given by the permutations $\sigma_{S}=(1)(2)\left(\begin{array}{ll}3 & 4\end{array}\right)\left(\begin{array}{lll}5 & 6\end{array}\right)\left(\begin{array}{ll}7 & 8\end{array}\right)$ and $\sigma_{R}=\left(\begin{array}{llll}1 & 4 & 7\end{array}\right)\left(\begin{array}{lll}2 & 6 & 8\end{array}\right)(3)(5)$, which noes not have any reflectional symmetry.

\subsection{Explicit examples of subgroups with symmetries}

In this section we will give explicit examples of subgroups where there is a symmetry of the form $\alpha: z \mapsto k-\bar{z}$, in other words, where $G^{*}=J G J=T^{-k} G T^{k}$ for some integer $k$. Cf. e.g. Example 31. As an illustration of the results we are using we provide a few cases with complete details in how the symmetry acts and how it preserves the cusp classes (or not).

To make it easier to refer to the groups in Table 2 we let $\Gamma\left(h ; g, \kappa, e_{2}, e_{3} ; N, i\right)$ denote the subgroup of signature $\left(h ; g, \kappa, e_{2}, e_{3}\right)$ and generalized level $N$ given by the $i$ th matching entry in the table. This specification does indeed determine the group uniquely (and independently of our table) since the conjugacy classes are ordered according to their representatives, which are in turn ordered lexicographically in terms of the permutation $\sigma_{R}$. More precisely the ordering is in terms of the representation of $\sigma_{R}$ as a vector in $\mathbb{Z}^{h}$, for instance $\sigma=(125)(3)(4) \sim(2,5,3,4,1)$ and $\tau=(123)(45)$ corresponds to $(2,3,1,5,4)$ which implies that $\tau<\sigma$ with respect to this ordering. However, observe that the labelling clearly does depend on knowing all conjugacy classes of the given signature.

To our knowledge, the only way to obtain a unique label which is independent of knowing any other groups is to use the complete specification of the group, for instance by giving both the permutations $\sigma_{S}$ and $\sigma_{R}$. Alternatively, since we always fix the permutation $\sigma_{S}$ it is sufficient to specify the signature or $e_{2}$, together with $\sigma_{T}$.

Example 32. Consider the group $G=\Gamma(10 ; 0,2,2,1 ; 8,1)$ given by the permutations

$$
\sigma_{S}=(1)(2)\left(\begin{array}{ll}
3 & 4
\end{array}\right)\left(\begin{array}{ll}
5 & 6
\end{array}\right)\left(\begin{array}{l}
7 \\
8
\end{array}\right)\left(\begin{array}{ll}
9 & 10
\end{array}\right) \quad \text { and } \quad \sigma_{R}=\left(\begin{array}{lll}
1 & 2 & 5
\end{array}\right)(3)\left(\begin{array}{lll}
4 & 7 & 9
\end{array}\right)\left(\begin{array}{lll}
6 & 10 & 8
\end{array}\right)
$$

and let $h: \mathrm{PSL}_{2}(\mathbb{Z}) \rightarrow S_{10}$ be the homomorphism defined by $\left(\sigma_{S}, \sigma_{R}\right)$. Then

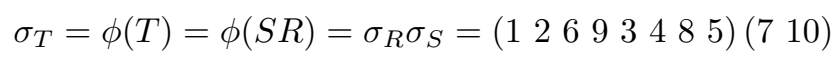

and therefore the generalized level of this group is 8. It easy to see that $\sigma_{R^{*}}=\sigma_{S} \sigma_{R}^{2} \sigma_{S}=$

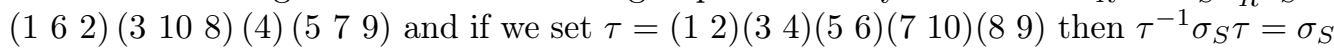
and $\tau^{-1} \sigma_{R} \tau=\sigma_{R^{*}}$. Hence, by Theorem 9 it follows that $G^{*}$ and $G$ are conjugate in $\operatorname{PSL}_{2}(\mathbb{Z})$. Since $\tau(1)=\sigma_{T}(1)=2$ we conclude with the help of Theorem 8 that $G^{*}=T^{-1} G T$ or in other words $T J G J T^{-1}=G$. As the associated symmetry we can choose $\alpha=T J: z \mapsto 1-\bar{z}$, which is a reflection in the line $x=\frac{1}{2}$. The cusp representatives are $p_{1}=\infty$ and $p_{2}=3$. Now $\alpha(\infty)=\infty$ and $\alpha\left(p_{2}\right)=-2$. It is easy to verify that the matrix

$$
B=\left(\begin{array}{cc}
-3 & 11 \\
1 & -4
\end{array}\right)=T^{-2} S T S T^{-3}
$$

belongs to $G$ by computing the associated permutation

$$
\begin{gathered}
h(B)=\sigma_{T}^{-2} \sigma_{S} \sigma_{T} \sigma_{S} \sigma_{T}^{-3}=(1)\left(\begin{array}{lll}
2 & 5 & 7
\end{array}\right)\left(\begin{array}{lll}
3 & 9 & 4
\end{array}\right)\left(\begin{array}{lll}
6 & 10 & 8
\end{array}\right), \\
27
\end{gathered}
$$


which clearly fixes 1 . Furthermore $B(3)=-2$ so 3 and -2 belong to the same cusp class and hence $\alpha$ preserves cusp classes. As mentioned in Remark 29 above, we can in fact see this directly from the permutation $\tau$ since it preserves the two cycles of $\sigma_{T}$. In this case any $\alpha$-odd newform $f$ on $G$ must have $c(-1)=-e^{\frac{2 \pi i}{8}}$, which is consistent with our experimental findings. See e.g. Table 6 .

Example 33. Consider the group $G=\Gamma(10 ; 0,3,0,1 ; 20,1)$ given by the permutations

$$
\sigma_{S}=\left(\begin{array}{lll}
1 & 2
\end{array}\right)\left(\begin{array}{lll}
3 & 4
\end{array}\right)\left(\begin{array}{lll}
5 & 6
\end{array}\right)(78)(910), \quad \text { and } \quad \sigma_{R}=(1)\left(\begin{array}{llll}
2 & 3 & 5
\end{array}\right)\left(\begin{array}{llll}
4 & 6 & 7
\end{array}\right)\left(\begin{array}{lll}
8 & 9 & 10
\end{array}\right)
$$

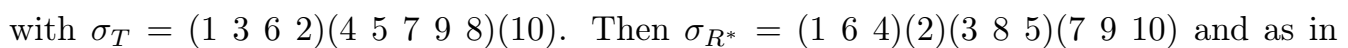
the previous example we see that if $\tau=\left(\begin{array}{lll}1 & 2\end{array}\right)\left(\begin{array}{l}6 \\ 6\end{array}\right)\left(\begin{array}{ll}5 & 4\end{array}\right)(78)(9)(10)$ then $\tau^{-1} \sigma_{S} \tau=\sigma_{S}$ and $\tau^{-1} \sigma_{R^{*}} \tau=\sigma_{R}$. Since $\tau(1)=\sigma_{T}^{3}(1)=2$ we conclude that $G^{*}=T^{-3} G T^{3}$ and hence $G$ has a symmetry of the form $\alpha: z \mapsto 3-\bar{z}$, a reflection in the line $x=3 / 2$. Since $\tau$ preserves the cycles of $\sigma_{T}$ it follows that $\alpha$ preserves all cusp classes (this can of course be verified directly as in the previous example). In this case an $\alpha$-odd newform $f$ on $G$ must have $c(-1)=-e^{\frac{3 \pi i}{4}}$, which agrees with the experiments See e.g. Table 6 .

Example 34. Now, as an example of a group without any newforms (according to the computations) consider the group $G=\Gamma(10 ; 0,3,0,1 ; 8,1)$ given by

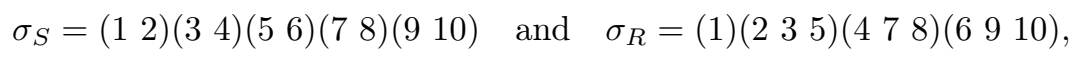

with $\sigma_{T}=(13745962)(8)(10)$. Then $\sigma_{R}^{*}=(164)(2)(378)(5910)$ and it is easy to check that $\tau=(12)(63)(54)$ (79) (8 10) conjugates the pairs $\left(\sigma_{S}, \sigma_{R}\right)$ and $\left(\sigma_{S}, \sigma_{R^{*}}\right)$, and hence that $G$ and $G^{*}$ are indeed $\mathrm{PSL}_{2}(\mathbb{Z})$ conjugate. More precisely $G^{*}=T^{-7} G T^{7}$. The corresponding symmetry we obtain is $\alpha: z \mapsto 7-\bar{z}$.

As cusp representatives for $G$ we can choose $p_{1}=\infty, p_{2}=2$ and $p_{3}=-3$. Since $\alpha(2)=5=-3+8$ and $T^{8} \in G$ it follows that $\alpha$ permutes the cusp classes of $p_{2}$ and $p_{3}$. This can of course be seen directly since $\tau$ interchanges the cycles (8) and (10) in $\sigma_{T}$.

In this case, using the same algorithm as for the other groups, we found no Maass cusp forms with spectral parameters up to $R=20$, except for oldforms from $\mathrm{PSL}_{2}(\mathbb{Z})$.

Example 35. Consider the cycloidal group $G=\Gamma(10 ; 1,1,0,1 ; 10,1)$ given by

$$
\sigma_{S}=\left(\begin{array}{ll}
1 & 2
\end{array}\right)\left(\begin{array}{ll}
3 & 4
\end{array}\right)(56)(78)(910) \quad \text { and } \quad \sigma_{R}=(1)(235)(479)\left(\begin{array}{lll}
6 & 8 & 10
\end{array}\right),
$$

with $\sigma_{T}=(13710458962)$. The permutation $\tau=(12)(36)(45)(79)(810)$ conjugates $\left(\sigma_{S}, \sigma_{R}\right)$ and $\left(\sigma_{S}, \sigma_{R^{*}}\right)$. Hence $G^{*}=T^{-9} G T^{9}$ and we can take the symmetry $\alpha: z \mapsto-\bar{z}+9$, which clearly also preserves the cusp. Any $\alpha$-odd function $f$ on $G$ will have Fourier coefficient $c(-1)=-e^{\frac{9 \pi i}{10}}$, and any $\alpha$-even function will have $c(-1)=e^{\frac{9 \pi i}{10}}$. Experimentally we found both odd and even newforms on this group! Cf. Table 5.

Example 36. As example of a group without reflectional symmetry consider $G=$ $\Gamma(7 ; 0,1,3,1)$ given by

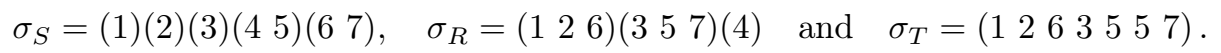

We have $\sigma_{R^{*}}=\left(\begin{array}{lll}1 & 7 & 2\end{array}\right)\left(\begin{array}{lll}3 & 6 & 4\end{array}\right)(5)$ and if $\tau \in S_{7}$ conjugates $\left(\sigma_{S}, \sigma_{R}\right)$ into $\left(\sigma_{S}, \sigma_{R^{*}}\right)$ then $\tau$ preserves the cycles of $\sigma_{S}$ and in particular it must permute the 1-cycles (1), (2), (3) 
and the 2-cycles $(45)$ and (6 7), each amongst themselves. Since it must also map 4 to 5 it follows that $\tau=\tau^{\prime}(45)(67)$ where $\tau^{\prime} \in S_{3}$. Since $\tau(6)=7$ it follows that $\tau$ must map the 3-cycle $\left(\begin{array}{lll}1 & 3 & 6\end{array}\right)$ to $\left(\begin{array}{lll}1 & 7 & 2\end{array}\right)$ and hence $\tau(1)=2, \tau(2)=1$ and thus $\tau(3)=3$ so that $\tau=\left(\begin{array}{ll}1 & 2\end{array}\right)(3)\left(\begin{array}{ll}4 & 5\end{array}\right)\left(\begin{array}{l}6 \\ 6\end{array}\right)$. However, it is now clear that $\tau$ maps the cycle $\left(\begin{array}{lll}3 & 5 & 7\end{array}\right)$ to $\left(\begin{array}{lll}3 & 4\end{array}\right)$ and not $\left(\begin{array}{ll}3 & 6\end{array}\right)$. It follows that $G$ and $G^{*}$ are not conjugate.

\section{Notes on the Algorithms}

Since one of the main goals is a systematic investigation of discrete eigenvalues for noncongruence subgroups of the modular group we must first construct a table of such groups. The number of congruence subgroups is asymptotically much smaller than the number of noncongruence groups but despite their abundance no systematic tabulation of them has appeared in the literature. In contrast, for congruence subgroups, there exist extensive tables, for instance by Cummins and Pauli [13].

\subsection{Algorithms for tabulating subgroups}

Since the spectrum of the Laplacian is invariant under conjugation by $\mathrm{PGL}_{2}(\mathbb{Z})$ we only need to find Maass forms for one representative per conjugacy class.

Let $h$ be a positive integer. We know from Theorem 8 that subgroups of index $h$ in $\mathrm{PSL}_{2}(\mathbb{Z})$ (up to conjugation) correspond to equivalence classes of legitimate pairs $\left(\sigma_{S}, \sigma_{R}\right)$ in $\mathrm{S}_{h}$.

From a computational point of view the hardest part is to first filter the list of all legitimate pairs into representatives modulo 1 and then into $\mathrm{S}_{h}$-conjugacy classes. It is well-known that conjugacy in $S_{h}$ is simply determined by the cycle structure but since we need to conjugate both $\sigma_{S}$ and $\sigma_{R}$ the problem is much more delicate. To simplify the first problem of finding representatives modulo 1 , in other words, we want a unique permutation per group, we first of all fix the permutation $\sigma_{S}$ uniquely. More precisely, we always assume that $\sigma_{S}=(1) \cdots\left(e_{2}\right)\left(e_{2}+1 e_{2}+2\right) \cdots(h-1 h)$ where $e_{2}$ is the number of fixed points of $\sigma_{S}$. Let $\mathrm{S}_{h, \sigma_{S}}$ denote the stabilizer of $\sigma_{R}$ in $\mathrm{S}_{h}$. Given such a $\sigma_{S}$ we then choose $\sigma_{R}$ such that it is the lexicographically smallest possible permutation in $\mathrm{S}_{h, \sigma_{S}}$, which is of order 3 , has $e_{3}$ fixed points, and such that $\sigma_{S}$ and $\sigma_{R}$ acts transitively on $\{1,2, \ldots, h\}$. To a large extent these choices can be made explicitly and we can use this to obtain a single example of a subgroups with a given signature. For $h=96$ this takes a few days on an Intel Xeon $2 \mathrm{GHz}$.

The second step involves taking the list of all pairs corresponding to a given signature and see which of these are conjugate to each other. This is much more time consuming and except for specifying that e.g. 3-cycles maps to 3-cycles and 1-cycles to 1-cycles it seems hard to avoid to iterate over (most of) $\mathrm{S}_{h, \sigma_{S}}$

To generate all elements of $S_{h}$ is relatively fast for modestly large $h$ (say $h \leq 17$ ), and there exist a number of fast algorithms to choose from, cf., e.g. [27]. However, except for a few signatures of index 18 we have only been able to completely partition subgroups into conjugacy classes up to index 17. Given a list of non-conjugated legitimate pairs we use Hsu's theorem to identify congruence subgroups. 


\section{On the computation of Maass forms for general subgroups}

The algorithm which we use to compute Maass forms and Eisenstein series was originally developed by Dennis A. Hejhal (based on an idea of Harold Stark) for computing Maass cusp forms on Hecke triangle groups [22, 23]. Subsequently this algorithm was adapted by the author to other Fuchsian groups, non-trivial weights and multiplier systems and even harmonic weak Maass forms [12, 64, 65]. It was also generalized by Helen Avelin to compute Eisenstein series and Green's functions [5, 6]. In another direction, Holger Then adapted the algorithm in a very efficient manner to enable computation of Maass cusp forms with large eigenvalues for Fuchsian groups with one cusp [29, 69]. Even though the algorithm is described in several of the above cited sources we will nevertheless include a comprehensive overview here for the sake of completeness and because there are some delicate points that needs to be stressed.

\subsection{Background}

Let $G$ be a subgroup of index $h$ in $\operatorname{PSL}_{2}(\mathbb{Z})$ and assume that all notation, unless otherwise mentioned, is as in Sections 2 and 3. We assume for simplicity that the coset representatives $\left\{V_{i}\right\} \simeq G \backslash \mathrm{PSL}_{2}(\mathbb{Z})$ are chosen so that the fundamental domain $\mathcal{F}_{G}=\cup V_{i} \mathcal{F}_{1}$ has vertices exactly at the cusp representatives $p_{1}, \ldots, p_{v_{\infty}}$. For the cusp $p_{j}$ with width $h_{j}$ we choose the cusp normalizer $\mathcal{N}_{j}$ with $\mathcal{N}_{j}(\infty)=p_{j}$ of the form $\mathcal{N}_{j}=A_{j} \rho_{j}$ with $A_{j} \in \mathrm{PSL}_{2}(\mathbb{Z}), \rho_{j}: z \mapsto h_{j} z$ and $\mathcal{N}_{j}^{-1} S_{j}=T$ where $S_{j}$ is a generator of $G_{p_{j}}$, the stabilizer of $p_{j}$. Let $h_{\max }$ denote the maximum cusp width and set $Y_{0}=\sqrt{3} /\left(2 h_{\max }\right)$.

\subsection{Setting up the system of equations}

Let $f \in \mathcal{M}^{0}(G, R)$ be a Maass cusp form for $G$ with spectral parameter $R$. The Fourier expansion of $f$ at the cusp $p_{j}$ is now of the form

$$
f_{j}(z)=f\left(\mathcal{N}_{j} z\right)=\sum_{n \neq 0} c_{j}(n) \kappa_{n}(y ; i R) e(n x)
$$

which in particular has period 1 . Let $\varepsilon>0$ be fixed and define a function $M: \mathbb{R}^{+} \rightarrow \mathbb{N}$ such that for all indices $j$ and all $z$ with $\Im(z) \geq Y$ we have the approximation

$$
f_{j}(z)=\widehat{f}_{j}(z)+\llbracket \varepsilon \rrbracket
$$

where we write $\llbracket \varepsilon \rrbracket$ for an unknown quantity absolutely bounded by $\varepsilon$ and let

$$
\widehat{f}_{j}(z)=\sum_{0<|n| \leq M(Y)} c_{j}(n) \kappa_{n}(y ; i R) e(n x) .
$$

That such an $M$ exists follows from the rapid decay of the $K$-Bessel function and the fact that the Fourier coefficients are asymptotically bounded by $c_{j}(n)=O(\sqrt{n})$ with an implied constant depending on $R$ and $G$. We define the function $I: \mathcal{H} \rightarrow\left\{1, \ldots, v_{\infty}\right\}$ by $I(z)=k$ where $k$ is the smallest integer with $\Im\left(\mathcal{N}_{k}^{-1} z\right)=\max _{j} \Im\left(\mathcal{N}_{j}^{-1} z\right)$.

Let $M_{0}=M\left(Y_{0}\right)$, choose $Q>M_{0}+10$ and $Y<Y_{0}$ and consider the set $\left\{z_{m}\right\}_{1-Q \leq m \leq Q}$ of equally spaced points along a horocycle at height $Y$, where $z_{m}=x_{m}+i Y$ with 
$x_{m}=(m-1 / 2) /(2 Q)$. Viewing the truncated series $\widehat{f}_{j}$ as a discrete Fourier transform and applying Fourier inversion over the horocycle we immediately find that

$$
c_{j}(n) \kappa_{n}(Y ; i R)=\frac{1}{2 Q} \sum_{1-Q}^{Q} f_{j}\left(z_{m}\right) e\left(-n x_{m}\right)+\llbracket \varepsilon \rrbracket .
$$

By using the $G$-invariance of $f$ we can transform this into a non-trivial linear system of equations for the Fourier coefficients. Let $w_{m, j}=\gamma_{j, m} \mathcal{N}_{j} z_{m} \in \mathcal{F}_{G}$ be the pullback of $\mathcal{N}_{j} z_{m}$ into the fundamental domain with $\gamma_{j, m} \in G$ and set $z_{m, j}^{*}=\mathcal{N}_{I(m, j)}^{-1} w_{m, j}=x_{m, j}^{*}+$ $i y_{m, j}^{*}$, where $I(m, j)=I\left(w_{m, j}\right)$. It is then immediate to check that $f_{j}\left(z_{m}\right)=f_{I(m, j)}\left(z_{m, j}^{*}\right)$ and since $y_{m, j}^{*} \geq Y_{0}>Y$ it follows that

$$
c_{j}(n) \kappa_{n}(Y ; i R)=\frac{1}{2 Q} \sum_{1-Q}^{Q} \widehat{f}_{j}\left(z_{m, j}^{*}\right) e\left(-n x_{m}\right)+\llbracket 2 \varepsilon \rrbracket .
$$

Inserting the Fourier series for $\widehat{f}_{j}\left(z_{m, j}^{*}\right)$ and subtracting the right hand side we obtain an "approximately homogeneous" linear system of equations

$$
\sum_{i=1}^{v_{\infty}} \sum_{0<|l| \leq M(Y)} c_{i}(l) V_{n l}^{j i}=\llbracket 2 \varepsilon \rrbracket \quad\left(1 \leq j \leq v_{\infty}, 0<|n| \leq M(Y)\right),
$$

where the matrix coefficients are given by

$$
V_{n l}^{j i}=\frac{1}{2 Q} \sum_{I(m, j)=i} \kappa_{l}\left(y_{m, j}^{*} ; i R\right) e\left(l x_{m, j}^{*}-n x_{m}\right)-\delta_{i j} \delta_{n l} \kappa_{n}(Y ; i R) .
$$

If the parameters $Y$ and $M$ are appropriately chosen then the system (19) turns out to be well-conditioned. The choice of parameters is a very delicate process which is best performed using an initial theoretical choice combined with automated adjustments. Initial choices given by the discussions in e.g. [29] and [23] applies here as well, after obvious modifications. One of the key observations is that the $K$-Bessel function $K_{i R}(x)$ is oscillating in the region $x<R$ and monotonic decreasing for $x>R$ (see e.g. [14, Vol II, pp. 87-88]). A good set of initial parameters involves choosing $M_{0}$ such that $2 \pi M_{0} Y_{0}>\max (R, 1)$ and $\left|K_{i R}\left(2 \pi Y_{0} M_{0}\right)\right|<\varepsilon \min \left(1, K_{i R}(R)\right)$. To ensure that the system is well-conditioned we would also like to choose $Y$ such that $K_{i R}(2 \pi Y n)$ is not too small for $1 \leq n \leq M_{0}$. Although it is difficult to predict exactly which parameters will give a well-conditioned system the numerical experiments indicate that it is only necessary to adjust the values of $Y$ and $M_{0}$ a small number of times. A robust algorithm for choosing good parameters is given for instance by Algorithm 12 in [29]. To demonstrate how the choice of $Y$ affects the conditioning of the system and and the need to decrease $Y$ if a larger number of coefficients are needed see the following example.

Example 37. Consider the full modular group and $R=12.1730083246796778$. With $M_{0}=22$ and $Y=0.866$ the matrix $V$ has condition number $c(V)=1432$ and with $Y=0.13$ the condition number is instead $c(V)=114$. In terms of the solution vectors we obtain the tentative Fourier coefficients $c(21)=316.26+7.91 i$ in the first case and 
$c(21)=-0.538592697732250-3.7 \cdot 10^{-16} i$ in the second. In the latter case the coefficient values are also found to satisfy a multiplicative relation $|c(21)-c(7) c(3)|<5 \cdot 10^{-14}$ while, in the first case $|c(21)-c(7) c(3)|>316$.

A further complication which appear in the current setting is the we need to make sure that the pullbacks involve all generators of the group, ideally in similar proportions. In the case of the modular group and Hecke triangle groups this is always the case as long as $Y<Y_{0}$ but in general the situation is less clear. For a condition which is easy to check numerically (a posteriori) see Section 8.6.

By arranging all the coefficients $c_{j}(n)$ into a vector, $C$, and the $V_{n l}^{i j}$ into a matrix $V:=V(R, Y)$ we see that the coefficients of the Maass cusp form $f$ satisfy an equation of the form

$$
V C=\llbracket 2 \varepsilon \rrbracket .
$$

\subsection{Normalizing the linear system}

The right hand side of (20) is (of course) unknown and we must instead solve the corresponding homogeneous system $V C=0$. Since the matrix $V$ will generally have full rank if computed to sufficiently high precision it is necessary to make some further assumptions to ensure the existence of non-trivial solutions. One such assumption, which is immediately satisfied by newforms on congruence subgroups, is to assume that $c_{1}(1)=$ 1. This leads to an inhomogeneous equation

$$
V^{\prime} C=-c_{1}(1) B^{\prime}=-B^{\prime}
$$

where $B^{\prime}$ is the column of $V$ corresponding to $c_{1}(1)$ and $V^{\prime}$ is simply $V$ with this column removed. We also remove a row from both $V^{\prime}$ and $B^{\prime}$ to ensure that the coefficient matrix of (21) is square. Experimentally we have found that the assumption $c_{1}(1)=1$ works in all cases when the multiplicity of the corresponding Laplace eigenvalue is 1 .

\subsection{Multiple eigenvalues.}

In case the multiplicity is greater than 1 it is also possible to prescribe more coefficients. For example, in the case of an oldform on $\Gamma_{0}(2)$ where we know that the multiplicity is 2 (assuming that the multiplicity is one on $\operatorname{PSL}_{2}(\mathbb{Z})$ ) we can obtain a basis by using the two normalizations $\left\{c_{1}(1)=1, c_{1}(2)=0\right\}$ and $\left\{c_{1}(1)=0, c_{1}(2)=1\right\}$. In this manner it is possible to use the same methods to locate eigenvalues of higher multiplicity. Note that $c_{1}(2)=0$ for a newform on $\Gamma_{0}(4)$ and in general some care has to be taken to choose appropriate coefficients. When searching for eigenvalues for non-congruence subgroups we usually use this method (with varying sets of Fourier coefficients) to check for eigenvalues of multiplicity up to at least 3 .

There is of course another natural approach to investigate existence of possibly multiple eigenvalues, namely to use the singular value decomposition of the matrix $V$. Since we are working with numerical approximations a more appropriate measure of the dimension of the kernel is given by the singular value decomposition. We can, for instance, count the number of singular values less than some given small positive $\delta$. This will additionally give us an idea of what the multiplicity of the corresponding Laplace eigenvalue should be. 
Example 38. Consider the spectral parameter $R=13.7797513518907$ which corresponds to an even Maass cusp form for the full modular group (this is correct to all decimals given as verified by Andreas Strömbergsson using the methods of [11]). Setting $M_{0}=10$ and $Y_{0}=0.86$ and applying the algorithm to the modular group we obtain a $21 \times 21$ matrix $\widetilde{V}\left(R, Y_{0}\right)$. Note that this matrix also contains a row and a column which are identically zero, corresponding to the constant coefficient. This is included in the implementation to make it easier to adapt the algorithms to Eisenstein series and weak Maass forms. The first few singular values, listed in order of increasing magnitude are $s_{1}=0, s_{2}=2 \cdot 10^{-14}$ and $s_{3}=2 \cdot 10^{-5}$. The same setup, but for $\Gamma_{0}(2)$, results in a $42 \times 42$ matrix with singular values $s_{1}=s_{2}=0, s_{3}=1 \cdot 10^{-14}, s_{4}=2 \cdot 10^{-14}$ and $s_{5}=4 \cdot 10^{-2}$.

\subsection{Identifying genuine spectral parameters.}

It is still the case that if $C$ is a vector of coefficients for a Maass cusp form with spectral parameter $R$ such that $c_{1}(1)=1$ then $C$ (without $c_{1}(1)$ ) will satisfy (21) up to an error of magnitue $2 \varepsilon$ but the converse is clearly not true since (21) will (in general) have a unique solution for any choice of parameters $R$ and $Y$. One of the main problems in this area of research is to identify which (if any) such solutions correspond to genuine Maass cusp forms. In other words, given a tentative set of parameters $Y, R$ and a solution $C$ to $(21)$ we would like to assert whether or not $R$ is close to a true spectral parameter and the solution vector is close to the corresponding Fourier coefficients.

In certain cases it is indeed possible to give a rigorous qualitative answer to this question. An algorithm for this was proposed and implemented by Booker, Strömbergsson and Venkatesh for the full modular group [11]. There are two major obstacles for extending this method to other groups. The first, and most important for our setting, is that it relies on the existence of Hecke operators for eliminating the continuous spectrum. The second obstacle is that it is necessary to write down explicit estimates for Taylor series along arcs corresponding to generators. While this is in principle possible to do for any given subgroup of the modular group it is a tedious process which seems to be difficult to automate.

An alternative approach for certifying the spectrum of hyperbolic surfaces was developed by Strohmaier and Uski [62]. Unfortunately their method only applies to compact surfaces and it is not immediately clear how to adapt it to more general surfaces with cusps. While it might be possible to incorporate parts of their algorithm into the framework of [11] to alleviate the burden of writing down the explicit estimates it would still be necessary to deal with the continuous spectrum.

The above can be summarized as saying that as far as the author is aware, there are currently no methods for rigorously verifying discrete eigenvalues of the Laplacian in the presence of continuous spectrum unless that spectrum can be described explicitly and isolated by, for instance, using Hecke operators.

Since rigorous arguments are not available we instead use a number of heuristic tests to determine whether a given solution to (20) corresponds to a genuine Maass cusp form or not. This problem is discussed in detail in most articles where this method is used to compute Maass cusp forms, see for instance [22, 23, 29, 65, 69]. One potential method, which has been employed in the computations discussed here is the following. For a given $R>0$ choose two different values $Y_{1}$ and $Y_{2}$ both less than $Y_{0}$. Then compute the two 
non-zero solution vectors $C\left(Y_{1}, R\right)=\left\{c_{i}(n)\right\}$ and $C^{\prime}=C\left(Y_{2}, R\right)=\left\{c_{i}^{\prime}(n)\right\}$ and set

$$
\phi(R)=\sum_{j \in J} \epsilon_{j}\left|c_{1}(j)-c_{1}^{\prime}(j)\right|
$$

for some index set $J$ (for instance $J=\{2,3,4\}$ ) where $\epsilon_{j} \in\{ \pm 1\}$ are suitably chosen. It follows that $\phi(R)$ will be close to zero if $R$ is close to a genuine spectral parameter and furthermore, if the signs $\epsilon_{j}$ are chosen appropriately then $\phi(R)$ will change sign close to true eigenvalues. It is thus possible to use, for instance, the secant method to locate zeros of $h$ and hence find a set of potential eigenvalues in an interval. Experimentally we find that using a single pair $Y_{1}$ and $Y_{2}$ still result in sporadic solutions. It is therefore necessary to use at least one more heuristic test, for instance using another pair of $Y_{i}$ s or one of the following:

(a) Compare Fourier expansions at different cusps. For congruence subgroups AtkinLehner theory implies that we can assume that there exists a basis which satisfy $c_{2}(n)= \pm c_{1}(n)$ (assuming $p_{2}=0$ ). For certain noncongruence subgroups there are similar relations, which can be expressed as $c_{2}(n) / c_{2}(1)= \pm c_{1}(n)$ for some particular choices of $n$ s.

(b) If the group has a reflectional symmetry of the type discussed in Section 6.3 then we can compare $c_{1}(-1)$ with the expression given by (15).

(c) An extremely reliable test which, unfortunately, only applies to congruence subgroups is to check multiplicativity of Fourier coefficients coming from Hecke operators, e.g. $c_{1}(2) c_{1}(3)=c_{1}(6)$.

(d) Verify that the function defined through the expansions (18) with the computed coefficients is (approximately) invariant under the generators of $G$.

Although tests of the form (b) involving only a single coefficient will result in many sporadic solutions it is a very useful measure of the potential accuracy when combined with other tests. This is illustrated in Tables $3-10$.

Although this argument is still completely heuristic it seems that the sporadic solutions for different pairs of $Y_{i}$ (or other tests) are independent enough that two pairs are sufficient to eliminate all potential "false" eigenvalues.

Given the striking "near-zero" singular values in the example above it is also tempting to try to use the singular values to detect Laplace eigenvalues. Unfortunately this is extremely inefficient both because of the number of arithmetic operations needed to compute singular values and also because these appear to be very sensitive to changes in the parameters. For instance, with $R$ as in the example and $R^{\prime}=R+1 \cdot 10^{-4}$ we obtain the singular values $s_{1}^{\prime}=0, s_{2}^{\prime}=1.7 \cdot 10^{-5}$ and $s_{3}^{\prime}=1.8 \cdot 10^{-5}$. It is in other words necessary to use a finer resolution than $10^{-4}$ to distinguish potential eigenvalues. Incidentally we have also found that there will be a number of sporadic values of $R$ for which the first non-zero singular value will be very small compared to the next larger.

\subsection{Pullback}

The existence of an efficient pullback algorithm which, given a $z \in \mathbb{H}$ finds a map $g \in G$ with $g z \in \mathcal{F}_{G}$ is essential. It is easy to see that a reduction using the generators $S: z \mapsto-1 / z$ and $T: z \mapsto z+1$ of $\Gamma$ where, in each step, $S, T$ or $T^{-1}$ is applied depending on whether $|z|<1, \Re z<-\frac{1}{2}$ or $>\frac{1}{2}$, produces a map $\gamma \in \Gamma$ with $\gamma z \in \mathcal{F}_{\Gamma}$ in 
a finite number of steps. It is then simply a matter of identifying the coset in $G \backslash \Gamma$ such that $\gamma^{-1} \in G V_{j}$. In this case $g=\gamma^{-1} V_{j}^{-1} \in G$ and $g z \in V_{j}\left(\mathcal{F}_{\Gamma}\right) \subseteq \mathcal{F}_{G}$.

For the system (20) to correspond to Maass cusp forms for the correct group it is important that the collection of pullbacks involve all generators of the group. It is easily seen that this will be the case if we involve all cosets, a condition which is much easier to verify.

\subsection{Eisenstein series and Scattering matrix}

It is not hard to modify the above method for computing Maass cusp forms to instead compute the Eisenstein series $E_{p}(z ; s)$ for a cusp $p$ and fixed $s$ and we will briefly indicate the necessary modifications. More details can be found in e.g. [5]. It is clear from the Fourier expansions (2) and (3) that we need to introduce constant terms

$$
c_{p i}(0 ; y ; s)=\delta_{p i} y^{s}+\varphi_{p i}(s) y^{1-s}
$$

in all steps. Treating $\varphi_{i j}(s)$ as a variable and $y^{s}$ as a constant and repeating the above leads to an inhomogeneous equation of the form

$$
V C=W+\llbracket 2 \varepsilon \rrbracket,
$$

where $C=\left(C_{j n}\right)$ is the vector of coefficients, $C_{j n}=c_{p j}(n ; s)$ if $n \neq 0$ and $C_{j 0}=\varphi_{p j}(s)$. The matrix coefficient are given by

$$
V_{n l}^{j i}=\frac{1}{2 Q} \sum_{I(m, j)=i} \kappa_{l}\left(y_{m j}^{*} ; s-1 / 2\right) e\left(l x_{m j}^{*}-n x_{m}\right)-\delta_{n l} \delta_{i j} \kappa_{n}(Y ; s-1 / 2)
$$

where $\kappa_{n}(y ; s-1 / 2)=\sqrt{y} K_{s-1 / 2}(2 \pi|n| y)$ if $n \neq 0$ and $\kappa_{0}(y ; s-1 / 2)=y^{1-s}$. The coefficients of the right hand side are then

$$
W_{l}^{j i}=\delta_{p j} \frac{1}{2 Q} \sum_{m=1-Q}^{Q}\left(y_{m}^{*}\right)^{s} e^{-2 \pi i n x_{m}}
$$

and since this is in general non-zero we find that (8.7) can be solved immediately without having to introduce any normalizations. It is important to once again remark that in general neither the constant term nor the other coefficients are known explicitly. Although the rapid decay of the $K$-Bessel function turns out again to sufficient to show that there exists a suitable truncation point $M_{0}=M\left(Y_{0}\right)$ for any fixed $s=\sigma+i t$ the situation is more delicate here since the coefficients can grow exponentially. From [21, Section 6.12] we know that if $1 / 2 \leq \sigma \leq 3 / 2$ and $s$ is bounded away from the residual spectrum then $\varphi_{p i}(s)=O(1)$ and $c_{p i}(n ; \sigma+i t)=O_{t}\left(e^{3|t|+Y_{0} \pi|n| / 4}\right)$ where $O_{t}$ indicates a constant depending on both $t$ and the group. More precisely, this constant depends on the zeros and poles of $\varphi(s)$.

It is clear that by setting up the system (8.7) simultanously for all Eisenstein series it is now possible to compute the scattering matrix $\Phi(s)$ as the matrix of all $\varphi_{j i}(s)$ and thus, in particular to find its poles and zeros numerically. 


\section{Completion of data}

In addition to locating and certifying potential eigenvalues it is also important to determine whether a list of such computed eigenvalues is complete or not. In Section 4 we briefly described how this could potentially be achieved rigorously using Turing's method, as done by Booker and Platt [10] for the modular group. Unfortunately, in the general subgroup case there are several obstacles to this and we have to settle for a heuristic version. With notation as in Theorem 4 let $N_{\exp }(T)$ be the numerically computed total discrete counting function $N(T)+N_{\text {res }}+N_{\text {exc }}, M_{\text {exp }}(T)$ a computed approximation of $M(T)$ and set

$$
E(T)=N_{\exp }(T)+M_{\exp }(T)-\frac{h}{12} T^{2}+\frac{e_{\infty}}{\pi} T \log (2 T / e)-c_{G},
$$

where

$$
c_{G}=-h \frac{1}{144}+e_{2} \frac{1}{8}+e_{3} \frac{2}{9}-e_{\infty} \frac{1}{4}+\frac{C}{2} \quad \text { and } \quad C=\frac{1}{2} \operatorname{Tr}(\mathbb{1}-\Phi(1 / 2)) .
$$

It is important to note that, in contrast to [29] and [10], we do not only need to compute the number of Maass cusp forms, but also the winding number and the number of exceptional and residual eigenvalues. Indeed, even the constant $c_{G}$, or more precisely, $\Phi(1 / 2)$, needs to be computed numerically. It follows directly from the functional equation (cf. e.g $\left[21\right.$, p. 296]) that $\Phi(1 / 2)^{2}=\mathbb{1}$ and hence $\Phi(1 / 2)$ is a diagonal matrix with entries \pm 1 . In the case of $\Gamma_{0}(N)$ for square-free $N$ it can be shown that $\Phi(1 / 2)=-\mathbb{1}$. See e.g. [21, Prop. 4.8 (p. 536)]. By using the algorithm described in Section 8.7 we found numerically that $\Phi(1 / 2)=-\mathbb{1}$ (up to an error of magnitude $10^{-16}$ using double precision) for all subgroups in the database, and in particular for all subgroups of index less than or equal to 17 .

The function $E(T)$ can now be used as follows to verify (heuristically) the completeness of a given list of tentative eigenvalues. Assuming that the list is complete (including exceptional and residual eigenvalues) we expect $E(T)$ to behave like $S(T)+g(T)$, in other words it should oscillate around zero and the average should satisfy

$$
\langle E(T)\rangle=\frac{1}{T} \int_{0}^{T} E(t) d t=O\left((\ln T)^{-2}\right) \quad \text { as } \quad T \rightarrow \infty .
$$

In case there are eigenvalues missing from the list we instead expect an oscillation around the negative integer $N_{\exp }(T)-N(T)$. For instance, a single missing eigenvalue would result in an oscillation around -1 .

Unfortunately this argument is not as easy to apply here as in e.g. [29] since it is impossible to distinguish between a missing discrete eigenvalue and a missing complete "turn" of the winding number. This phenomenon will be illustrated with an example in Section 10.4.

To compute $M_{\exp }(T)$ numerically we express $M(T)$ as $\frac{1}{2 \pi} \Delta_{0}^{T} \mathbf{A r g} \varphi(1 / 2+i t)$, in other words, as the change in argument of the scattering determinant $\varphi(1 / 2+i t)$ as $t$ ranges between 0 and $T$. It is then straight-forward to evaluate $\varphi(1 / 2+i t)$ over a set of grid points $t_{0}<t_{1}<\cdots<t_{k}$ and add up the changes in argument. The main difficulty here is that the argument changes very rapidly in the presence of zeros and poles close to the 
half-line (see Table 8). It is therefore necessary to adopt an adaptive approach where the step size is made smaller and smaller until the averages of $E(T)$ agree with predictions. If this does not occur then it might also be necessary to revisit the eigenvalue computation.

\subsection{Implementation}

All of the algorithms for working with Maass forms and subgroups of the modular group are implemented in Python with critical parts written in Cython and tied together with an interface based in Sage [59]. Some of the parts which were originally implemented by the author (in Fortran or Cython) are now implemented in GAP [18] and available through the Sage interface. For instance, the calculation of supergroups through block systems is now available Sage for subgroups of the modular group using GAP.

All source code used for computations in this paper is freely available in the GitHub repository [60] which contains a large number of algorithms by the author and others, for computing with different types of automorphic forms. A collection of examples and data relevant to the current paper can be found in [67].

\section{Numerical Results and Conclusions}

To conclude this paper we will give examples of computed data which illustrates key points from the previous sections.

\subsection{Subgroups}

Table 2 contains lists of specifications of conjugacy classes of subgroups of $\mathrm{PSL}_{2}(\mathbb{Z})$ of index less than or equal to 11 . The choice of including indices only up to 11 is simply motivated by the fact that printed the tables soon becomes unwieldy. A table with a complete classification of conjugacy classes up to index 17 as well as a selection of classes with index 18 are available at [67], which also contain information about how to work directly with a database of subgroups.

The structure of the table is as follows: we first list the index $h$ and then the signature $\left(h ; g, e_{\infty}, e_{2}, e_{3}\right)$ followed by a fixed permutation $\sigma_{S}$ which corresponds to the element $S \in \mathrm{PSL}_{2}(\mathbb{Z})$ and has $e_{2}$ fixed points. Following this we give a list of rows corresponding to representatives of $\mathrm{PGL}_{2}(\mathbb{Z})$-conjugacy classes of subgroups with the given signature and the choice of conjugate given by the permutation $\sigma_{S}$. For each such subgroup $G$ we give the permutations $\sigma_{R}$ and $\sigma_{T}$. In addition we also provide the columns $N,|(G)|$ and $h_{G}, k_{G}, C, n_{\text {sup }}$ and $\left\langle\sigma_{S}, \sigma_{R}\right\rangle$. Here $N$ is the generalized level, $|(G)|$ is the number of elements in the $\mathrm{PSL}_{2}(\mathbb{Z})$ conjugacy class of $G$ and $h_{G}=1$ if $G^{*}=J G J$ belongs to $(G)$, otherwise $h_{G}=2$. If $G^{*}=T^{-k} G T^{k}$ then $k_{G}=k$ if $J T^{-k}$ preserves the cusp classes, $k_{G}=k^{*}$ if it does not, and otherwise the field is left empty. In the $C$-column we write " $C$ " if the conjugacy class corresponds to a congruence subgroup and $n_{\text {sup }}$ is the number of supergroups. The final column, labelled $\left\langle\sigma_{S}, \sigma_{R}\right\rangle$ contains the common name, if it exist for the subgroup of $\mathrm{S}_{h}$ generated by $\sigma_{S}$ and $\sigma_{R}$. Note that this group is also isomorphic to the quotient $\mathrm{PSL}_{2}(\mathbb{Z}) / G^{N}$, where $G^{N}$ is the maximal normal subgroup of $G$. Most of these quotients are given by groups in standard notation, $S_{n}, A_{n}, \mathrm{PSL}_{p}(q), \mathrm{GL}_{p}(q)$ etc. The groups without a simple description, or if that description is too long, are described by their identifier in the GAP small group database [18]. The group Gap ${ }_{m, n}$ has order $m$ and the index in the database is $n$. It can be retrieved from the command line in 
GAP as "SmallGroup (m,n)". In some of the cases where a simple structural definition is available we provide this as well.

The existence of a reflectional symmetry $\alpha$ in the sense of Proposition 23 is indicated in the table by the fact that the $\mathrm{PSL}_{2}(\mathbb{Z})$ and $\mathrm{PGL}_{2}(\mathbb{Z})$ conjugacy classes coincide, in other words that $h_{G}=1$ and that $k_{G}$ is not empty. Note that if $h_{G}=1$ but $k_{G}$ is empty then the map which conjugates $G$ into $G^{*}$ does not preserve the cusp classes.

As an independent verification that we have at least been able to obtain the correct number of classes and subgroups we compare with the total number, $n_{h}$, of subgroups of a given index $h$, which can be computed using the recursive formula

$n_{h}=4 n_{h-3}+2 n_{h-4}+(h-3) n_{h-6}-2 n_{h-7}-(h-6) n_{h-9}+\sum_{i=1}^{h-7} n_{h-6-i} n_{i}-\sum_{i=1}^{h-10} n_{h-9-i} n_{i}$

given in [20] together with the initial values $n_{i}=1,1,4,8,5,22,42,40,120(1 \leq i \leq 9)$. This gives $n_{i}=265,286,764, \ldots$ for $i=10,11,12, \ldots$ and these numbers indeed agree with the count from the table up to index 17 . When referring to a particular group in the tables we use the notation $\Gamma\left(h ; g, \kappa, e_{2}, e_{3} ; N, i\right)$ to denote the $i$ th subgroup of signature $\left(h ; g, \kappa, e_{2}, e_{3}\right)$ and level $N$ in order of appearance in Table 2 . In case there is only one matching group the index $i$ is omitted. If we want to refer to a subgroup not in the tables then we give the signature, together with the permutation $\sigma_{T}$ where it is assumed that the corresponding $\sigma_{S}$ is normalized to have the form $(1)(2) \cdots\left(e_{2}\right)\left(e_{2}+1 e_{2}+2\right) \cdots(h-1 h)$.

To illustrate how permutations correspond to subgroups we include figures of fundamental domains for groups of index 7. In Figure 1(a) we see a fundamental domain for the group $\Gamma(7 ; 0,2,1,1 ; 6)$ and from Table 2 we see that the corresponding permutation

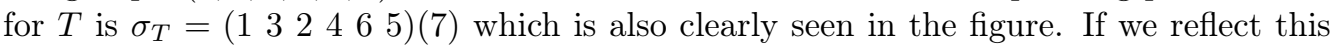
group then we obtain a group with $\sigma_{T}^{*}=\left(\begin{array}{llllll}1 & 5 & 6 & 4 & 2 & 3\end{array}\right)(7)$ which is also visible in Figure 1 (b). Observe that the standard fundamental domain for $\mathrm{PSL}_{2}(\mathbb{Z})$ is the copy labelled with 1 and this is the copy with center along the imaginary axis. Similar correspondences between fundamental domains and permutations can be seen in Figures 1(c) and 1(d).

\begin{tabular}{|c|c|c|c|c|c|c|c|c|c|}
\hline$\mu$ & $\begin{array}{l}\left(\mu ; g, \kappa, e_{2}, e_{3}\right) \\
\sigma_{R}\end{array}$ & $\begin{array}{l}\sigma_{S} \\
\sigma_{T} \\
\end{array}$ & $N$ & $|(G)|$ & $h_{G}$ & $k_{G}$ & $C$ & $n_{\text {sup }}$ & $\left\langle\sigma_{S}, \sigma_{R}\right\rangle$ \\
\hline 2 & $\begin{array}{l}(2 ; 0,1,0,2) \\
(1)(2)\end{array}$ & $\begin{array}{l}\left(\begin{array}{ll}1 & 2\end{array}\right) \\
\left(\begin{array}{ll}1 & 2\end{array}\right)\end{array}$ & 2 & 1 & 1 & 0 & $C$ & 0 & $S_{2}$ \\
\hline 3 & $\begin{array}{l}(3 ; 0,1,3,0) \\
(123)\end{array}$ & $\begin{array}{l}(1)(2)(3) \\
\left(\begin{array}{lll}1 & 2 & 3\end{array}\right)\end{array}$ & 3 & 1 & 1 & 0 & $C$ & 0 & $A_{3}$ \\
\hline & 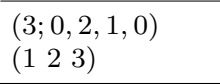 & $\begin{array}{l}(1)\left(\begin{array}{ll}2 & 3\end{array}\right) \\
(12)(3)\end{array}$ & 2 & 3 & 1 & 0 & $C$ & 0 & $S_{3}$ \\
\hline 4 & $\begin{array}{l}(4 ; 0,1,2,1) \\
(124)(3)\end{array}$ & $\begin{array}{l}\left(\begin{array}{l}1 \\
1\end{array}\right)\left(\begin{array}{lll}2 & (3 & 4\end{array}\right) \\
\left(\begin{array}{llll}1 & 2 & 4 & 3\end{array}\right)\end{array}$ & 4 & 4 & 1 & 1 & $C$ & 0 & $S_{4}$ \\
\hline & $\begin{array}{l}(4 ; 0,2,0,1) \\
(1)(234)\end{array}$ & $\begin{array}{l}\left(\begin{array}{lll}1 & 2\end{array}\right)\left(\begin{array}{ll}3 & 4\end{array}\right) \\
\left(\begin{array}{lll}1 & 3 & 2\end{array}\right)\left(\begin{array}{l}4\end{array}\right)\end{array}$ & 3 & 4 & 1 & 2 & $C$ & 0 & $A_{4}$ \\
\hline 5 & $\begin{array}{l}(5 ; 0,1,1,2) \\
(135)(2)(4) \\
\end{array}$ & 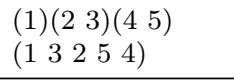 & 5 & 5 & 1 & 0 & $C$ & 0 & $A_{5}$ \\
\hline 6 & $\begin{array}{l}(6 ; 0,1,0,3) \\
(1)(246)(3)(5)\end{array}$ & 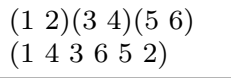 & 6 & 2 & 1 & 1 & $C$ & 1 & $C_{3} \times S_{3}$ \\
\hline & $(6 ; 0,1,4,0)$ & $\begin{array}{r}(1)(2)(3)(4)(56) \\
3\end{array}$ & & & & & & & \\
\hline
\end{tabular}




\begin{tabular}{|c|c|c|c|c|c|c|c|c|c|}
\hline & $\left(\begin{array}{lll}1 & 2 & 5\end{array}\right)(346)$ & $(1253346)$ & 6 & 3 & 1 & 1 & $C$ & 1 & $C_{2} \times A_{4}$ \\
\hline & $\begin{array}{l}(6 ; 1,1,0,0) \\
(135)(246)\end{array}$ & 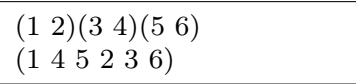 & 6 & 1 & 1 & 0 & $C$ & 2 & $C_{6}$ \\
\hline & 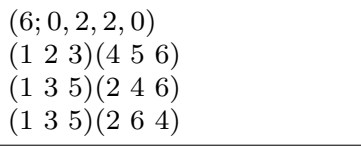 & 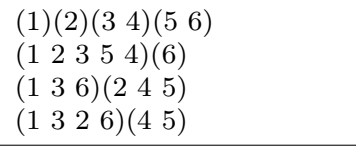 & $\begin{array}{l}5 \\
3 \\
4 \\
\end{array}$ & $\begin{array}{l}6 \\
3 \\
3 \\
\end{array}$ & $\begin{array}{l}1 \\
1 \\
1 \\
\end{array}$ & $\begin{array}{l}1 \\
0 \\
0 \\
\end{array}$ & $\begin{array}{l}C \\
C \\
C \\
\end{array}$ & $\begin{array}{l}0 \\
1 \\
1 \\
\end{array}$ & $\begin{array}{l}A_{5} \\
A_{4} \\
S_{4} \\
\end{array}$ \\
\hline & 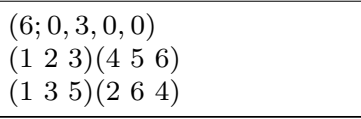 & 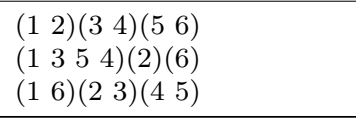 & $\begin{array}{l}4 \\
2 \\
\end{array}$ & $\begin{array}{l}3 \\
1 \\
\end{array}$ & $\begin{array}{l}1 \\
1\end{array}$ & $\begin{array}{l}0 \\
0\end{array}$ & $\begin{array}{l}C \\
C \\
\end{array}$ & $\begin{array}{l}1 \\
4\end{array}$ & $\begin{array}{l}S_{4} \\
S_{3} \\
\end{array}$ \\
\hline 7 & $\begin{array}{l}(7 ; 0,1,3,1) \\
(126)(357)(4)\end{array}$ & $\begin{array}{l}(1)(2)(3)(45)(67) \\
(1263547)\end{array}$ & 7 & 7 & 2 & & $C$ & 0 & $\mathrm{PSL}_{2}(7)$ \\
\hline & 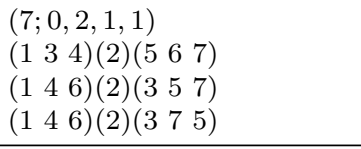 & $\begin{array}{l}\left(\begin{array}{lllll}1 & (2 & 3\end{array}\right)\left(\begin{array}{llll}4 & 5\end{array}\right)\left(\begin{array}{ll}6 & 7\end{array}\right) \\
\left(\begin{array}{lllllll}1 & 3 & 2 & 4 & 6 & 5\end{array}\right)(7) \\
\left(\begin{array}{lllll}1 & 4 & 7\end{array}\right)\left(\begin{array}{llll}2 & 5 & 6 & 3\end{array}\right) \\
\left(\begin{array}{llllll}1 & 4 & 3 & 2 & 7\end{array}\right)\left(\begin{array}{lll}5 & 6\end{array}\right) \\
\end{array}$ & $\begin{array}{c}6 \\
12 \\
10 \\
\end{array}$ & $\begin{array}{l}7 \\
7 \\
7 \\
\end{array}$ & $\begin{array}{l}2 \\
1 \\
1 \\
\end{array}$ & $\begin{array}{l}0 \\
0 \\
\end{array}$ & & $\begin{array}{l}0 \\
0 \\
0 \\
\end{array}$ & $\begin{array}{l}\mathrm{Gap}_{42,1} \\
S_{7} \\
S_{7} \\
\end{array}$ \\
\hline 8 & 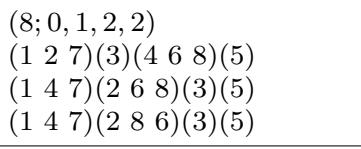 & 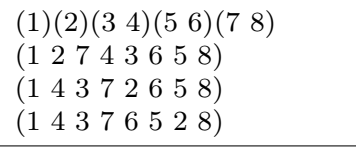 & $\begin{array}{l}8 \\
8 \\
8 \\
\end{array}$ & $\begin{array}{l}8 \\
4 \\
8 \\
\end{array}$ & $\begin{array}{l}1 \\
2 \\
1 \\
\end{array}$ & $\begin{array}{l}1 \\
6\end{array}$ & $C$ & $\begin{array}{l}0 \\
1 \\
0\end{array}$ & $\begin{array}{l}\operatorname{PSL}_{3}(2) \ltimes C_{2} \\
\mathrm{GL}_{2}(3) \\
\operatorname{PSL}_{3}(2) \ltimes C_{2} \\
\end{array}$ \\
\hline & 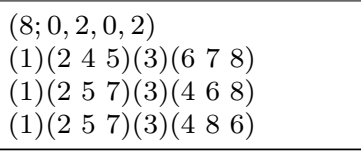 & 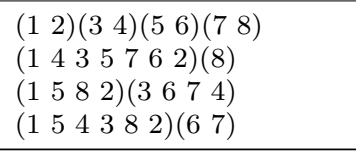 & $\begin{array}{l}7 \\
4 \\
6 \\
\end{array}$ & $\begin{array}{l}8 \\
4 \\
4 \\
\end{array}$ & $\begin{array}{l}1 \\
1 \\
1 \\
\end{array}$ & $\begin{array}{l}1 \\
3 \\
2 \\
\end{array}$ & $\begin{array}{l}C \\
C \\
C \\
\end{array}$ & $\begin{array}{l}0 \\
2 \\
2 \\
\end{array}$ & $\begin{array}{l}\mathrm{PSL}_{2}(7) \\
S_{4} \\
C_{2} \times A_{4} \\
\end{array}$ \\
\hline 9 & $\begin{array}{l}(9 ; 1,1,1,0) \\
(124)(368)(579)\end{array}$ & 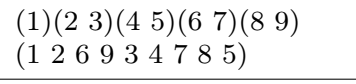 & 9 & 9 & 1 & 0 & & 0 & $\mathrm{PSL}_{2}(8)$ \\
\hline & $\begin{array}{l}(9 ; 0,1,5,0) \\
(126)(348)(579)\end{array}$ & $\begin{array}{l}(1)(2)(3)(4)(5)(67)(89) \\
(126934857)\end{array}$ & 9 & 9 & 1 & 5 & $C$ & 1 & $\mathrm{Gap}_{324,160}$ \\
\hline & $\begin{array}{l}(9 ; 0,1,1,3) \\
(138)(2)(4)(579)(6)\end{array}$ & 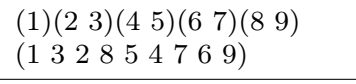 & 9 & 9 & 2 & & & 0 & $\mathrm{PSL}_{2}(8) \ltimes C_{3}$ \\
\hline & 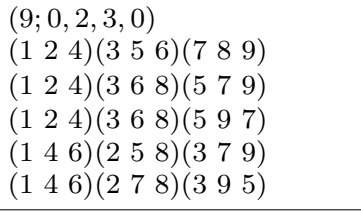 & 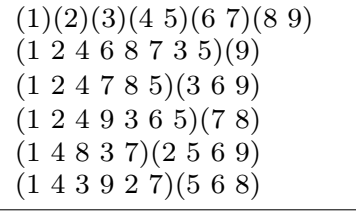 & $\begin{array}{c}8 \\
6 \\
14 \\
20 \\
6 \\
\end{array}$ & $\begin{array}{l}9 \\
9 \\
9 \\
9 \\
3 \\
\end{array}$ & $\begin{array}{l}2 \\
1 \\
1 \\
1 \\
1\end{array}$ & $\begin{array}{l}1 \\
1 \\
3 \\
0\end{array}$ & $C$ & $\begin{array}{l}0 \\
1 \\
0 \\
0 \\
2\end{array}$ & $\begin{array}{l}\operatorname{Gap}_{432,734} \\
\operatorname{Gap}_{54,5} \\
S_{9} \\
S_{9} \\
C_{3} \times S_{3} \\
\end{array}$ \\
\hline & 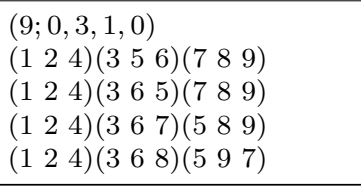 & 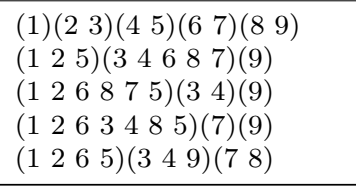 & $\begin{array}{c}15 \\
6 \\
7 \\
12 \\
\end{array}$ & $\begin{array}{l}9 \\
9 \\
9 \\
9 \\
\end{array}$ & $\begin{array}{c}1 \\
1 \\
1^{*} \\
1\end{array}$ & $\begin{array}{l}0 \\
0 \\
0 \\
0 \\
\end{array}$ & & $\begin{array}{l}0 \\
1 \\
0 \\
1 \\
\end{array}$ & $\begin{array}{l}A_{9} \\
\operatorname{Gap}_{54,5} \\
\operatorname{PSL}_{2}(8) \\
\operatorname{Gap}_{648,703} \\
\end{array}$ \\
\hline 10 & 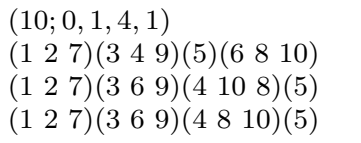 & 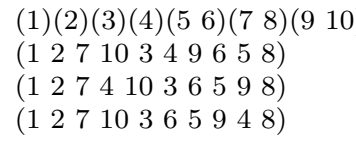 & $\begin{array}{l}0) \\
10 \\
10 \\
10\end{array}$ & $\begin{array}{l}10 \\
10 \\
10\end{array}$ & $\begin{array}{l}1 \\
2 \\
2\end{array}$ & 5 & & $\begin{array}{l}0 \\
0 \\
0\end{array}$ & $\begin{array}{l}S_{10} \\
S_{10} \\
S_{10}\end{array}$ \\
\hline & $\begin{array}{l}(10 ; 0,1,0,4) \\
(1)(249)(3)(5)(6 \quad 810)(7)\end{array}$ & 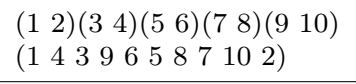 & 10 & 5 & 1 & 1 & $C$ & 2 & $C_{2} \times A_{5}$ \\
\hline & $\begin{array}{l}(10 ; 1,1,0,1) \\
(1)(235)(479)(6810)\end{array}$ & 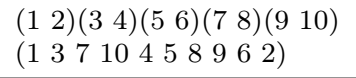 & 10 & 10 & 1 & 9 & & 0 & $A_{6} \ltimes C_{2}$ \\
\hline & $(10 ; 0,2,2,1)$ & $(1)(2)(34)(5 \quad 6)(78)(9$ 10) & & & & & & & \\
\hline
\end{tabular}




\begin{tabular}{|c|c|c|c|c|c|c|c|c|c|}
\hline & 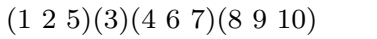 & $(125798436)(10)$ & 9 & 10 & 2 & & & 0 & $A_{10}$ \\
\hline & $\left(\begin{array}{lll}1 & 2 & 5\end{array}\right)(3)\left(\begin{array}{lll}4 & 7 & 9\end{array}\right)\left(\begin{array}{lll}6 & 10 & 8\end{array}\right)$ & 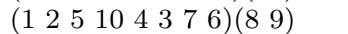 & 8 & 10 & 1 & 1 & & 0 & $A_{10}$ \\
\hline & $\left(\begin{array}{lll}1 & 2 & 5\end{array}\right)(3)\left(4 \begin{array}{llll}4 & 9\end{array}\right)\left(\begin{array}{lll}6 & 8 & 10\end{array}\right)$ & $\left(\begin{array}{lllll}1 & 2 & 5 & 8 & 9\end{array}\right)\left(\begin{array}{llll}3 & 7 & 10 & 4\end{array}\right)$ & 12 & 10 & 1 & 1 & & 0 & $A_{10}$ \\
\hline & $(145)(267)(3)(8910)$ & $(143579826)(10)$ & 9 & 10 & 2 & & & 0 & $A_{10}$ \\
\hline & $(145)(279)(3)(6108)$ & 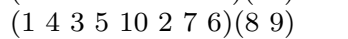 & 8 & 10 & 2 & & & 0 & $A_{10}$ \\
\hline & $(145)(276)(3)(8910)$ & $(143527986)(10)$ & 9 & 10 & 2 & & & 0 & $A_{10}$ \\
\hline & $(145)(279)(3)(6 \quad 810)$ & $(1435896)(2710)$ & 21 & 10 & 2 & & & 0 & $A_{10}$ \\
\hline & $(155)(2 \quad 69)(3)(4810)$ & 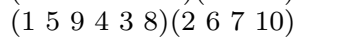 & 12 & 10 & 2 & & & 0 & $A_{10}$ \\
\hline & $(157)(289)(3)(4106)$ & 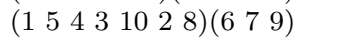 & 21 & 10 & 1 & 5 & & 0 & $A_{10}$ \\
\hline & $(157)(289)(3)(4610)$ & $\left(\begin{array}{lllll}1 & 5 & 10 & 2 & 8\end{array}\right)\left(\begin{array}{lllll}3 & 6 & 7 & 9 & 4\end{array}\right)$ & 5 & 10 & 1 & 3 & $C$ & 0 & $A_{5}$ \\
\hline & $(10 ; 0,3,0,1)$ & $(12)(34)(56)(78)(910)$ & & & & & & & \\
\hline & $(1)(235)(467)(8910)$ & $(1362)(45798)(10)$ & 20 & 10 & 1 & 3 & & 0 & $S_{10}$ \\
\hline & $(1)(235)(479)\left(\begin{array}{lll}6 & 10 & 8\end{array}\right)$ & 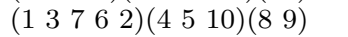 & 30 & 10 & 1 & 4 & & 0 & $S_{10}$ \\
\hline & $(1)(235)(476)(8910)$ & $(13798662)(45)(10)$ & 14 & 10 & 1 & 6 & & 0 & $S_{10}$ \\
\hline & 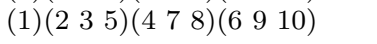 & $(137445962)(8)(10)$ & 8 & 10 & 1 & $7^{*}$ & & 0 & $A_{6} \ltimes C_{2}$ \\
\hline 11 & $(11 ; 0,1,3,2)$ & $(1)(2)(3)(45)(67)(89)(10$ & $11)$ & & & & & & \\
\hline & $(128)(3105)(4)(6)(7119)$ & $(1287611543109)$ & 11 & 11 & 2 & & $C$ & 0 & $\mathrm{PSL}_{2}(11)$ \\
\hline & $\left(\begin{array}{lll}1 & 2 & 8\end{array}\right)\left(\begin{array}{lll}3 & 10 & 5\end{array}\right)(4)(6)\left(7 \begin{array}{ll}7 & 9\end{array}\right)$ & $\left(\begin{array}{lllllllllll}1 & 2 & 8 & 11 & 5 & 4 & 3 & 10 & 7 & 6 & 9\end{array}\right)$ & 11 & 11 & 2 & & & 0 & $A_{11}$ \\
\hline & $\left(\begin{array}{lll}1 & 2 & 8\end{array}\right)(3 \quad 109)(4)(5 \quad 7 \quad 11)(6)$ & $\left(\begin{array}{llllllllll}1 & 2 & 8 & 3 & 10 & 5 & 4 & 7 & 6 & 11\end{array}\right)$ & 11 & 11 & 2 & & & 0 & $A_{11}$ \\
\hline & $(158)(2107)(3119)(4)(6)$ & $\left(\begin{array}{llllllllll}1 & 5 & 4 & 8 & 3 & 11 & 7 & 6 & 2 & 10\end{array}\right)$ & 11 & 11 & 1 & 8 & & 0 & $A_{11}$ \\
\hline & $(1558)(2710)(3911)(4)(6)$ & $\left(\begin{array}{llllllllll}1 & 5 & 4 & 8 & 11 & 2 & 7 & 6 & 10 & 3\end{array}\right)$ & 11 & 11 & 2 & & & 0 & $A_{11}$ \\
\hline & $(158)(2910)(3117)(4)(6)$ & $(1548107631129)$ & 11 & 11 & 1 & 7 & & 0 & $A_{11}$ \\
\hline & $(11 ; 0,2,1,2)$ & $(1)(23)(45)(67)(89)(10$ & 11) & & & & & & \\
\hline & 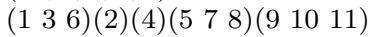 & $(13268109547)(11)$ & 10 & 11 & 2 & & & 0 & $S_{11}$ \\
\hline & $\left(\begin{array}{lll}1 & 3 & 6\end{array}\right)(2)(4)\left(\begin{array}{lll}5 & 8 & 10\end{array}\right)\left(\begin{array}{lll}7 & 11 & 9\end{array}\right)$ & $\left(\begin{array}{lllllllll}1 & 3 & 2 & 6 & 11 & 5 & 4 & 8 & 7\end{array}\right)\left(\begin{array}{ll}9 & 10\end{array}\right)$ & 18 & 11 & 2 & & & 0 & $S_{11}$ \\
\hline & 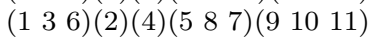 & $(13265481097)(11)$ & 10 & 11 & 2 & & & 0 & $S_{11}$ \\
\hline & $\left(\begin{array}{lll}1 & 3 & 6\end{array}\right)(2)(4)\left(\begin{array}{lll}5 & 8 & 10\end{array}\right)\left(\begin{array}{lll}7 & 9 & 11\end{array}\right)$ & $\left(\begin{array}{lllllll}1 & 3 & 2 & 6 & 9 & 10 & 7\end{array}\right)\left(\begin{array}{llll}4 & 8 & 11 & 5\end{array}\right)$ & 28 & 11 & 2 & & & 0 & $S_{11}$ \\
\hline & $\left(\begin{array}{lll}1 & 6 & 8\end{array}\right)(2)\left(\begin{array}{lll}3 & 10 & 7\end{array}\right)(4)\left(\begin{array}{lll}5 & 11 & 9\end{array}\right)$ & 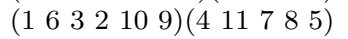 & 30 & 11 & 2 & & & 0 & $S_{11}$ \\
\hline & $\left(\begin{array}{lll}1 & 6 & 8\end{array}\right)(2)\left(\begin{array}{lll}3 & 5 & 10\end{array}\right)(4)\left(7 \begin{array}{ll}7 & 11\end{array}\right)$ & 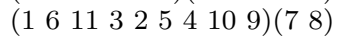 & 18 & 11 & 1 & 0 & & 0 & $S_{11}$ \\
\hline & $\left(\begin{array}{lll}1 & 6 & 8\end{array}\right)(2)\left(\begin{array}{lll}3 & 5 & 10\end{array}\right)\left(\begin{array}{l}4 \\
4\end{array}\right)\left(\begin{array}{lll}7 & 9 & 11\end{array}\right)$ & $\left(\begin{array}{lll}1 & 6 & 9\end{array}\right)\left(\begin{array}{lllllll}2 & 5 & 4 & 10 & 7 & 8 & 11\end{array}\right)$ & 24 & 11 & 1 & 0 & & 0 & $S_{11}$ \\
\hline & $\left(\begin{array}{lll}1 & 6 & 8\end{array}\right)(2)\left(\begin{array}{lll}3 & 5 & 7\end{array}\right)(4)\left(\begin{array}{lll}9 & 10 & 11\end{array}\right)$ & $\left(\begin{array}{llllllllll}1 & 6 & 3 & 2 & 5 & 4 & 7 & 8 & 10 & 9\end{array}\right)(11)$ & 10 & 11 & 2 & & & 0 & $S_{11}$ \\
\hline & $\left(\begin{array}{lll}1 & 6 & 8\end{array}\right)(2)\left(\begin{array}{lll}3 & 7 & 10\end{array}\right)(4)\left(\begin{array}{llll}5 & 11 & 9\end{array}\right)$ & 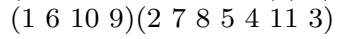 & 28 & 11 & 1 & 0 & & 0 & $S_{11}$ \\
\hline & $\left(\begin{array}{lll}1 & 6 & 8\end{array}\right)(2)\left(\begin{array}{lll}3 & 9 & 10\end{array}\right)(4)\left(\begin{array}{lll}5 & 11 & 7\end{array}\right)$ & 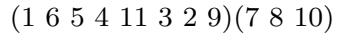 & 24 & 11 & 1 & 0 & & 0 & $S_{11}$ \\
\hline
\end{tabular}

Table 2: Conjugacy classes of subgroups of $\operatorname{PSL}_{2}(\mathbb{Z})$ with index at most 11

\subsection{Maass forms}

The spectrum of all subgroups mentioned in the previous section, and in particular all subgroups of index up to and including 17 , has been numerically investigated. As expected we found newforms on all congruence subgroups and all cycloidal subgroups.

In addition, we also found newforms (in the sense of Section 3) on precisely those noncongruence, non-cycloidal subgroups which satisfy Theorem 26. In this case, all newforms we found were $\alpha$-odd and the number of newforms were approximately one half of what is indicated by Weyl's law for the surface $G \backslash \mathcal{H}$, as is to be expected for the $\alpha$-odd space $L_{-}^{2}(G \backslash \mathcal{H})$. (In this vein, cf. [57, pp. 448 (footnote 1), 670 (middle)].) Since our investigation has been directed at investigating as many groups as possible, we have generally not taken $R$ beyond 10 or so for most groups. Although 10 is not very large by any stretch, a view at the refined Weyl's law in Theorem 4 this corresponds to the error terms being $|g(T)|<0.15$ (for all groups in the database) and $\log (T)^{-2}<0.2$. Assuming the implied constant in the latter is, say 1 , we still have an average Weyl's law with an error smaller than 0.22. This will be discussed in more detail in Section 10.4. 


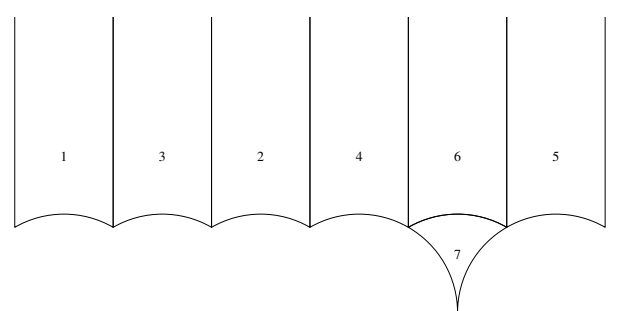

(a) $\Gamma(7 ; 0,2,1,1 ; 6)$

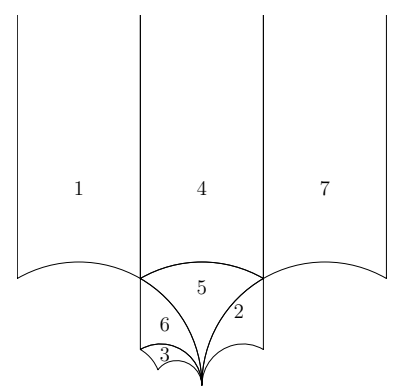

(c) $\Gamma(7 ; 0 ; 2,1,1 ; 12)$

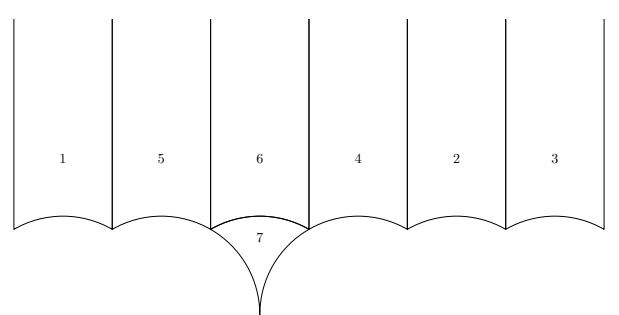

(b) $\Gamma(7 ; 0 ; 2,1,1 ; 6)^{*}$

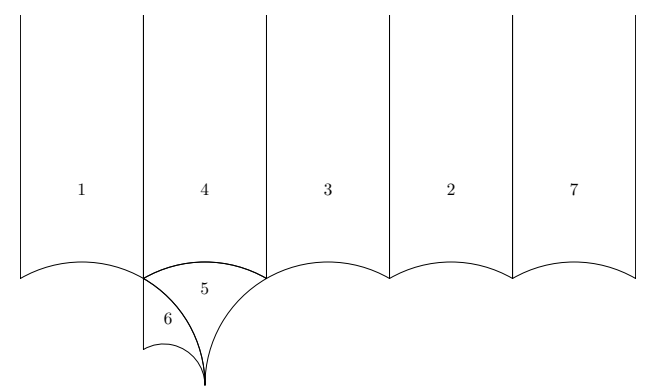

(d) $\Gamma(7 ; 0 ; 2,1,1 ; 10)$

Figure 1: Fundamental domains for groups of index 7

In the case of noncongruence, non-cycloidal groups which do not satisfy Theorem 23, we found no newforms whatsoever. An example of a group satisfying $\left(G^{*}\right)=(G)$, but that violates the cusp class preserving condition is $\Gamma(10 ; 0,3,0,1 ; 8)$. These observations are the foundations for our believe in Conjecture 2.

When testing for existence/nonexistence of newforms we ran the program up to height $R=10$ for indexes $\leq 10$ and up to $R=5$ for larger indices. In all cases of non-congruence, non-cycloidal groups that we have checked, all newforms found have been of $\alpha$-odd type. For the cycloidal groups, we found both $\alpha$-even, $\alpha$-odd and in certain cases nonsymmetric newforms, as expected from formula. For a flavor of what the spectrum of a noncongruence subgroup look like see e.g. Tables 3-6. See also Figure 2 for density plots , i.e. plots of $|\varphi|^{2}$, with Maass forms for the group $\Gamma(7 ; 0,2,1,1 ; 10)$. Red corresponds to larger values and dark blue to smaller values. 


\begin{tabular}{cc}
\hline \multicolumn{2}{c}{$\Gamma(7 ; 0,2,1,1 ; 10 ; 1)$} \\
\cline { 2 - 2 } & $|c(-1)+1|$ \\
\hline 2.98380890177954 & $7 \cdot 10^{-12}$ \\
4.25864509747714 & $1 \cdot 10^{-12}$ \\
4.89540660025545 & $2 \cdot 10^{-13}$ \\
5.28884863158981 & $1 \cdot 10^{-12}$ \\
6.15234838528998 & $1 \cdot 10^{-16}$ \\
6.27199072998424 & $2 \cdot 10^{-13}$ \\
6.76036517325739 & $2 \cdot 10^{-13}$ \\
7.20603858428836 & $4 \cdot 10^{-13}$ \\
7.55764967370570 & $4 \cdot 10^{-13}$ \\
7.77456459246736 & $6 \cdot 10^{-14}$ \\
8.04276906236577 & $4 \cdot 10^{-13}$ \\
8.50359685011489 & $8 \cdot 10^{-13}$ \\
8.77366479019267 & $3 \cdot 10^{-13}$ \\
8.88752983993304 & $2 \cdot 10^{-13}$ \\
9.29204073179663 & $5 \cdot 10^{-14}$ \\
$9.53369526135354 *$ & $2 \cdot 10^{-14}$ \\
9.67736151045037 & $4 \cdot 10^{-13}$ \\
\hline$*$ Oldform from $\mathrm{PSL}_{2}(\mathbb{Z})$.
\end{tabular}

Table 3: Eigenvalues $\lambda=1 / 4+R^{2}$ for a noncongruence subgroup of index 7

\begin{tabular}{cc}
\hline \multicolumn{2}{c}{$\Gamma(9 ; 0,2,3,0 ; 20)$} \\
\hline$R$ & $\left|c(-1)-e^{\frac{2 \pi i}{10}}\right|$ \\
\hline 2.05495976063979 & $6 \cdot 10^{-11}$ \\
3.25563226951552 & $1 \cdot 10^{-12}$ \\
4.01743421448455 & $8 \cdot 10^{-13}$ \\
4.39990456866076 & $3 \cdot 10^{-13}$ \\
4.92041181302557 & $1 \cdot 10^{-13}$ \\
5.22354223285616 & $1 \cdot 10^{-11}$ \\
5.70296517107618 & $2 \cdot 10^{-14}$ \\
6.20783400944797 & $3 \cdot 10^{-12}$ \\
6.52618304206566 & $8 \cdot 10^{-13}$ \\
6.78783029888573 & $4 \cdot 10^{-13}$ \\
7.07791461588707 & $2 \cdot 10^{-12}$ \\
7.38877048107201 & $5 \cdot 10^{-15}$ \\
8.02743455884534 & $3 \cdot 10^{-10}$ \\
8.70656893384487 & $2 \cdot 10^{-13}$ \\
8.81164911527479 & $6 \cdot 10^{-15}$ \\
9.03359858422541 & $9 \cdot 10^{-11}$ \\
9.50035839336283 & $2 \cdot 10^{-11}$ \\
$9.53369526135354 *$ & $5 \cdot 10^{-09}$ \\
9.66113248208174 & $3 \cdot 10^{-11}$ \\
\hline
\end{tabular}

\begin{tabular}{cc}
\hline \multicolumn{2}{c}{$\Gamma(9 ; 0,3,1,0 ; 15)$} \\
\hline$R$ & $|c(-1)+1|$ \\
\hline 3.11274526127958 & $8 \cdot 10^{-13}$ \\
3.79758314524666 & $8 \cdot 10^{-14}$ \\
4.68277129730610 & $2 \cdot 10^{-14}$ \\
5.61420134556306 & $9 \cdot 10^{-12}$ \\
6.17840605444398 & $4 \cdot 10^{-14}$ \\
6.28023134316972 & $2 \cdot 10^{-11}$ \\
6.50085225008714 & $8 \cdot 10^{-10}$ \\
6.87257413990397 & $8 \cdot 10^{-09}$ \\
7.21783938535166 & $2 \cdot 10^{-10}$ \\
7.41980107735753 & $2 \cdot 10^{-11}$ \\
7.73811611339896 & $2 \cdot 10^{-12}$ \\
7.89543101394190 & $3 \cdot 10^{-10}$ \\
8.33429557635011 & $5 \cdot 10^{-11}$ \\
8.82147607539715 & $4 \cdot 10^{-08}$ \\
8.83035851126317 & $6 \cdot 10^{-09}$ \\
9.17711548223084 & $3 \cdot 10^{-10}$ \\
9.23041542661088 & $6 \cdot 10^{-08}$ \\
9.53369526135299 & $4 \cdot 10^{-09}$ \\
9.73675250028969 & $3 \cdot 10^{-09}$ \\
\hline
\end{tabular}

* Oldform from $\mathrm{PSL}_{2}(\mathbb{Z})$.

Table 4: Eigenvalues $\lambda=1 / 4+R^{2}$ for noncongruence subgroups of index 9 


\begin{tabular}{|c|c|c|}
\hline \multicolumn{3}{|c|}{$\Gamma(10 ; 1,1,0,1 ; 10 ; 1)$} \\
\hline$R$ & $\left|c(-1)-e^{\frac{9 \pi i}{5}}\right|$ & $\left|c(-1)+e^{\frac{9 \pi i}{5}}\right|$ \\
\hline $1.56460530796110^{\mathrm{e}}$ & $6 \cdot 10^{-11}$ & \\
\hline $2.11939343023923^{\circ}$ & & $1 \cdot 10^{-11}$ \\
\hline $2.50414532509967^{\mathrm{e}}$ & $1 \cdot 10^{-09}$ & \\
\hline $2.91592762476298^{\mathrm{e}}$ & $6 \cdot 10^{-10}$ & \\
\hline $3.00023468927910^{\circ}$ & & $2 \cdot 10^{-11}$ \\
\hline $3.10845111145684^{\circ}$ & & $1 \cdot 10^{-13}$ \\
\hline $3.56237482722476^{\mathrm{e}}$ & $6 \cdot 10^{-11}$ & \\
\hline $3.65766022871834^{\circ}$ & & $1 \cdot 10^{-12}$ \\
\hline $3.88274850431287^{\circ}$ & & $2 \cdot 10^{-12}$ \\
\hline $4.10694094068138^{\mathrm{e}}$ & $6 \cdot 10^{-11}$ & \\
\hline $4.55947270613401^{\circ}$ & & $4 \cdot 10^{-12}$ \\
\hline $4.74782424736486^{\circ}$ & & $3 \cdot 10^{-13}$ \\
\hline $4.76752492536872^{\mathrm{e}}$ & $6 \cdot 10^{-11}$ & \\
\hline $4.84953817531720^{\mathrm{e}}$ & $1 \cdot 10^{-10}$ & \\
\hline
\end{tabular}

e and ${ }^{\circ}$ denotes even and odd with respect to $z \mapsto-\bar{z}+9$.

Table 5: Eigenvalues $\lambda=1 / 4+R^{2}$ for a cycloidal noncongruence subgroup.

\begin{tabular}{|c|c|c|c|}
\hline \multicolumn{2}{|c|}{$\Gamma(10 ; 0,2,2,1 ; 12)$} & \multicolumn{2}{|c|}{$\Gamma(10 ; 0,2,2,1 ; 8)$} \\
\hline$R$ & $\left|c(-1)+e^{\frac{2 \pi i}{6}}\right|$ & $R$ & $\left|c(-1)+e^{\frac{2 \pi i}{8}}\right|$ \\
\hline 2.7818731828528 & $5 \cdot 10^{-11}$ & 2.4093187748458 & $1 \cdot 10^{-10}$ \\
\hline 3.3718432084177 & $5 \cdot 10^{-11}$ & 3.2980390023681 & $7 \cdot 10^{-09}$ \\
\hline 3.7603510695428 & $7 \cdot 10^{-12}$ & 3.7095318601808 & $9 \cdot 10^{-12}$ \\
\hline 4.2298571733650 & $2 \cdot 10^{-12}$ & 4.2745965949785 & $3 \cdot 10^{-10}$ \\
\hline 4.8535236823625 & $5 \cdot 10^{-12}$ & 4.5864668030104 & $4 \cdot 10^{-10}$ \\
\hline \multicolumn{2}{|c|}{$\Gamma(10 ; 0,2,2,1 ; 21,2)$} & \multicolumn{2}{|c|}{$\Gamma(10 ; 0,3,0,1 ; 30,1)$} \\
\hline$R$ & $\left|c(-1)+e^{\frac{5 \pi i}{7}}\right|$ & $R$ & $\left|c(-1)+e^{\frac{4 \pi i}{5}}\right|$ \\
\hline 1.9099559058915 & $3 \cdot 10^{-08}$ & 2.6817198332904 & $8 \cdot 10^{-09}$ \\
\hline 3.2045604949759 & $6 \cdot 10^{-09}$ & 3.5645125659019 & $4 \cdot 10^{-11}$ \\
\hline 3.5047586985069 & $6 \cdot 10^{-09}$ & 4.4420512498009 & $1 \cdot 10^{-11}$ \\
\hline 4.2075358132024 & $2 \cdot 10^{-10}$ & 4.7018937088526 & $5 \cdot 10^{-12}$ \\
\hline 4.7774437178153 & $2 \cdot 10^{-08}$ & 4.9743710668935 & $3 \cdot 10^{-11}$ \\
\hline \multicolumn{2}{|c|}{$\Gamma(10 ; 0,3,0,1 ; 20,1)$} & \multicolumn{2}{|c|}{$\Gamma(10 ; 0,3,0,1 ; 14,1)$} \\
\hline$R$ & $\left|c(-1)+e^{\frac{3 \pi i}{4}}\right|$ & $R$ & $\left|c(-1)+e^{\frac{6 \pi i}{7}}\right|$ \\
\hline 3.0877339408933 & $2 \cdot 10^{-09}$ & 2.5744038863311 & $3 \cdot 10^{-11}$ \\
\hline 3.5070902176928 & $2 \cdot 10^{-11}$ & 3.7941339745816 & $2 \cdot 10^{-10}$ \\
\hline \multirow[t]{2}{*}{4.3456594421154} & $5 \cdot 10^{-12}$ & 4.0617322627988 & $8 \cdot 10^{-11}$ \\
\hline & & 4.6861370442514 & $7 \cdot 10^{-11}$ \\
\hline
\end{tabular}

Table 6: Eigenvalues $\lambda=1 / 4+R^{2}$ for noncongruence subgroups of index 10 


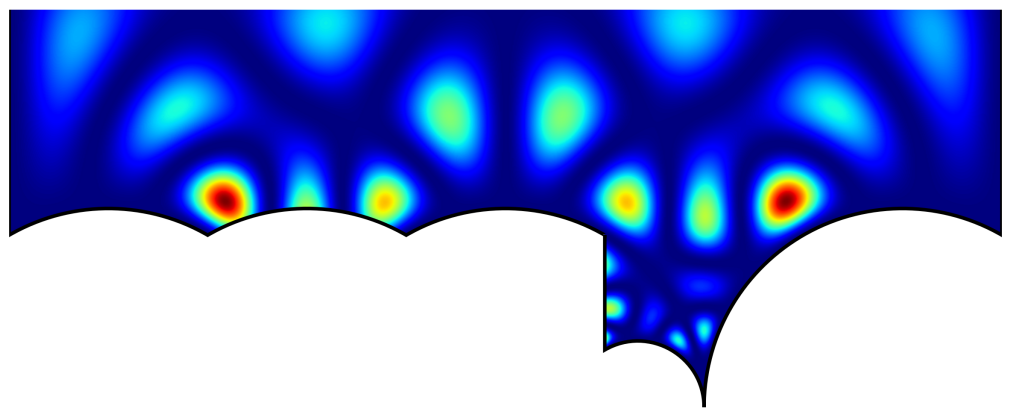

(a) $R=9.67736151045037$

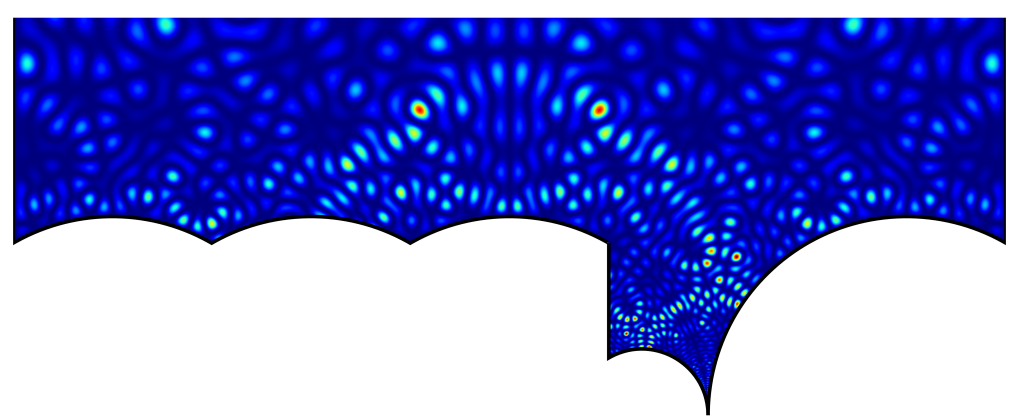

(b) $R=50.1682919704053$

Figure 2: Density plots of Maass cusp forms for $\Gamma(7 ; 0,2,1,1 ; 10)$

\subsection{Residual eigenvalues}

Recall that the residual spectrum consists of eigenvalues $\lambda=s(1-s)$ where $s$ is a pole of the scattering determinant in the interval $(1 / 2,1]$. It follows from the functional equation $\varphi(s) \varphi(1-s)=1$ that $s$ is a pole of $\varphi(s)$ if and only if $1-s$ is a zero and it is therefore sufficient to locate zeros in the interval $[0,1 / 2)$. To find the zeros of $\varphi(s)$ in a rectangle $R$ we first use the argument principle and a recursive subdivision to obtain a list of rectangles containing precisely one zero each. Then, if $R$ contains a single zero, say $z^{*}$, this is located using the general argument principle:

$$
z^{*}=\oint_{\partial R} z \frac{\varphi^{\prime}(z)}{\varphi(z)} d z=z_{0}-\frac{1}{2 \pi i} \oint_{\partial R} \log \varphi(z) d z,
$$

where $z_{0}$ is a specific point on the rectangle, for instance the lower left corner, and the integral over the closed boundary is evaluated starting at $z_{0}$ and Log is using a continuous choice of branch of the argument. In our specific implementation we use a standard Gauss quadrature to evaluate the final line integral. 


\begin{tabular}{clcc} 
Signature & \multicolumn{1}{c}{$\sigma_{T}$} & Generalized level & $\sigma$ \\
\hline$(13 ; 0,3,1,1)$ & $(1324695)(78101211)(13)$ & 35 & 0.52849516 \\
$(14 ; 0,2,2,2)$ & $(12711148)(36591213104)$ & 24 & 0.54590140 \\
$(14 ; 0,2,2,2)$ & $(143711148)(2659121310)$ & 7 & 0.56128255 \\
$(14 ; 0,3,0,2)$ & $(143571062)(89111312)(14)$ & 40 & 0.58050945 \\
$(14 ; 0,3,0,2)$ & $(1582)(39124)(6713101114)$ & 12 & 0.59896234 \\
$(15 ; 0,3,3,0)$ & $(147)(2856910131411)(31215)$ & 9 & 0.53902911 \\
$(15 ; 0,3,3,0)$ & $(1243681175)(910121413)(15)$ & 45 & 0.62465859 \\
$(15 ; 0,3,3,0)$ & $(12410135)(36147811129)(15)$ & 24 & 0.62522158 \\
$(15 ; 0,3,3,0)$ & $(12410135)(36141112159)(78)$ & 42 & 0.61939437 \\
$(15 ; 0,3,3,0)$ & $(12410135)(369)(7814111215)$ & 6 & 0.65696249 \\
$(15 ; 0,2,1,3)$ & $(132812159)(476101314115)$ & 56 & 0.61388854 \\
$(15 ; 0,2,5,0)$ & $(1261013141157)(34812159)$ & 18 & 0.59840142 \\
$(15 ; 0,4,1,0)$ & $(12681175)(34)(910121413)(15)$ & 70 & 0.64139101 \\
$(15 ; 0,4,1,0)$ & $(126348115)(7)(910121413)(15)$ & 40 & 0.64618923 \\
$(15 ; 0,4,1,0)$ & $(125)(3468117)(910121413)(15)$ & 30 & 0.67281282
\end{tabular}

Table 7: Poles $s=\sigma$ of $\varphi(s)$ with $1 / 2<\sigma<1$, all with an error estimate of $2 \cdot 10^{-8}$

For the modular group the explicit formula for $\varphi(s)$ shows that the residual spectrum is trivial and only contains $\lambda=0$, corresponding to $s=1$, and the same is true for some other families congruence subgroups, like $\Gamma_{0}(N)$.

Using the method described above we calculated the residual spectrum for all subgroups of index at most 17 and found non-trivial eigenvalues for 59 of the subgroups with index between 13 and 17 and for each of these we found precisely one residual eigenvalue. Table 7 contains the computed data for indices up to 15 . all eigenvalues are estimated to be correct up to an error of magnitude $2 \cdot 10^{-8}$.

\subsection{On completeness of lists of eigenvalues}

To illustrate the process of using the average Weyl's law to determine whether or not a list of eigenvalues is complete we consider the example of $\Gamma(7 ; 0,2,1,1 ; 10)$ and the list of eigenvalues in Table 3 together with the eigenvalue zero. With notation as before

$$
E(T)=N_{\exp }(T)+M_{\exp }(T)-\frac{7}{12} T^{2}+\frac{2}{\pi} T \log (2 T / e)-\frac{115}{144},
$$

where we used the calculated value of $\Phi(s))=-\mathbb{1}$ to evaluate the constant $c_{G}$.

It is possible to again use the algorithms from Section 8.7 to evaluate $\varphi(1 / 2+i t)$ with an error of about $1 \cdot 10^{-13}$ in $150 \mathrm{~ms}$ at $t=10$ and $350 \mathrm{~ms}$ at $t=20$ using a single core on a standard Intel Xeon E5-2660 CPU.

While this might seem entirely sufficient to compute the function $M(T)$ to a reasonable precision it turns out that the the presence of poles of $\varphi(s)$ close to the line $\Re(s)=1 / 2$ causes the argument of $\varphi(1 / 2+i t)$ to change very rapidly. As an example consider Figure 3 (a) - (d). Subplot (a) shows $E(T)$ where $M(T)$ is computed using a fixed interval size of $10^{-5}$ and it clearly shows that there is either an eigenvalue missing or we have missed an entire turn of $M(T)$ at some point around $T=8$. A magnification of the relevant part of the graph (c) reveals a "bump" of magnitude 0.25 close to $T=7.84$. A renewed calculation with adaptive step size, illustrated in (d), shows that there is indeed a missing complete turn here with $M(T)$ decreasing at a rate of about 828. An independent search for zeros of $\varphi(s)$ revealed a zero of $\varphi(s)$ close to the half-line 


\begin{tabular}{cl}
\multicolumn{1}{c}{$\Gamma(7 ; 0,2,1,1 ; 10)$} & \\
$s$ & Error \\
\hline $0.572733065+1.976751925 i$ & $5 \cdot 10^{-10}$ \\
$0.733513575+3.375697229 i$ & $3 \cdot 10^{-9}$ \\
$0.534077067+4.033089787 i$ & $5 \cdot 10^{-9}$ \\
$0.636831204+4.647281035 i$ & $3 \cdot 10^{-9}$ \\
$0.578894318+5.218542906 i$ & $5 \cdot 10^{-9}$ \\
$0.576128364+5.740065201 i$ & $5 \cdot 10^{-10}$ \\
$0.712936665+5.931656436 i$ & $3 \cdot 10^{-9}$ \\
$0.511603498+6.373898733 i$ & $6 \cdot 10^{-9}$ \\
$0.529113447+6.993308974 i$ & $5 \cdot 10^{-10}$ \\
$0.500123176+7.843821725 i$ & $3 \cdot 10^{-8}$ \\
$0.804713953+8.021994888 i$ & $4 \cdot 10^{-9}$ \\
$0.508112457+8.087176693 i$ & $2 \cdot 10^{-9}$ \\
$0.578811417+8.583881045 i$ & $2 \cdot 10^{-9}$ \\
$0.507979773+8.887458090 i$ & $9 \cdot 10^{-10}$ \\
$0.597375474+9.064110991 i$ & $2 \cdot 10^{-8}$ \\
$0.501820568+9.279816666 i$ & $2 \cdot 10^{-9}$ \\
$0.518175331+9.532449467 i$ & $4 \cdot 10^{-9}$ \\
$0.743162005+9.734794680 i$ & $5 \cdot 10^{-9}$ \\
$0.529459612+9.897753902 i$ & $7 \cdot 10^{-9}$
\end{tabular}

\begin{tabular}{cl}
\multicolumn{1}{c}{$\Gamma(7 ; 0,2,1,1 ; 6)$} & \\
$s$ & Error \\
\hline $0.967441570+1.735920495 i$ & $2 \cdot 10^{-9}$ \\
$0.550661283+2.448560792 i$ & $3 \cdot 10^{-9}$ \\
$0.613351172+3.353051161 i$ & $3 \cdot 10^{-9}$ \\
$0.841054922+3.590272898 i$ & $7 \cdot 10^{-9}$ \\
$0.535600005+3.957558007 i$ & $2 \cdot 10^{-9}$ \\
$0.507905955+4.750759633 i$ & $2 \cdot 10^{-9}$ \\
$0.538548210+5.026498170 i$ & $2 \cdot 10^{-9}$ \\
$0.785442907+5.133366299 i$ & $2 \cdot 10^{-9}$ \\
$0.549270364+5.389216334 i$ & $2 \cdot 10^{-9}$ \\
$0.548870559+5.701826617 i$ & $8 \cdot 10^{-9}$ \\
$0.508207069+5.945138320 i$ & $9 \cdot 10^{-10}$ \\
$0.551735033+6.086341159 i$ & $4 \cdot 10^{-9}$ \\
$0.501006626+6.385351857 i$ & $5 \cdot 10^{-10}$ \\
$0.779918148+6.627319594 i$ & $3 \cdot 10^{-9}$ \\
$0.504894510+6.741907670 i$ & $2 \cdot 10^{-9}$ \\
$0.654810433+6.934126508 i$ & $4 \cdot 10^{-9}$ \\
$0.541560608+7.042933521 i$ & $4 \cdot 10^{-9}$ \\
$0.749999984+7.067362595 i$ & $2 \cdot 10^{-9}$ \\
$0.504757748+7.321269688 i$ & $2 \cdot 10^{-10}$
\end{tabular}

Table 8: Zeros of $\varphi(s)$ with $1 / 2<\Re(s)<1$

at $s=0.50012317+7.84382174 i$. See Table 8 for a complete list up to height $\Im(s)=10$. For a plot of $E(T)$ and $\langle E(T)\rangle$ over a slightly larger range of $T$ see Figure $4(\mathrm{~b})$.

In contrast, consider the group $\Gamma(7 ; 0,2,1,1 ; 6)$ where we have found no new discrete eigenvalues. To justify our belief that there are indeed no such eigenvalues we again study the average Weyl's law, which again turn out to have the form (23). The constants are the same but the counting functions and winding number are of course different. In this case the plot of $E(T)$ and $\langle E(T)\rangle$ in Figure $4(\mathrm{a})$ is very similar to that of $\Gamma(7 ; 0,2,1,1 ; 10)$ even though we have much fewer discrete eigenvalues (only old forms from $\mathrm{PSL}_{2}(\mathbb{Z})$ ). When counting the eigenvalues with $R \leq 10$ together with the zeros of $\varphi(s)$ in the strip $1 / 2<\Re(s)<1$ and imaginary part up to 10 then we find $17+19=36$ for $\Gamma(7 ; 0,2,1,1 ; 10)$ and $6+36=42$ for $\Gamma(7 ; 0,2,1,1 ; 10)$. See also Table 8 which contains all the zeros we have found in this region for $\Gamma(7 ; 0,2,1,1 ; 10)$ (and a subset for $\Gamma(7 ; 0,2,1,1 ; 10)$ ).

\subsection{Fourier coefficients}

For congruence subgroups the generalized Ramanujan-Petersson conjecture implies that the Fourier coefficients of a Maass cusp form should satisfy a bound of the form $|c(p)| \leq 2$ when $p$ is a prime. It is also expected that the coefficients $c(p)$ behave like independent random variables, distributed according to a Sato-Tate (semi-circle) distribution. See e.g. [40] and also experimental data in [63, 65]. For "generic" subgroups, in particular nonarithmetic groups, on the other hand, the Fourier coefficients are expected to behave like Gaussian distributed random variables. Since this question has not been previously investigated for noncongruence subgroups we computed a larger number of Fourier coefficients for a few select examples.

In the definition of a newform we assume that the first non-zero Fourier coefficient at infinity is normalized to 1 . In all examples of newforms that we have computed it has 


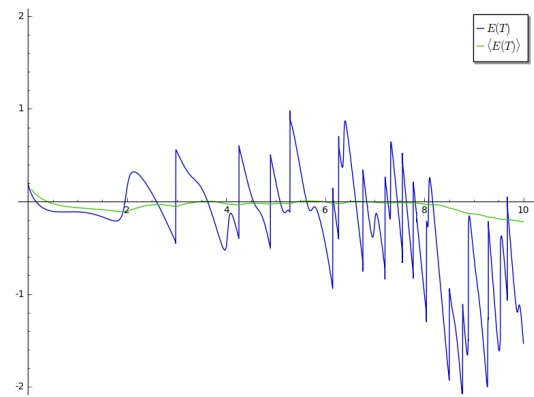

(a) Fixed interval size 0.0005

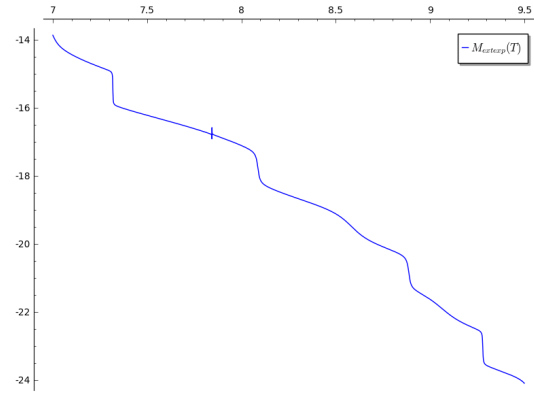

(c) Fixed interval size 0.0005

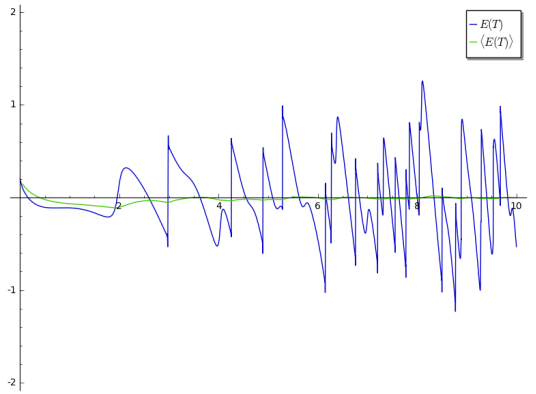

(b) Aptive interval size $>7 \cdot 10^{-7}$

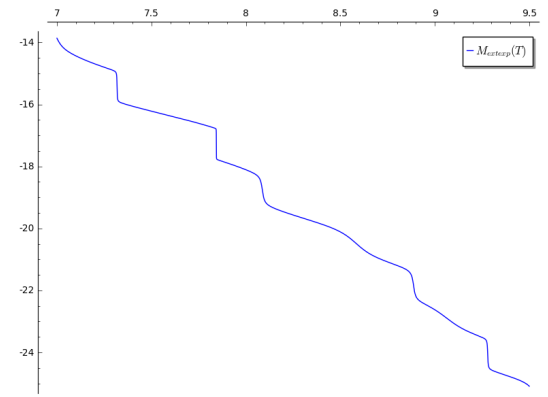

(d) Adaptive interval size $>7 \cdot 10^{-7}$

Figure 3: Error function $E(t)$, average $\langle E(t)\rangle$ and winding number $M_{\exp }(T)$ for $\Gamma(7 ; 0,2,1,1 ; 10)$

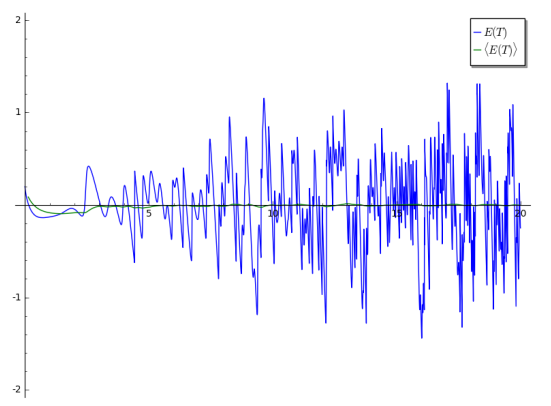

(a) $\Gamma(7 ; 0,2,1,1 ; 6)$

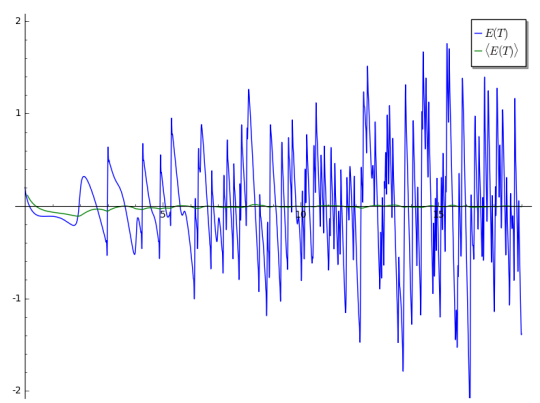

(b) $\Gamma(7 ; 0,2,1,1 ; 10)$

Figure 4: Error function $E(T)$ and average $\langle E(T)\rangle$ 


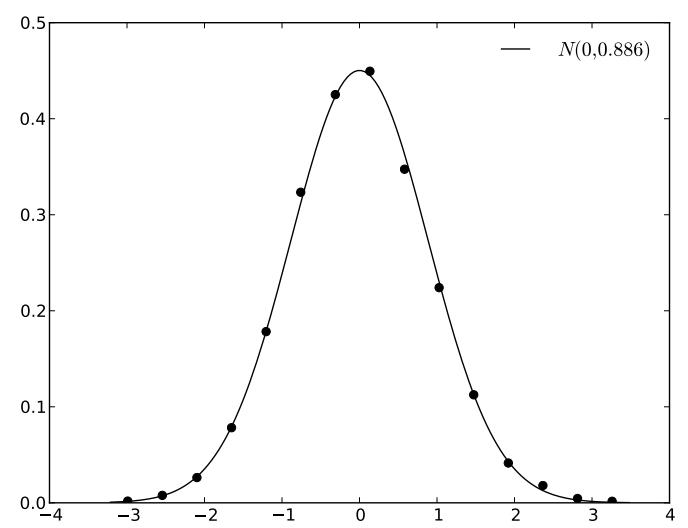

Figure 5: Gaussian fit of 12422 coefficients for $\Gamma(7 ; 0,2,1,1 ; 12)$ and $R=3.341 \ldots$

been possible to set $c_{1}(1)=1$. Only in the case of oldforms has it been necessary to use other coefficients.

Figure 5 shows the value-distribution of 12422 (real) Fourier coefficients of a newform with eigenvalue $R=3.34168098977$ on $\Gamma(7 ; 0,2,1,1 ; 12)$ compared to a normal (Gaussian) distribution with mean 0 and standard deviation 0.886 , as given by the coefficients.

As a second example we studied the Fourier coefficients of a newform on $G=$ $\Gamma(10 ; 0,2,2,1 ; 8,1)$ with eigenvalue $R=2.40931877484576$. The symmetry in this case is given by $\alpha: z \mapsto 1-\bar{z}$ and the cusp width at infinity is 8 . Hence the Fourier coefficients will not be real in this case, but have arguments shifted by multiples $\frac{\pi}{8}$, to be precise

$$
c(n)=a(n) e^{\frac{\pi i}{8}(1-n)} \quad \text { with } \quad a(n) \in \mathbb{R},
$$

as shown in Section 6.3. The surprising observation here is that it seems that the different residue classes modulo 8 also have different standard deviation, as can be seen in figure 6 (a)-(e), which shows the distribution of the real-valued coefficients $a(n)$ separated into congruence classes with respect to $n \bmod 8$ and compared to the normal distribution with mean 0 and standard deviation given by the $a(n) \mathrm{s}$. In all of these cases the fit to the Gaussian density looks good and it seems plausible that the coefficients are indeed similar to random variables sampled from a normal distribution.

\subsection{Exceptional eigenvalues}

The final topic for our investigation of spectra for noncongruence subgroups is exceptional eigenvalues, that is, small eigenvalues which are associated with Maass cusp forms. For more details see Section 3.2. By Zograf's theorem [73] we know that a cycloidal group of index 96 and genus 0 must have exceptional eigenvalues. By using the algorithms described earlier we found examples of such groups for 5 different signatures and at least one, sometimes two, exceptional eigenvalues on each of these groups.

We used a number of heuristic tests to confirming that these eigenvalues indeed correspond to Maass cusp forms, including checking that the absolute value of $c(-1)$ is 


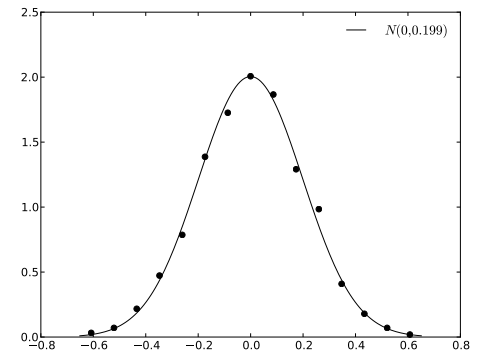

(a) Coefficients $a(n)$ with $n \equiv 0 \bmod 8$

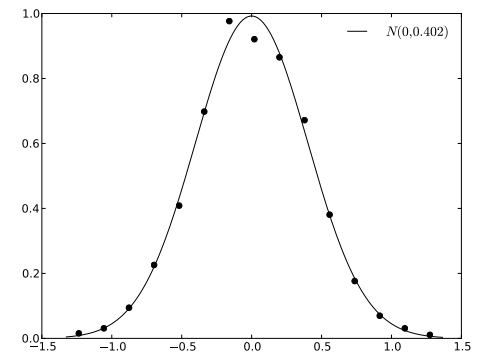

(c) Coefficients $a(n)$ with $n \equiv \pm 2 \bmod 8$

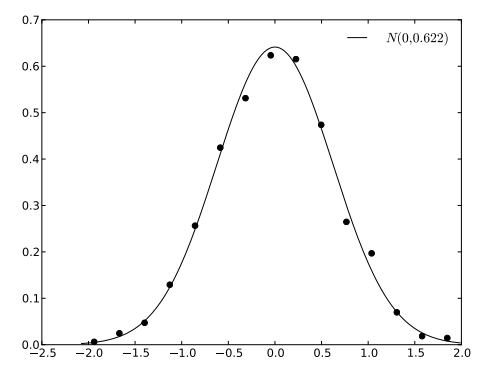

(e) Coefficients $a(n)$ with $n \equiv 0 \bmod 8$

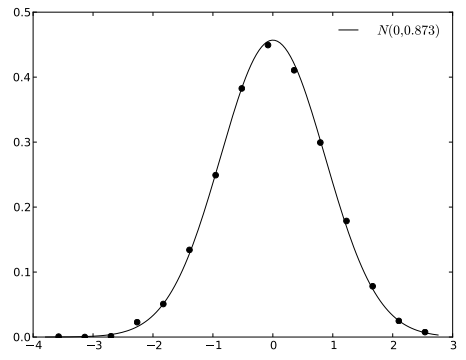

(b) Coefficients $a(n)$ with $n \equiv \pm 1 \bmod 8$

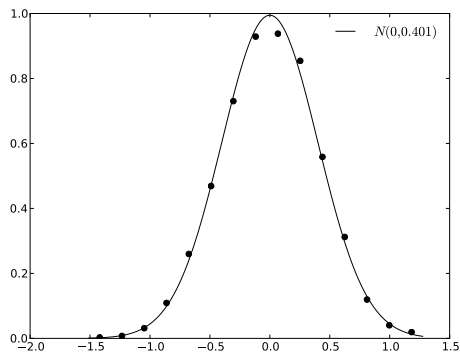

(d) Coefficients $a(n)$ with $n \equiv 4 \bmod 8$

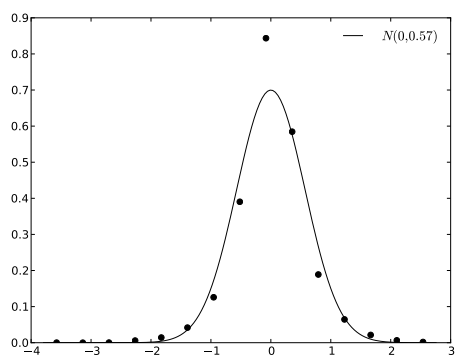

(f) All coefficients

Figure 6: Gaussian fit of Fourier coefficients for $\Gamma(10 ; 0,2,2,1 ; 8 ; 1)$ and $R=2.049 \ldots$ 
Figure 7: A density plot of a Maass cusp form with exceptional eigenvalue on a group of index 48

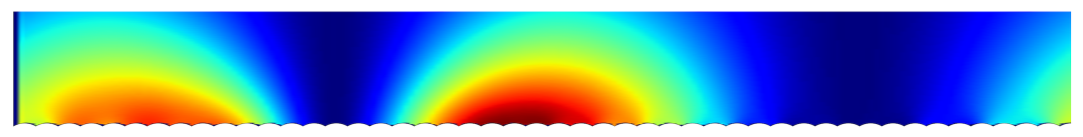

close to 1 . Since none of these groups have a reflectional symmetry we do not expect $c(-1)$ to be a root of unity. This was confirmed by the computations, as can be seen in Table 9 , which contains all the exceptional eigenvalues we found for these groups. Note that in these tables we decided to list $\lambda=1 / 4+R^{2}$ itself rather than the parameter $R$.

If we believe in Selberg's conjecture then there should not be any exceptional eigenvalues for congruence subgroups and indeed, we did not find any exceptional eigenvalues for any of the subgroups up to index 18 which we classified. Since the list of subgroups between index 96 and 18 is rather long we "randomly" checked groups amenable to computation, e.g cycloidal groups of genus 0 of various indices between 18 and 96 .

The only other index for which we found exceptional eigenvalues in this search was index 48 where we found 3 such groups. See Table 10 and also Figure 7 . The existence of these exceptional eigenvalues indicate that Zograf's theorem, and in particular the bound he give for the first eigenvalue: $\lambda_{1}<8 \pi(g+1) /\left(\mathcal{F}_{G}\right)$, is not sharp. It would be very interesting to see if it is possible to improve Zograf's theorem in a way which can explain also these eigenvalues or if they are beyond a purely geometric approach.

\begin{tabular}{lcrcc}
\hline Signature & $\lambda=1 / 4+R^{2}$ & $c(-1)$ & ||$c(-1)|-1|$ \\
\hline$(96,0,1,2,24)$ & 0.18243512022064 & $-0.290561475101079-0.956856325947566 i$ & $3 \cdot 10^{-10}$ \\
$(96,0,1,2,24)$ & 0.08728303723840 & $0.290325721699923+0.956927884053285 i$ & $2 \cdot 10^{-11}$ \\
$(96,0,1,6,21)$ & 0.18084119616407 & $-0.433509032349625-0.901149221155274 i$ & $4 \cdot 10^{-11}$ \\
$(96,0,1,10,18)$ & 0.17866865406717 & $-0.576297458202358-0.817240013498072 i$ & $4 \cdot 10^{-12}$ \\
$(96,0,1,10,18)$ & 0.08637835017746 & $0.540882479332576+0.841098177126570 i$ & $7 \cdot 10^{-12}$ \\
$(96,0,1,14,15)$ & 0.18039028688469 & $-0.674042684512656-0.738692398176520 i$ & $2 \cdot 10^{-10}$ \\
$(96,0,1,14,15)$ & 0.08499600279378 & $0.628289200748876+0.777979871324950 i$ & $2 \cdot 10^{-11}$ \\
$(96,0,1,18,12)$ & 0.18378606746903 & $-0.719011587442541-0.694998084138351 i$ & $8 \cdot 10^{-11}$ \\
$(96,0,1,18,12)$ & 0.08352690935761 & $0.670044016537574+0.742321369702485 i$ & $7 \cdot 10^{-12}$ \\
\hline
\end{tabular}

Table 9: Small Eigenvalues on groups of index 96 and genus 0

\begin{tabular}{lccc}
\hline Signature & $\lambda=1 / 4+R^{2}$ & $c(-1)$ & ||$c(-1)|-1|$ \\
\hline$(48,0,1,2,12)$ & 0.19958333500894 & $0.557382098772570+0.830256102643485 i$ & $4 \cdot 10^{-12}$ \\
$(48,0,1,6,9)$ & 0.19551437034185 & $0.761233264784584+0.648478154295867 i$ & $7 \cdot 10^{-12}$ \\
$(48,0,1,10,6)$ & 0.18842480680558 & $0.823802234041514+0.566877305246636 i$ & $8 \cdot 10^{-12}$ \\
\hline
\end{tabular}

Table 10: Small Eigenvalues on groups of index 48 and genus 0

\section{Acknowledgments}

Many of the ideas and algorithms in this paper originates in my Ph.D. thesis and I am grateful to my thesis advisor Dennis Hejhal and my second advisor Andreas Strömbergs- 
son for many helpful discussions and comments. I am also grateful for the anonymous referee, who's careful comments on a previous version were very useful and inspired me to add several sections.

\section{References}

[1] A. O. L. Atkin, J. Lehner, Hecke operators on $\Gamma_{0}(M)$, Math. Ann. 185 (1970) 134-160.

[2] A. O. L. Atkin, W.-C. W. Li, L. Long, On Atkin and Swinnerton-Dyer congruence relations. II, Math. Ann. 340 (2) (2008) 335-358.

[3] A. O. L. Atkin, H. P. F. Swinnerton-Dyer, Modular forms on noncongruence subgroups, in: Combinatorics (Proc. Sympos. Pure Math., Vol. XIX, Univ. California, Los Angeles, Calif., 1968), Amer. Math. Soc., Providence, R.I., 1971, pp. 1-25.

[4] H. Avelin, Deformation of $\Gamma_{0}(5)$-cusp forms, Math. Comp. 76 (257) (2007) 361-384 (electronic).

[5] H. Avelin, Computations of Eisenstein series on Fuchsian groups, Math. Comp. 77 (263) (2008) 1779-1800. doi:10.1090/S0025-5718-08-02092-9. URL https://doi.org/10.1090/ S0025-5718-08-02092-9

[6] H. Avelin, Computations of Green's function and its Fourier coefficients on Fuchsian groups, Experiment. Math. 19 (3) (2010) 317-334. doi:10.1080/10586458.2010.10390626. URL https: //doi.org/10.1080/10586458.2010.10390626

[7] G. Berger, Hecke operators on noncongruence subgroups, C. R. Acad. Sci. Paris Sér. I Math. 319 (9) (1994) 915-919.

[8] V. Blomer, F. Brumley, The role of the Ramanujan conjecture in analytic number theory, Bull. Amer. Math. Soc. (N.S.) 50 (2) (2013) 267-320. doi:10.1090/S0273-0979-2013-01404-6.

[9] A. Booker, A. Strömbergsson, Numerical computations with the trace formula and the Selberg eigenvalue conjecture, J. Reine Angew. Math. 607 (2007) 113-161.

[10] A. R. Booker, D. J. Platt, Turing's method for the selberg zeta-function, arXiv:1710.00603 (December 2017).

[11] A. R. Booker, A. Strömbergsson, A. Venkatesh, Effective computation of Maass cusp forms, Int. Math. Res. Not. (2006) Art. ID 71281, 34doi:10.1155/IMRN/2006/71281. URL http://dx.doi.org/ 10.1155/IMRN/2006/71281

[12] J. H. Bruinier, F. Strömberg, Computation of harmonic weak Maassforms, Exp. Math. 21 (2) (2012) $117-131$.

[13] C. J. Cummins, S. Pauli, Congruence subgroups of $\operatorname{PSL}(2, \mathbb{Z})$ of genus less than or equal to 24 Experiment. Math. 12 (2) (2003) 243-255. URL http://projecteuclid.org/getRecord?id=euclid. em/1067634734

[14] A. Erdélyi, W. Magnus, F. Oberhettinger, F. G. Tricomi, Higher Transcendental Functions. Vols. I, II, McGraw-Hill, 1953.

[15] L. Fang, J. W. Hoffman, B. Linowitz, A. Rupinski, H. Verrill, Modular forms on noncongruence subgroups and Atkin-Swinnerton-Dyer relations, Experiment. Math. 19 (1) (2010) 1-27. URL http: //projecteuclid.org/getRecord?id=euclid.em/1268404801

[16] D. W. Farmer, S. Lemurell, Deformations of Maass forms, Math. Comp. 74 (252) (2005) 1967-1982.

[17] C. Franc, G. Mason, Hypergeometric series, modular linear differential equations and vector-valued modular forms, Ramanujan J. 41 (1-3) (2016) 233-267. doi:10.1007/s11139-014-9644-x.

[18] The GAP Group, GAP - Groups, Algorithms, and Programming, Version 4.8 .8 (2017).

[19] E. Girondo, J. Wolfart, Conjugators of Fuchsian groups and quasiplatonic surfaces, Q. J. Math. 56 (4) (2005) 525-540. doi:10.1093/qmath/hah054. URL https://doi.org/10.1093/qmath/hah054

[20] C. Godsil, W. Imrich, R. Razen, On the number of subgroups of given index in the modular group, Monatsh. Math. 87 (4) (1979) 273-280. doi:10.1007/BF01637030.

[21] D. A. Hejhal, The Selberg trace formula for $\operatorname{PSL}(2, \mathbf{R})$. Vol. 2, Vol. 1001 of Lecture Notes in Mathematics, Springer-Verlag, Berlin, 1983.

[22] D. A. Hejhal, Eigenvalues of the Laplacian for Hecke triangle groups, Mem. Amer. Math. Soc. 97 (469) (1992) vi +165 .

[23] D. A. Hejhal, On the calculation of Maass cusp forms, in: Hyperbolic geometry and applications in quantum chaos and cosmology, Vol. 397 of London Math. Soc. Lecture Note Ser., Cambridge Univ. Press, Cambridge, 2012, pp. 175-185.

[24] T. Hsu, Identifying congruence subgroups of the modular group, Proc. Amer. Math. Soc. 124 (5) (1996) 1351-1359. 
[25] M. N. Huxley, Scattering matrices for congruence subgroups, in: R. Rankin (Ed.), Modular forms, Ellis Horwood, Chichester, 1984, pp. 141-156.

[26] M. N. Huxley, Exceptional eigenvalues and congruence subgroups, in: The Selberg trace formula and related topics (Brunswick, Maine, 1984), Vol. 53 of Contemp. Math., Amer. Math. Soc., Providence, RI, 1986, pp. 341-349. doi:10.1090/conm/053/853564.

[27] F. M. Ives, Permutation enumeration: four new permutation algorithms, Commun. ACM 19 (2) (1976) 68-72. doi:http://doi.acm.org/10.1145/359997.360002.

[28] H. Iwaniec, Spectral methods of automorphic forms, 2nd Edition, Vol. 53 of Graduate Studies in Mathematics, American Mathematical Society, Providence, RI, 2002.

[29] J. Jorgenson, L. Smajlović, H. Then, On the distribution of eigenvalues of Maass forms on certain moonshine groups, Math. Comp. 83 (290) (2014) 3039-3070. URL https://doi.org/10.1090/ S0025-5718-2014-02823-8

[30] H. H. Kim, Functoriality for the exterior square of $\mathrm{GL}_{4}$ and the symmetric fourth of $\mathrm{GL}_{2}$, J. Amer. Math. Soc. 16 (1) (2003) 139-183, with appendix 1 by Dinakar Ramakrishnan and appendix 2 by Kim and Peter Sarnak. doi:10.1090/S0894-0347-02-00410-1.

[31] I. Kiming, M. Schütt, H. A. Verrill, Lifts of projective congruence groups, J. Lond. Math. Soc. (2) 83 (1) (2011) 96-120. doi:10.1112/jlms/jdq062.

[32] R. S. Kulkarni, An arithmetic-geometric method in the study of the subgroups of the modular group, Amer. J. Math. 113 (6) (1991) 1053-1133. doi:10.2307/2374900.

[33] C. Kurth, Modular forms and modular symbols for noncongruence groups, ProQuest LLC, Ann Arbor, MI, 2009, thesis (Ph.D.)-Iowa State University.

[34] C. Kurth, L. Long, On modular forms for some noncongruence subgroups of $\mathrm{SL}_{2}(\mathbb{Z})$, J. Number Theory 128 (7) (2008) 1989-2009.

[35] C. Kurth, L. Long, On modular forms for some noncongruence subgroups of $\mathrm{SL}_{2}(\mathbb{Z})$. II, Bull. Lond. Math. Soc. 41 (4) (2009) 589-598. doi:10.1112/blms/bdp061.

[36] C. A. Kurth, L. Long, Computations with finite index subgroups of $\mathrm{PSL}_{2}(\mathbb{Z})$ using Farey symbols, in: Advances in algebra and combinatorics, World Sci. Publ., Hackensack, NJ, 2008, pp. 225-242.

[37] W.-C. W. Li, L. Long, Fourier coefficients of noncongruence cuspforms, Bull. Lond. Math. Soc. 44 (3) (2012) 591-598. doi:10.1112/blms/bdr122.

[38] W.-C. W. Li, L. Long, Z. Yang, On Atkin-Swinnerton-Dyer congruence relations, J. Number Theory 113 (1) (2005) 117-148.

[39] L. Long, On Atkin and Swinnerton-Dyer congruence relations. III, J. Number Theory 128 (8) (2008) 2413-2429. doi:10.1016/j.jnt.2008.02.014.

[40] W. Luo, F. Zhou, On the hecke eigenvalues of maass forms, accepted (2017).

[41] C. Marks, Fourier coefficients of three-dimensional vector-valued modular forms, Commun. Number Theory Phys. 9 (2) (2015) 387-412. doi:10.4310/CNTP.2015.v9.n2.a5.

[42] M. H. Millington, On cycloidal subgroups of the modular group, Proc. London Math. Soc. (3) 19 (1969) $164-176$.

[43] M. H. Millington, Subgroups of the classical modular group, J. London Math. Soc. (2) 1 (1969) 351-357.

[44] T. Miyake, On automorphic forms on $\mathrm{GL}_{2}$ and Hecke operators, Ann. of Math. (2) 94 (1971) 174-189.

[45] H. Monien, Modular forms of non-congruence subgroups of $\operatorname{PSL}(2, \mathbb{Z})$, preprint (2013).

[46] R. Phillips, P. Sarnak, Cusp forms for character varieties, Geom. Funct. Anal. 4 (1) (1994) 93-118.

[47] R. S. Phillips, P. Sarnak, On cusp forms for co-finite subgroups of PSL(2, R), Invent. Math. 80 (2) (1985) 339-364.

[48] R. S. Phillips, P. Sarnak, The Weyl Theorem and the Deformation of Discrete Groups, Comm. on Pure and Appl. Math. 38 (1985) 853-866.

[49] H. Rademacher, H. S. Zuckerman, On the Fourier coefficients of certain modular forms of positive dimension, Ann. of Math. (2) 39 (2) (1938) 433-462. doi:10.2307/1968796.

[50] M. Richards, Computing covering maps for subgroups of $\Gamma(1)$ with genus $\geq 1$., Ph.D. thesis, University of Oxford (1995).

[51] M. S. Risager, Asymptotic densities of Maass newforms, J. Number Theory 109 (1) (2004) 96-119.

[52] M. S. Risager, On Selberg's small eigenvalue conjecture and residual eigenvalues, J. Reine Angew. Math. 656 (2011) 179-211. doi:10.1515/CRELLE.2011.050.

[53] P. Sarnak, On cusp forms, in: The Selberg trace formula and related topics (Brunswick, Maine, 1984), Vol. 53 of Contemp. Math., Amer. Math. Soc., Providence, RI, 1986, pp. 393-407. doi: $10.1090 /$ conm/053/853570.

[54] P. Sarnak, Selberg's eigenvalue conjecture, Notices Amer. Math. Soc. 42 (11). 
[55] A. J. Scholl, On the Hecke algebra of a noncongruence subgroup, Bull. London Math. Soc. 29 (4) (1997) 395-399. doi:10.1112/S0024609396002639.

[56] A. Selberg, On the estimation of Fourier coefficients of modular forms, in: Proc. Sympos. Pure Math., Vol. VIII, Amer. Math. Soc., Providence, R.I., 1965, pp. 1-15.

[57] A. Selberg, Collected Papers, vol. 1, Springer-Verlag, 1989.

[58] W. Stein, Modular forms, a computational approach, Vol. 79 of Graduate Studies in Mathematics, American Mathematical Society, Providence, RI, 2007, with an appendix by Paul E. Gunnells

[59] W. A. Stein, et. al., Sage Mathematics Software (Version 8.0), The Sage Development Team (2017). URL http://www. sagemath.org

[60] W. A. Stein, F. Strömberg, S. Ehlen, N. Skoruppa, et. al., Algorithms for automorphic forms and related objects in psage (2017). URL http://github.com/fredstro/psage

[61] T. J. Stieltjes, Sur le développement de $\log \gamma(a)$, Journal de mathématiques pures et appliquées 4e série 4 (1889) 425-466.

[62] A. Strohmaier, V. Uski, Eigenvalues and spectral determinants on compact hyperbolic surfaces, in: Microlocal methods in mathematical physics and global analysis, Trends Math., Birkhäuser/Springer, Basel, 2013, pp. 105-107. URL https://doi.org/10.1007/ 978-3-0348-0466-0_24

[63] F. Strömberg, Computational aspects of maass waveforms, Ph.D. thesis, Uppsala University (2005)

[64] F. Strömberg, Computation of Maass waveforms with nontrivial multiplier systems, Math. Comp. 77 (264) (2008) 2375-2416. doi:10.1090/S0025-5718-08-02129-7. URL https://doi.org/10. 1090/S0025-5718-08-02129-7

[65] F. Strömberg, Maass waveforms on $\left(\Gamma_{0}(N), \chi\right)$ (computational aspects), in: Hyperbolic geometry and applications in quantum chaos and cosmology, Vol. 397 of London Math. Soc. Lecture Note Ser., Cambridge Univ. Press, Cambridge, 2012, pp. 187-228.

[66] F. Strömberg, Newforms and spectral multiplicity for $\Gamma_{0}(9)$, Proc. Lond. Math. Soc. (3) 105 (2) (2012) 281-310. doi:10.1112/plms/pds004. URL https://doi.org/10.1112/plms/pds004

[67] F. Strömberg, Examples, code and data for "noncongruence subgroups and maass waveforms" (2018). URL https://github.com/fredstro/noncongruence

[68] A. Strömbergsson, Studies in the analytical and spectral theory of automorphic forms, Ph.D. thesis, Uppsala University, Dept. of Math. (2001).

[69] H. Then, Maass cusp forms for large eigenvalues, Math. Comp. 74 (249) (2005) 363-381.

[70] A. B. Venkov, A remark on the discrete spectrum of the automorphic Laplacian for a generalized cycloidal subgroup of a general Fuchsian group, J. Soviet Math. 52 (3) (1990) 3016-3021.

[71] A. B. Venkov, Spectral Theory of Automorphic Functions and Its Applications, Kluwer Academic Publishers, 1990

[72] K. Wohlfahrt, An extension of F. Klein's level concept, Illinois J. Math. 8 (1964) 529-535.

[73] P. G. Zograf, The spectrum of automorphic Laplacians in spaces of parabolic functions, Dokl. Akad. Nauk SSSR 269 (4) (1983) 802-805. 